Acta Numerica (2017), pp. 001-

doi:10.1017/S09624929
(C) Cambridge University Press, 2017

Printed in the United Kingdom

\title{
Algebraic Multigrid Methods
}

\author{
Jinchao Xu and Ludmil Zikatanov*
}

This paper is to give an overview of AMG methods for solving large scale systems of equations such as those from the discretization of partial differential equations. AMG is often understood as the acronym of "Algebraic Multi-Grid", but it can also be understood as "Abstract Muti-Grid". Indeed, as it demonstrates in this paper, how and why an algebraic multigrid method can be better understood in a more abstract level. In the literature, there are a variety of different algebraic multigrid methods that have been developed from different perspectives. In this paper, we try to develop a unified framework and theory that can be used to derive and analyze different algebraic multigrid methods in a coherent manner. Given a smoother $R$ for a matrix $A$, such as Gauss-Seidel or Jacobi, we prove that the optimal coarse space of dimension $n_{c}$ is the span of the eigen-vectors corresponding to the first $n_{c}$ eigen-values of $\bar{R} A$ (with $\bar{R}=R+R^{T}-R^{T} A R$ ). We also prove that this optimal coarse space can be obtained by a constrained trace-minimization problem for a matrix associated with $\bar{R} A$ and demonstrate that coarse spaces of most of existing AMG methods can be viewed some approximate solution of this trace-minimization problem. Furthermore, we provide a general approach to the construction of a quasi-optimal coarse space and we prove that under appropriate assumptions the resulting two-level AMG method for the underlying linear system converges uniformly with respect to the size of the problem, the coefficient variation, and the anisotropy. Our theory applies to most existing multigrid methods, including the standard geometric multigrid method, the classic AMG, energy-minimization AMG, unsmoothed and smoothed aggregation AMG, and spectral AMGe.

\footnotetext{
* Center for Computational Mathematics and Applications, Department of Mathematics, The Pennsylvania State University, University Park, PA 16802, USA.

Email: jinchao@psu.edu \& ludmil@psu.edu
} 


\section{CONTENTS}

1 Introduction

2 Model problems and discretization

2.1 Model elliptic PDE operators

2.2 Examples of finite difference and finite element discretizations

2.3 Spectral properties

2.4 Properties of finite element matrices

3 Linear vector spaces and duals

3.1 Dual and inner product

3.2 Matrix representation

3.3 Eigenvalues and eigenvectors

3.4 Bibliographical notes

4 Basic iterative methods

4.1 Basic iterative methods

4.2 Jacobi and Gauss-Seidel methods

4.3 The method of subspace corrections

4.4 Bibliographical notes

5 Abstract multigrid methods and 2-level theory

5.1 A two-level method

5.2 An optimal two-level AMG theory

5.3 Quasi-optimal theories

5.4 Algebraically high and low frequencies

5.5 Smoothing properties of Jacobi and Gauss-Seidel methods

5.6 Convergence theory in view of algebraic high- and low-frequencies

5.7 Bibliographical notes

6 A general approach to the construction of coarse space 42

6.1 Bibliographical notes

7 Graphs and sparse matrices

7.1 Sparse matrix and its adjacency graph

7.2 $M$-matrix relatives of finite element stiffness matrices

7.3 Bibliographical notes

8 Strength of connections

8.1 Basic idea and strength function

8.2 Classical AMG

8.3 Local-optimization strength function

8.4 Lean AMG

8.5 Bibliographical notes

9 Coarsening strategies

9.1 Motivations

9.2 Basic approach

9.3 Two basic coarsening algorithms

9.4 Adaptive coarsening for classical AMG 
9.5 AGMG coarsening: a pairwise aggregation

9.6 Bibliographical notes

10 GMG, AMG and a geometry-based AMG 69

10.1 Geometric multigrid method

10.2 Obtaining AMG from GMG

10.3 Obtaining GMG from AMG

10.4 Spectral AMGe: a geometry-based AMG

10.5 Bibliographical notes

11 Energy-min AMG

11.1 Energy-minimization versus trace-minimization

11.2 Energy minimization basis for AMG and Schwarz methods

11.3 Convergence of energy minimization AMG

11.4 Bibliographical notes

12 Classical AMG

12.1 Coarse spaces in classical AMG

12.2 Quasi-optimality of ideal interpolation

12.3 Construction of prolongation matrix $P$

12.4 Classical AMG within the abstract AMG framework

12.5 Bibliographical notes

13 Aggregation-based AMG

13.1 Unsmoothed aggregation: preserving 1 kernel vector

13.2 Unsmoothed aggregation: preserving multiple vectors

13.3 Smoothed aggregation

13.4 Bibliographical notes

14 Problems with discontinuous and anisotropic coefficients

14.1 Jump coefficients

14.2 Anisotropic problem

14.3 Bibliographical notes

15 Bootstrap and adaptive AMG

111

15.1 Sparsity of prolongation matrices

15.2 Notation

15.3 A basic adaptive algorithm

15.4 Bibliographical notes

16 Concluding remarks

117

References

118 


\section{Introduction}

Multigrid methods are among the most efficient numerical methods for solving large scale systems of equations, linear and nonlinear alike, arising from the discretization of partial differential equations. This type of methods can be viewed as an acceleration of traditional iterative methods based on local relaxation such as Gauss-Seidel and Jacobi methods. For linear systems arising from finite element or finite difference discretization of elliptic boundary value problems, local relaxation method were observed to converge very fast on the high frequency part of the solution. The low frequency part of the solution, although slow to converge, corresponds to a relatively smoother part of the function that can be well-approximated on a coarser grid. The main idea behind such multigrid methods is to project the error obtained after applying a few iterations of local relaxation methods onto a coarser grid. The projected error equations have two characteristics. Firstly, the resulting system has a smaller size. Secondly, part of the slow-to-converge low frequency error on a finer grid becomes relatively high frequency on the coarser grid and these frequencies can be further corrected by a local relaxation method, but this time on the coarse grid. By repeating such a process and going to further coarser and coarser grids, a multilevel iterative process is obtained. Such algorithms have been proven to have uniform convergence with nearly optimal complexity for a large class of linear algebraic systems arising from the discretization of partial differential equations, especially elliptic boundary problems of 2nd and 4th order. One main component of this type of multilevel algorithm is a hierarchy of geometric grids, typically a sequence of nested grids obtained by successive refinement. The resulting algorithms are known as geometric multigrid (GMG) methods.

Despite of their extraordinary efficiency, however, the GMG methods have their limitations. They depend on a hierarchy of geometric grids which is often not readily available and it can be argued that the range of applicability of the GMG methods is, therefore, limited.

The Algebraic MultiGrid (AMG) methods were designed in an attempt to address such limitations. They were proposed as means to generalize geometric multigrid methods for systems of equations that share properties with discretized PDEs, such as the Laplacian equation, but potentially have unstructured grids in the underlying discretization. The first AMG algorithm in (Brandt, McCormick and Ruge 1982a) was a method developed under the assumption that such a problem was being solved. Later, the AMG algorithm was generalized using many heuristics to extend its applicability to more general problems and matrices. As a result, a variety of AMG methods have been developed in the last three decades and they have been applied to many practical problems with success.

In this paper, we give an overview of AMG methods from a theoretical viewpoint. AMG methods have been developed through a combination of certain theoretical consideration and heuristic arguments, and many AMG methods work, with various degrees of efficiency, for different applications. We find it very hard to give 
a coherent picture on the state of the art of AMG methods if we choose to simply make a comprehensive list of existing algorithms without digging into their theoretical foundation. But, unfortunately, a good theoretical understanding of why and how these methods work is still seriously lacking.

In preparing this article, we have undertaken the task of making a thorough investigation on the design and analysis of AMG from a theoretical point of view. While there are many bits and pieces of ideas spreading out in the literature, we managed to re-examine most of the existing results and "re-invent the wheel" trying to deliver a coherent theoretical description. To do this, we have developed several tools for the design and analysis of AMG.

With very few exceptions, the AMG algorithms have been mostly targeting the solution of symmetric positive definite (SPD) systems. In this paper, we choose to present our studies for a slightly larger class of problems, namely symmetric semi-positive definite (SSPD) systems. This approach is not only more inclusive, but more importantly, the SSPD class of linear systems can be viewed as more intrinsic to the AMG ideas. For example, while the standard discretizations of the Laplacian operator with homogeneous Dirichlet boundary condition results in an SPD system, the design of AMG may be better understood by using local problem (defined on subdomains) with homogeneous Neumann boundary condition, which would amount to an SSPD sub-systems.

In short, in this paper we consider AMG techniques for solving a linear system of equations:

$$
A u=f,
$$

where $A$ is a given SSPD operator or sparse matrix, and the problem is posed in a vector space of a large dimension. The starting point of an AMG procedure is to first choose a smoother, which is often taken to be some local relaxation iterative methods such as the point Jacobi, Gauss-Seidel method, or more generally, overlapping Schwarz methods. The use of pointwise smoothers seems to encompass most of the efforts in the literature in constructing and implementing most (if not all) of the AMG methods. More general smoothers based on overlapping Schwarz methods are necessary for some problems, but we shall not study them in detail in this paper. In any event, any chosen smoother is expected to only converge well on certain components of solution, which will be known as algebraic high frequencies with respect to the given smoother. With the smoother fixed, the main task of an AMG method then is to identify a sequence of coarse spaces that would complement well this smoother. Roughly speaking, an ideal sequence of coarse spaces is such that any vector (namely solution to (1.1) for any $f$ ) in the finest space can be well represented by a linear combination of all the algebraic high frequencies on all coarse spaces. As a result, the AMG method would converge well for the problem (1.1).

It is hard to make a theoretically concise statement for what is said above in the multilevel setting. Instead we will focus on first answering such a question 
for a two-level setting. For a two-level setting, the first theoretical question is as follows: "Given a smoother, say $R$, what is the optimal coarse space of given dimension so that the resulting AMG has the best convergence rate?" This question will be thoroughly addressed in \$5. As it turns out, the optimal coarse space will consist of the eigenvectors corresponding to the lower-end of the spectrum of the a matrix such as $R A$. While our two level theory is theoretically pleasing, it does not offer a practical solution as finding these eigenvectors will be too expensive. Thus, the task of the AMG design is to find good but inexpensive approximation of this algebraic low frequency eigen-space which will still result in an AMG algorithm with desirable convergence properties. We call such an approximation of optimal coarse space as "quasi-optimal" coarse space.

In the design of all these AMG algorithms, one key component is the coarsening of spaces or the graph associated with the matrix $A$. Two main strategies are: independent-set based and the aggregation-based. We will present several approaches on the construction of quasi-optimal coarse spaces. We would especially advocate two approaches that have sound theoretical foundation. The first approach is outlined in $\$ 5$ and later on in $\$ 11$. We will first prove that the optimal coarse space from the theory in $\$ 5$ can be characterized, in a mathematically equivalent manner, by solutions to a trace minimization problem. In a functional setting, such a trace-minimization can be interpreted as minimization of the sum of the energy norm of a set of coarse basis functions. These precise equivalents give a very clear guidance how some AMG methods can be constructed. One practical approach based on such a theorem is to look for energy-minimization basis functions among locally supported function classes. The resulting algorithms are known as energy-min AMG. Other AMG methods, such as classical AMG and aggregation-based AMG, can be viewed as approximations to the energy-min AMG. The second approach is outlined in $\$ 6$. The main idea is to construct quasioptimal coarse space by piecing together the low-end eigenspaces of some appropriately defined local operators or matrices. This approach can be used to provide quasi-optimal coarse spaces for various AMG methods, including the standard geometric multigrid method, the classic AMG, energy-minimization AMG, unsmoothed and smoothed aggregation AMG, and spectral AMGe. As a simple example, this method relies on the fact that $n_{c}=J m$ dimensional low-end eigenspace of an operator can be well approximated by gluing together $J$-pieces of $m$-dimensional low-end eigenspaces for some carefully chosen local operators. Here $m$ is a very small integer. For example, $m=1$ for the for the Laplacian operator and $m=3$ (resp. $m=6$ ) for 2-dimensional (resp. 3-dimensional) linear elasticity operator. This important property of eigenspaces is closely related to the Weyl's Lemma on the asymptotic behavior of eigenvalues for elliptic boundary value problems, discussed in $\$ 2.3$

Most AMG methods are designed in terms of the adjacency graph of the coefficient matrix of a given linear algebraic system. In $\$ 7$, we give a brief description of graph theory and the adjacency graph of a sparse matrix. One highlight in $\$ 7$ 
is the concept of M-matrix relative. This simple tool is instrumental in the design and analysis of the classical AMG method.

One important step in the design of most AMG methods is to zero out some entries of the coefficient matrix $A$ by using the concept of strength of connections to get a filtered matrix $\tilde{A}$, which is equivalent to dropping out the weakly connected edges in the adjacency graph $\mathcal{G}(A)$ to get $\mathcal{G}(\tilde{A})$. Several definitions of strength functions are introduced in $\$ 8$ to describe the strength of connection. In $\$ 9$, the graph $\mathcal{G}(\tilde{A})$ is then coarsened by either keeping a maximal independent set (MIS) as a coarse vertex set $C$ and the dropping the rest of grid, or use aggregation/agglomeration. In $\$ 9$, some technical details are also given on the construction of coarse space by using degrees of freedom.

By using the aforementioned general approaches and theoretical techniques, we then motivate and present a number of AMG methods. Some of the highlights in the paper are outlined below.

We first give an overview of GMG and its relationship with AMG in $\$ 10$. After describing some details in a typical GMG method for linear finite element matrix, we argue that the geometric information used in defining a GMG is essentially the graph information of the underlying finite element grid (without using other geometric information such as coordinates of the grid points). This is a strong indication that at least some GMG method can be realized by a pure algebraic fashion: only using the stiffness matrix, an algebraic smoother, and the adjacency graph of the stiffness matrix. On the other hand, we prove that a GMG method can also be formally obtained by our general AMG approach presented in $\$ 6$. Furthermore, we use the example of AMGe in $\$ 10.4$ to demonstrate that geometric information on the grid can be effectively used to construct a geometry-based AMG.

In \$11, we give a detailed account on AMG methods based on energy-minimization. We first present our new theory that the optimal coarse space shown in the 2-level theory in $\$ 5$ can be actually obtained through trace-minimization (\$11.1). After proving that the trace-minimization can be equivalently formulated to an energyminimization problem in $\$ 11.1$, we then derive energy-min AMG method by seeking a set of locally supported coarse basis functions for energy-minimization.

Classical AMG, as the first class of AMG algorithms studied in the literature, will be presented in $\$ 12$. We derive and analyze this type of methods using the framework in $\$ 6$ and also the notion of M-matrix relatives introduced in $\$ 7.2$. We further discuss how a classical AMG method can be viewed as an approximation of energy-min AMG method.

Aggregation-based AMG will be presented in $\$ 13$. Again we derive and analyze this method using the framework in $\$ 6$. One remarkable feature of the aggregation AMG methods is their ease to preserve multi-dimensional near-null space, such as the rigid-body modes in linear elasticity.

To demonstrate how an AMG method addresses possible heterogeneous properties in a given problem, we devote $\$ 14$ to show how classical AMG is designed to address the difficulties arising from the discretized elliptic problems with strong 
discontinuous jumps or anisotropy in the coefficients of the underlying PDE. Finally we make some concluding remarks in $\$ 16$.

In \$15, we outline a class of AMG methods that attempt to choose the coarse spaces in a bootstrap and adaptive fashion. This line of AMG algorithms do not fall into the theoretical frameworks presented in this paper, but they provide a practical approach to generalize many existing AMG techniques to a more general class of problems.

We conclude these introductory remarks by a brief summary of the acronyms used in different $\mathrm{AMG}$ algorithms reviewed in this paper.

1 Aggregation-based AMG

- Unsmoothed aggregation

UA-AMG

- Smoothed aggregation

SA-AMG

2 Bootstrap \& Adaptive AMG

- Classical

$\alpha$ AMG

- Smoothed aggregation

$\alpha$ SA-AMG

- Bootstrap AMG

BAMG

3 Element-based AMG

AMGe

4 Spectral AMGe

$\rho \mathrm{AMGe}$

\section{Model problems and discretization}

While AMG has found applications to a wide range of linear algebraic systems, its development has been mainly motivated by the solution of systems arising from the discretization of partial differential equations by finite element, finite difference or other numerical methods. In this section, we will discuss a model of second order elliptic boundary problem, their finite difference and finite element discretization and relevant properties of the relevant underlying differential operators and their discretization.

\subsection{Model elliptic PDE operators}

We consider the following boundary value problems

$$
\mathcal{L} u=-\nabla \cdot \alpha(x) \nabla u=f, \quad x \in \Omega
$$

where $\alpha: \Omega \mapsto \mathbb{R}^{d \times d}$ is an SPD matrix function satisfying

$$
\alpha_{0}\|\xi\|^{2} \leq \xi^{T} \alpha(x) \xi \leq \alpha_{1}\|\xi\|^{2}, \quad \xi \in \mathbb{R}^{d} .
$$

for some positive constants $\alpha_{0}$ and $\alpha_{1}$. Here $d=1,2,3$ and $\Omega \subset \mathbb{R}^{d}$ is a bounded domain with boundary $\Gamma=\partial \Omega$.

A variational formulation for (2.1) is as follows: Find $u \in V$ such that

$$
a(u, v)=(f, v), \quad \forall v \in V .
$$


Here

$$
a(u, v)=\int_{\Omega}(\alpha(x) \nabla u) \cdot \nabla v, \quad(f, v)=\int_{\Omega} f v .
$$

and $V$ is a Sobolev space that can be chosen to address different boundary conditions accompanying the equation 2.1. One case is the mixed boundary conditions:

$$
\begin{aligned}
u & =0, & x \in \Gamma_{D}, \\
(\alpha \nabla u) \cdot n & =0, & x \in \Gamma_{N},
\end{aligned}
$$

where $\Gamma=\Gamma_{D} \cup \Gamma_{N}$. The pure Dirichlet problem is when $\Gamma_{D}=\Gamma$ while the pure Neumann problem is when $\Gamma_{N}=\Gamma$. We thus have $V$ as

$$
V=\left\{\begin{array}{l}
H^{1}(\Omega)=\left\{v \in L^{2}(\Omega): \partial_{i} v \in L^{2}(\Omega), i=1: d\right\} \\
H_{D}^{1}(\Omega)=\left\{v \in H^{1}(\Omega):\left.v\right|_{\Gamma_{D}}=0\right\} .
\end{array}\right.
$$

When we consider a pure Dirichlet problem, $\Gamma_{D}=\Gamma$, we denote the space by $V=H_{0}^{1}(\Omega)$. In addition, for pure Neumann boundary conditions, the following condition is added to assure the existence of the solution to (2.3):

$$
\int_{\Omega} f=0
$$

One most commonly used model problem is when

$$
\alpha(x)=1, \quad x \in \Omega,
$$

which corresponds to the Poisson equation

$$
-\Delta u=f .
$$

This simple problem provides a good representative model for isotropic problems.

There are other two cases that are of special interests. The first case is when $\alpha$ is a scalar and it has discontinuous jumps such as

$$
\alpha(x)= \begin{cases}\epsilon, & x \in \Omega_{1}, \\ 1, & x \in \Omega_{2} .\end{cases}
$$

The second case is when $\alpha$ is a diagonal matrix such as (for $d=2$ ):

$$
\alpha(x)=\left(\begin{array}{ll}
1 & 0 \\
0 & \epsilon
\end{array}\right)
$$

which corresponds to the following operator

$$
-u_{x x}-\epsilon u_{y y}=f .
$$

In both cases above, we assume that $\epsilon$ is sufficiently small to investigate the robustness of algorithms with respect to discontinuous jumps and an-isotropy. 


\subsection{Examples of finite difference and finite element discretizations}

As an illustrative example, we consider a finite difference discretization of the Poisson equation (2.8) with pure Dirichlet boundary conditions on the unit square $\Omega=(0,1) \times(0,1)$. We consider a uniform triangulation of $\Omega$ (see the two left figures in Fig 2.1) and we set

$$
\left(x_{i}, y_{j}\right)=\left(\frac{i}{n+1}, \frac{j}{n+1}\right), \quad u_{i, j} \approx u\left(x_{i}, y_{j}\right), \quad(i, j=0, \cdots, n+1) .
$$
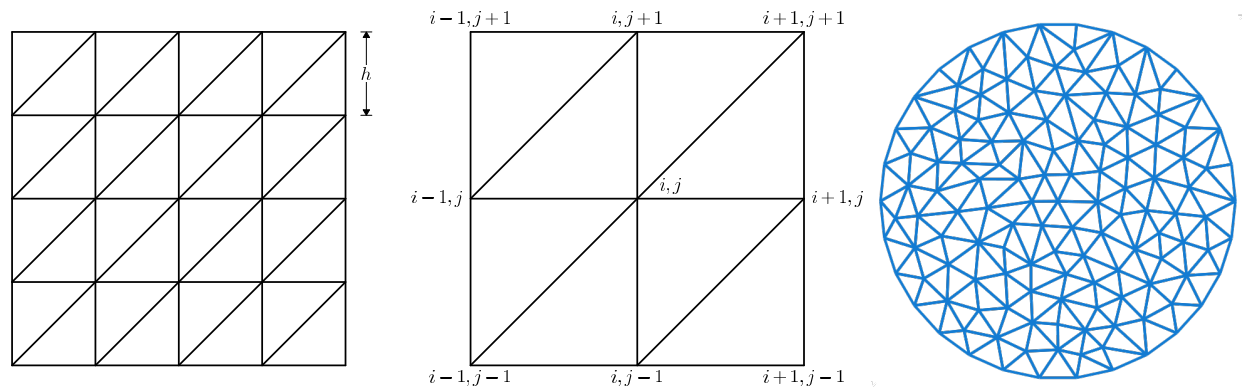

Figure 2.1. Regular (uniform) triangulations for the unit square (left and center) and unstructured mesh approximating the unit disk (right).

We use the standard center difference approximation to the Laplacian operator

$$
(-\Delta u)\left(x_{i}, y_{j}\right) \approx \frac{4 u_{i, j}-u_{i+1, j}-u_{i-1, j}-u_{i, j+1}-u_{i, j-1}}{h^{2}} .
$$

The finite difference scheme is then given by

$$
4 u_{i, j}-\left(u_{i+1, j}+u_{i-1, j}+u_{i, j+1}+u_{i, j-1}\right)=h^{2} f_{i, j},
$$

where

$$
f_{i, j}=f\left(x_{i}, y_{j}\right)
$$

and $u_{i, j} \approx u\left(x_{i}, y_{j}\right)$. The approximations $u_{i, j}$ are found by solving a linear system. We order the points $\left(x_{i}, y_{j}\right)$ lexicographically and we have for $k=1, \ldots, n^{2}$,

$$
k=(j-1) n+i, \quad x_{k}^{h}=\left(x_{i}, y_{j}\right), \quad \mu_{k}=u_{i, j}, \quad 1 \leq i, j \leq n,
$$

We can then write (2.12) as

$$
A \mu=b,
$$

where

$$
A=\operatorname{tridiag}(-I, B,-I) \text {, and } B=\operatorname{tridiag}(-1,4,-1) .
$$

A slightly different scheme is obtained using more of the neighboring points of $\left(x_{i}, y_{j}\right)$. We can build an approximation using 8 points $\left(x_{i \pm 1}, y_{j \pm 1}\right)$ together with the 
"center" point $\left(x_{i}, y_{j}\right)$. As a result we have the 9-point finite difference scheme as follows:

$$
\begin{aligned}
8 \mu_{i, j} & -\mu_{i-1, j}-\mu_{i+1, j}-\mu_{i, j-1}-\mu_{i, j+1} \\
& \quad-\mu_{i-1, j-1}-\mu_{i+1, j-1}-\mu_{i-1, j+1}-\mu_{i+1, j+1}=2 h^{2} f_{i, j} .
\end{aligned}
$$

Again, if we order $\left(x_{i}, y_{j}\right)$ lexicographically, then 2.17) is the linear system 2.15) corresponding to the 9-point finite difference discretization of the Laplace equation with

$$
A=\operatorname{tridiag}(-C, B,-C) \text { with } B=\operatorname{tridiag}(-1,8,-1), C=\operatorname{tridiag}(1,1,1) .
$$

We now give an example of finite element discretization. Given a triangulation $\mathcal{T}_{h}$ for $\Omega$, such as that given in Figure 2.1, let $V_{h} \subset V$ be a finite element space consisting of piecewise linear (or higher order) polynomials with respect to the triangulation $\mathcal{T}_{h}$. The finite element approximation of the variational problem 2.3 is: Find $u_{h} \in V_{h}$ such that

$$
a\left(u_{h}, v_{h}\right)=\left(f, v_{h}\right), \quad \forall v_{h} \in V_{h} .
$$

Assume $\left\{\phi_{i}\right\}_{i=1}^{N}$ is the nodal basis of $V_{h}$, namely, $\phi_{i}\left(x_{j}\right)=\delta_{i j}$ for any nodes $x_{j}$. We write $u_{h}(x)=\sum_{j=1}^{N} \mu_{j} \phi_{j}(x)$ the equation 2.19) is then equivalent to

$$
\sum_{j=1}^{N} \mu_{j} a\left(\phi_{j}, \phi_{i}\right)=\left(f, \phi_{i}\right), \quad j=1,2, \cdots, N,
$$

which is a linear system of equations:

$$
A \mu=b, \quad(A)_{i j}=a\left(\phi_{j}, \phi_{i}\right), \quad \text { and } \quad(b)_{i}=\left(f, \phi_{i}\right) .
$$

Here, the matrix $A$ is known as the stiffness matrix of the nodal basis $\left\{\phi_{i}\right\}_{i=1}^{N}$.

For $d=2$ and the special uniform triangulation as shown on the in Figure 2.1. this stiffness matrix for the Laplacian operator turns out to be exactly the one given by (2.12). This special case is an example of the close relationship between finite difference and finite element methods.

We note that the finite element method is based on the variational formulation (2.3), whereas the finite difference method is not. In the development of AMG method, however, variational method is also used to derive coarse level equations for finite difference methods.

For any $T \in \mathcal{T}_{h}$, we define

$$
\bar{h}_{T}=\operatorname{diam}(T), \quad h_{T}=|T|^{\frac{1}{d}}, \quad \underline{h}_{T}=2 \sup \{r>0: B(x, r) \subset T \text { for } x \in T\} .
$$

We say that the mesh $\mathcal{T}_{h}$ is shape regular if there exists a uniformly bounded constant $\sigma \geq 1$ such that

$$
\underline{h}_{T} \leq h_{T} \leq \bar{h}_{T} \leq \sigma \underline{h}_{T}, \quad \forall T \in \mathcal{T}_{h} .
$$

And we call $\sigma$ the shape regularity constant. 


\subsection{Spectral properties}

Let $h=\max _{T \in \mathcal{T}_{h}} \bar{h}_{T}$, with $\bar{h}_{T}$ defined in (2.21). We say that the mesh $\mathcal{T}_{h}$ is quasi-uniform if there exists a uniformly bounded constant $C>0$ such that

$$
\frac{h}{\underline{h}_{T}} \leq C .
$$

\subsection{Spectral properties}

We now discuss the spectral properties of the partial differential operator $\mathcal{L}$ given in (2.1).

We recall the well-known Courant-Fischer min-max principle (Courant and Hilbert 1924) for eigenvalues of symmetric matrices.

Theorem 2.1. Let $T$ be a $n \times n$ symmetric matrix with respect to $(\cdot, \cdot)_{*}$, and $\left\{\lambda_{j}, \zeta_{j}\right\}$ are its eigenpairs with $\lambda_{1} \leq \lambda_{2} \leq \cdots \leq \lambda_{n}$, then

$$
\lambda_{k}=\min _{\operatorname{dim} W=k} \max _{x \in W, x \neq 0} \frac{(T x, x)_{*}}{(x, x)_{*}},
$$

where the minimum is achieved if

$$
W=\operatorname{span}\left\{\zeta_{j}: j=1: k\right\},
$$

and

$$
\lambda_{k}=\max _{\operatorname{dim} W=n-k+1} \min _{x \in W, x \neq 0} \frac{(T x, x)_{*}}{(x, x)_{*}},
$$

where the maximum is achieved if

$$
W=\operatorname{span}\left\{\zeta_{j}: j=k: n\right\} .
$$

Next, we recall Theorem 1 in (Fan 1949) which is known as Ky-Fan trace minimization principle.

Theorem 2.2. We suppose $T$ is symmetric with respect to $(\cdot, \cdot)_{*}$, and $\left\{\lambda_{j}, \zeta_{j}\right\}$ are its eigenpairs with $\lambda_{1} \leq \lambda_{2} \leq \cdots \leq \lambda_{n}$, then

$$
\min _{P \in \mathbb{R}^{n \times k}, P^{*} P=I} \operatorname{trace}\left(P^{*} T P\right)=\sum_{j=1}^{k} \lambda_{j} .
$$

Furthermore, the minimum is achieved when

$$
\operatorname{range}(P)=\operatorname{span}\left\{\zeta_{j}\right\}_{j=1}^{k} \text { and } P^{*} P=I .
$$

Here $P^{*} \in \mathbb{R}^{k \times n}$ is the adjoint of $P$ corresponds to $(\cdot, \cdot)_{*}$ inner product, namely

$$
\left(P^{*} u, v\right)=(u, P v)_{*}, \quad \text { for all } u \in \mathbb{R}^{n}, v \in \mathbb{R}^{k} .
$$

Finally, following (Xu 1992), we use the notation $a \lesssim b$ to represent the existence of a generic positive constant $C$, which is independent of important parameters, such as problem size, anisotropic ratio, or other, and such that $a \leq C b$. Furthermore, we write $a \equiv b$ iff $a \lesssim b$ and $b \lesssim a$. 
Theorem 2.3. The PDE operator $\mathcal{L}$ has a complete set of eigenfunctions $\left(\varphi_{k}\right)$ and nonnegative eigenvalues

$$
0 \leq \lambda_{1} \leq \lambda_{2} \leq \ldots
$$

such that

$$
\mathcal{L} \varphi_{k}=\lambda_{k} \varphi_{k}, \quad k=1,2,3 \ldots
$$

$1 \lim _{k \rightarrow \infty} \lambda_{k}=\infty$.

$2\left(\varphi_{i}\right)$ forms an orthonomal basis of $V$ as well as for $L^{2}(\Omega)$.

Furthermore

1 For pure Neumann problem, $\lambda_{1}=0$ and $\varphi_{1}$ is the constant function.

2 For pure Dirichlet problem, $\lambda_{1}>0$ is simple and $\varphi_{1}$ does not change sign.

We have the well-known Weyl's estimate on the asymptotic behavior of the Laplacian operator (Weyl 1911, Weyl 1912, Reed and Simon 1978).

Lemma 2.4. (Weyl's law) Assume that $\Omega$ is contented (which means $\Omega$ can be approximated by unions of cubes in $\mathbb{R}^{d}$, see (Reed and Simon 1978, page 271) for the exact definition). For homogeneous Dirichlet boundary condition, the eigenvalues of the pure Laplacian operator satisfy:

$$
\lim _{k \rightarrow \infty} \frac{\lambda_{k}}{k^{\frac{2}{d}}}=w_{\Omega}, \text { with } \quad w_{\Omega}=\frac{(2 \pi)^{2}}{\left[\omega_{d} \operatorname{Vol}(\Omega)\right]^{\frac{2}{d}},}
$$

where $\omega_{d}$ is a volume of the unit ball in $\mathbb{R}^{d}$, and the eigenvalues of the operator $\mathcal{L}$ given in (2.1) satisfy:

$$
\lambda_{k} \equiv k^{\frac{2}{d}}, \quad \forall k \geq 1 .
$$

Next, we extend the above Weyl's law to discretized PDE operators. The following theorem gives a discrete version of the Weyl's law for the finite element discretization. Further details on such a result are found in $(\mathrm{Xu}$, Zhang and Zikatanov 2016b).

Theorem 2.5. Let $V_{h} \subset H_{0}^{1}(\Omega)$ be a family of finite element spaces on a quasiuniform mesh with $\operatorname{dim} V_{h}=N$. Consider the discretized operator of 2.1]

$$
\mathcal{L}_{h}: V_{h} \mapsto V_{h}, \quad\left(\mathcal{L}_{h} u, v\right)=a(u, v), \quad \forall u, v \in V_{h},
$$

and its eigenvalues:

$$
\lambda_{h, 1} \leq \lambda_{h, 2} \leq \cdots \lambda_{h, N}
$$

Then, for all $1 \leq k \leq N$, there exists a constant $C_{w}>0$ independent of $k$ such that we have the following estimates:

$$
\lambda_{k} \leq \lambda_{h, k} \leq C_{w} \lambda_{k} .
$$

and

$$
\lambda_{h, k} \equiv k^{2 / d} \text {. }
$$




\subsection{Properties of finite element matrices}

\subsection{Properties of finite element matrices}

The main algebraic property for the stiffness matrices given by $(2.20)$ is that: it is sparse with $O(N)$ nonzeros, symmetric-postive definite (for both Dirichlet and mixed boundary conditions) and semi-definite for pure Neumann boundary conditions; Its eigenvalues satisfy the discrete Weyl's law.

For simplicity, we will only consider the pure Dirichlet boundary conditions in the rest of this section.

Lemma 2.6. The stiffness matrix $A$ given by 2.20 has the following properties:

1 The condition number of $A$, defined by the ratio of the extreme eigenvalues of $A$,

$$
\kappa(A)=\frac{\lambda_{\max }(A)}{\lambda_{\min }(A)},
$$

satisfies

$$
\kappa(A) \equiv h^{-2} .
$$

Furthermore,

$$
\lambda_{\min }(A) \equiv h^{2} \text { and } \lambda_{\max }(A) \equiv 1 .
$$

2 The discrete version of the Weyl's law holds:

$$
\lambda_{k}(A) \equiv\left(\frac{k}{N}\right)^{2 / d} \text {. }
$$

We next discuss some more refined spectral properties of finite element stiffness matrices from uniform grids for the unit square domain $\Omega=(0,1) \times(0,1)$ for $d=2$. We begin with the Poisson equation. It is easy to derive a closed-form solution of the eigenpairs of $A$ given by (2.15) and we have:

$$
\lambda_{k l}(A)=4\left(\sin ^{2} \frac{k \pi}{2(n+1)}+\sin ^{2} \frac{l \pi}{2(n+1)}\right),
$$

and

$$
\phi_{i j}^{k l}=\sin \frac{k i \pi}{n+1} \sin \frac{l j \pi}{n+1}, \quad 1 \leq i \leq n, \quad 1 \leq j \leq n .
$$

Consider now the important case of anisotropic problem (2.11). We order the vertices of the triangulation lexicographically, and, as before, denote them by $\{(i h, j h)\}_{i, j=0}^{n}$. The stiffness matrix then is

$$
A=\operatorname{tridiag}(-I, B,-I) \quad \text { with } \quad B=\operatorname{tridiag}(-\epsilon, 2(1+\epsilon),-\epsilon) .
$$

Obviously,

$$
A=I \otimes B+C \otimes I \quad \text { with } \quad C=\operatorname{tridiag}(-1,0,-1)
$$


and it is easily verified that

$$
\lambda_{i}(B)=2(1+\epsilon)-2 \epsilon \cos \frac{i \pi}{(n+1)}, \quad \lambda_{j}(C)=-2 \cos \frac{j \pi}{(n+1)}, \quad 1 \leq i, j \leq N .
$$

which leads to the following expression for the eigenvalues

$$
\lambda_{i j}(A)=4 \epsilon \sin ^{2} \frac{i \pi}{2(n+1)}+4 \sin ^{2} \frac{j \pi}{2(n+1)} .
$$

and the corresponding eigen-vectors

$$
\phi_{i j}^{k \ell}=\sin \frac{k i \pi}{n+1} \sin \frac{\ell j \pi}{n+1} .
$$

\section{Linear vector spaces and duals}

In this paper, we will mainly consider linear system of equation of the following form:

$$
A u=f
$$

Here

$$
\begin{gathered}
A: V \mapsto V^{\prime}, \\
f \in V^{\prime},
\end{gathered}
$$

$V$ is a finite-dimensional linear vector space and $V^{\prime}$ is the dual of $V$. If we use the notation $\langle\cdot, \cdot\rangle$ to denote the pairing between $V^{\prime}$ and $V$, we can write (3.1) in a variational form: Find $u \in V$ such that

$$
a(u, v)=\langle f, v\rangle, \quad \forall v \in V
$$

where

$$
a(u, v)=\langle A u, v\rangle
$$

\subsection{Dual and inner product}

For convenience of exposition, we will assume that $V$ is equipped with an inner product $(\cdot, \cdot)$. By Riesz representation theorem, for any $f \in V^{\prime}$, there is a unique $u \in V$ such that

$$
(u, v)=\langle f, v\rangle, \quad \forall v \in V .
$$

It is through this representation, we will take $V^{\prime}=V$. In the rest of this paper, for convenience, we will always assume that $V^{\prime}=V$ for any finite dimensional vector space $V$. As a result, we have

$$
V^{\prime \prime}=\left(V^{\prime}\right)^{\prime}=V^{\prime}=V
$$

Thanks to the identification $V^{\prime}=V$ via (3.5), the identities in 3.6 are clear without any ambiguity. 


\subsection{Dual and inner product}

We would like to point out that, in an abstract discussion of all iterative methods for problem (3.1), it suffice to use the abstract dual pairing $\langle\cdot, \cdot\rangle$ without having to introducing an inner product $(\cdot, \cdot)$ on $V$. But we find that the use an inner product is convenient for exposition as we shall see later. We further point out that we will not use an inner product to identify $V^{\prime}=V$ for any infinite dimensional vector space in this paper.

If $\left\{\phi_{i}\right\}_{i=1}^{N}$ is a basis of $V$, we will always choose a basis, $\left\{\psi_{i}\right\}_{i=1}^{N}$, of $V^{\prime}$ that is dual to the basis of $V$. Namely

$$
\left(\psi_{j}, \phi_{i}\right)=\delta_{i j}, \quad 1 \leq i, j \leq N .
$$

Such a dual basis will only used for theoretical consideration and it will not be used in actual implementation of any algorithms.

We will only consider two kinds of linear vector spaces: The first kind is $V=\mathbb{R}^{n}$ and the inner product is just the dot product

$$
(u, v)_{\ell^{2}}=\sum_{i=1}^{n} u_{i} v_{i}, \quad \forall u=\left(u_{i}\right), v=\left(v_{i}\right) \in \mathbb{R}^{n},
$$

A canonical basis of $\mathbb{R}^{N}$ is formed by the column vectors of the identity matrix, $\left\{e_{i}\right\}_{i=1}^{N}$, it is easy to see that its dual basis of $\left(\mathbb{R}^{N}\right)^{\prime}=\mathbb{R}^{N}$ is just the original basis $\left\{e_{i}\right\}_{i=1}^{N}$ itself.

The second kind is a finite dimensional functional subspace of $L^{2}(\Omega)$ for a given domain $\Omega \subset \mathbb{R}^{d}(1 \leq d \leq 3)$, equipped with the $L^{2}$ inner product:

$$
(u, v)=\int_{\Omega} u(x) v(x) .
$$

One commonly used linear vector space is a finite element space $V_{h}$ and oftentimes the nodal basis functions $\left\{\phi_{i}\right\}_{i=1}^{N}$ are used as a basis. In this case, the dual basis, $\left\{\psi_{i}\right\}_{i=1}^{N}$, of $V^{\prime}$ are not the original nodal basis functions anymore, but rather, a set of functions (which are usually globally supported) that satisfy (3.7). This set of dual basis functions is usually needed in deriving matrix representation of operators between various spaces and their duals, but they are not needed in the actual implementation of relevant algorithms.

For a linear operator

$$
L: V \mapsto V,
$$

its adjoint:

$$
L^{\prime}: V \mapsto V,
$$

is defined as follows

$$
\left(L^{\prime} u, v\right)=(u, L v), \quad u, v \in V .
$$

Since $V$ plays the role of both $V$ and its dual $V^{\prime}$, the notion (3.8) and (3.9) can have four different meanings: 
1. If $L: V \mapsto V$, then $L^{\prime}: V^{\prime} \mapsto V^{\prime}$;

2. If $L: V \mapsto V^{\prime}$, then $L^{\prime}: V \mapsto V^{\prime}$;

3. If $L: V^{\prime} \mapsto V$, then $L^{\prime}: V^{\prime} \mapsto V$;

4. If $L: V^{\prime} \mapsto V^{\prime}$, then $L^{\prime}: V \mapsto V$.

Thanks to the identification we made between $V^{\prime}$ and $V$ through (3.5), the definition (3.10) is applicable to all the above four different cases.

If $V=\mathbb{R}^{n}$ and $(u, v)=(u, v)_{\ell^{2}}, L^{\prime}=L^{T}$, namely the matrix transpose. We say that an operator $A: V \mapsto V^{\prime}$ is symmetric positive definite (SPD) if

$$
A^{\prime}=A, \quad(A v, v)>0 \quad \forall v \in V \backslash\{0\} .
$$

When $A$ is SPD, it defines another inner product $(\cdot, \cdot)_{A}$ on $V$ :

$$
(u, v)_{A}=(A u, v), \quad u, v \in V
$$

and a corresponding norm

$$
\|v\|_{A}=(v, v)_{A}^{1 / 2}, \quad v \in V
$$

We use the superscript “*” for the adjoint operator with respect to $(\cdot, \cdot)_{A}$, i.e.

$$
(B u, v)_{A}=\left(u, B^{*} v\right)_{A} .
$$

It is easy to see that

$$
(B A)^{*}=B^{\prime} A,
$$

and $(B A)^{*}=B A$ if and only if $B^{\prime}=B$.

\subsection{Matrix representation}

Let $V_{c} \subset V$ be a subspace and consider the inclusion operator $\iota_{c}: V_{c} \mapsto V$. Assume that $\left\{\phi_{i}^{c}\right\}_{i=1}^{n_{c}}$ and $\left\{\phi_{i}\right\}_{i=1}^{n}$ are basis functions of $V_{c}$ and $V$ respectively, the matrix representation of $l_{c}$ is a matrix

$$
P: \mathbb{R}^{n_{c}} \mapsto \mathbb{R}^{n} \text { satisfying }\left(\phi_{1}^{c}, \ldots, \phi_{n_{c}}^{c}\right)=\left(\phi_{1}, \ldots, \phi_{n}\right) P .
$$

The identity written above is a shorthand for the expansion of the basis in $V_{c}$ via the basis in $V$ :

$$
\phi_{k}^{c}=\sum_{j=1}^{n} p_{j k} \phi_{j}, \quad P=\left(p_{j k}\right), \quad k=1, \ldots, n_{c}, \quad j=1, \ldots, n .
$$

What is the matrix representation of $\imath_{c}^{\prime}: V^{\prime} \mapsto V_{c}^{\prime}$ ? Although we have $V^{\prime}=V$ and $V_{c}^{\prime}=V_{c}$, we need to use dual bases $\left\{\psi_{i}^{c}\right\}_{i=1}^{n_{c}} \subset V_{c}^{\prime}$ and $\left\{\psi_{i}\right\}_{i=1}^{n} \subset V^{\prime}$ respectively. With respect to these dual bases, the matrix representation of $t_{c}^{\prime}$ is simply $P^{T}$ (the transpose of $P$ ) since it is easy to verify that

$$
\left(\imath_{c}^{\prime} \psi_{1}, \ldots, \iota_{c}^{\prime} \psi_{n}\right)=\left(\psi_{1}^{c}, \ldots, \psi_{n_{c}}^{c}\right) P^{T}
$$




\subsection{Matrix representation}

Consider now a linear operator

$$
A: V \mapsto V
$$

There are two different ways to get a matrix representation of $A$ because $V$ plays two roles here. First $V$ is $V$ itself, and secondly $V=V^{\prime}$. For the first case, we use the same basis $\left\{\phi_{i}\right\}$ for $V$ as the domain of $A$ and $V$ as the range of $A$. In this case, the matrix representation of $A$ is the matrix

$$
\hat{A} \in \mathbb{R}^{n \times n} \text { satisfying }\left(A \phi_{1}, \ldots, A \phi_{n}\right)=\left(\phi_{1}, \ldots, \phi_{n}\right) \hat{A} .
$$

In the second case, we use the basis $\left\{\phi_{i}\right\}$ for $V$ as the domain space of $A$, but use the dual basis $\left\{\psi_{i}\right\}$ for $V^{\prime}=V$ as the range space of $A$. In this case, the matrix representation of $A$ is the matrix

$$
\tilde{A} \in \mathbb{R}^{n \times n} \text { satisfying }\left(A \phi_{1}, \ldots, A \phi_{n}\right)=\left(\psi_{1}, \ldots, \psi_{n}\right) \tilde{A} .
$$

It is easy to see that

$$
\tilde{A}=\left(\left(A \phi_{j}, \phi_{i}\right)\right)
$$

and

$$
\tilde{A}=M \hat{A}, \quad M=\left(\left(\phi_{j}, \phi_{i}\right)\right) .
$$

The matrix $\tilde{A}$ in $(3.17)$ is often called the stiffness matrix of $A$ and the matrix $M$ in $(3.18)$ is called the mass matrix.

In the early multigrid literature, a discrete inner product equivalent to the $L^{2}$ inner product was often introduced for finite element spaces so that the corresponding mass matrix becomes diagonal. But if we view the underlying finite element operator as in (3.14) in a slightly different way:

$$
A: V \mapsto V^{\prime},
$$

and we will then see easily that the introduction of the discrete $L^{2}$ inner product is not necessary.

If $V=\mathbb{R}^{n}$ and we choose the canonical basis $\left\{e_{i}\right\}$ for $V$, we would not encounter the mass matrix problem as in the functional space case since in this case $\left\{e_{i}\right\}$ is also the dual basis of $V^{\prime}$. This is certainly convenient, but such a convenience tends to hide some subtle but important difference between various vectors and matrices in a given problem and the objects (functions) that they represent.

Given a matrix $A \in \mathbb{R}^{n \times n}$, we can either view it as

$$
A: \mathbb{R}^{n} \mapsto \mathbb{R}^{n},
$$

or

$$
A: \mathbb{R}^{n} \mapsto\left(\mathbb{R}^{n}\right)^{\prime} .
$$

As it turns out, when $A$ is obtained from the discretization of partial differential 
equations, 3.21) is more informative than 3.20). Hence we write a matrix equation

$$
A x=b
$$

it is sometimes helpful to view that $x$ and $b$ live in two "different" spaces:

$$
x \in \mathbb{R}^{n} \text { and } b \in\left(\mathbb{R}^{n}\right)^{\prime} .
$$

\subsection{Eigenvalues and eigenvectors}

Let us discuss briefly on eigenvalues and eigenvectors for symmetric operator $T$ : $V \mapsto V$. If $(\lambda, \phi)$ is an eigen-pair of $T$,

$$
T \phi=\lambda \phi .
$$

Then it is easy to see that $(\lambda, \tilde{\phi})$ is an eigenpair of the matrix representation $\tilde{T}$ of $T$ :

$$
\tilde{T} \tilde{\phi}=\lambda \tilde{\phi} .
$$

Here $\tilde{\phi}=\in \mathbb{R}^{n}$ is the vector representation of $\phi$ :

$$
\phi=\left(\phi_{1}, \ldots, \phi_{n}\right) \tilde{\phi} .
$$

We note that for an operator $A$ defined in (3.14), we need to be cautious when we talk about eigenvalues of $A$. Although we identity $V^{\prime}=V$ through (3.5), $V^{\prime}$ and $V$ play two different roles and thus $A$ is essentially a mapping between two "different" spaces $V$ and $V^{\prime}$ and the spectrum of $A$ should be defined carefully. But if we consider a symmetric operator

$$
R: V^{\prime} \mapsto V
$$

Then $R A: V \mapsto V$ is an operator that is symmetric with respect to $A$-inner product. In this case if $(\lambda, \phi)$ is an eigenpair of $R A$, then $(\lambda, \tilde{\phi})$ is an eigenpair of $\tilde{R} \tilde{A}$ (that is equal to the matrix representation of $R A$ )

If we consider a trivial identification operator

$$
\jmath: V^{\prime} \mapsto V \text { such that } \jmath \psi_{i}=\psi_{i}, \quad \forall i .
$$

Namely $j v=v$ for all $v \in V^{\prime}=V$. It is easy to see that the matrix representation of $J$ is the inverse of the mass matrix $M=\left(\left(\phi_{j}, \phi_{i}\right)\right)$, namely

$$
\tilde{\jmath}=M^{-1} \text {. }
$$

Using this identification operator, the operator $j A: V \mapsto V$ is a symmetric operator from $V$ to $V$. We can then talk about its spectrum. For example, if $(\lambda, \phi)$ is an eigen-pair of $\jmath A$, then $(\lambda, \tilde{\phi})$ satisfies

$$
\tilde{A} \tilde{\phi}=\lambda M \tilde{\phi} .
$$

This is often the generalized eigenvalue problem appearing in finite element analysis. 


\subsection{Bibliographical notes}

Although for all $v \in V, J A v=A v$ because of the identification introduced above, $J A$ and $A$ are, strictly speaking, two different operators: they have two different ranges and their matrix representations are different.

The discussions above, although simple, may sound a little bit confusing at first glance, but an unambiguous understanding and clarification of these concepts and the underlying subtleties will be helpful for the presentation of algebraic multigrid methods in the rest of this article. For more detailed discussions on relevant topics, we refer to (Xu 2016).

\subsection{Bibliographical notes}

For a general reading on the basic linear algebra materials used here, we refer to (Halmos 1974, Xu 1992, Xu 2016). In particular, for a more detailed discussion related to dual spaces and matrix representations, we refer to (Xu 1992, Xu 2016).

\section{Basic iterative methods}

We now consider linear iterative methods for solving 2.20). We will focus on two most commonly used algorithms, namely Jacobi and Gauss-Seidel methods. Let us first give a brief introduction of linear iterative methods in a more general setting. Recall the basic problem under consideration: Given a finite dimensional vector space $V$ equipped with an inner product $(\cdot, \cdot)$, we consider

$$
A u=f,
$$

where $A: V \mapsto V^{\prime}$ is symmetric positive definite (SPD) and $V^{\prime}$ is the dual of $V$. As mentioned in $\$ 3$, we will identify $V^{\prime}=V$ through an inner product $(\cdot, \cdot)$.

\subsection{Basic iterative methods}

A general linear iterative method for solving (4.1) can be written as follows: given $u^{0} \in V$,

$$
u^{m}=u^{m-1}+B\left(f-A u^{m-1}\right), \quad m=1,2, \cdots,
$$

where $B: V^{\prime} \mapsto V$ is a linear operator which can be thought of as an approximate inverse of $A$.

Sometimes it is more desirable that the iterator $B$ is symmetric. If $B$ is not symmetric, there is a natural way to symmetrize it. Consider the following iteration

$$
\begin{cases}u^{m-1 / 2} & =u^{m-1}+B\left(f-A u^{m-1}\right), \\ u^{m} & =u^{m-1 / 2}+B^{\prime}\left(f-A u^{m-1 / 2}\right) .\end{cases}
$$

The symmetrized iteration (4.3) can be written as

$$
u^{m}=u^{m-1}+\bar{B}\left(f-A u^{m-1}\right), \quad m=1,2, \cdots
$$

where

$$
\bar{B}=B^{\prime}+B-B^{\prime} A B,
$$


which satisfies

$$
I-\bar{B} A=(I-B A)^{*}(I-B A) .
$$

Obviously, $\rho(I-\bar{B} A)<0 \Longleftrightarrow \bar{B}>0 \Longleftrightarrow G \equiv\left(B^{\prime}\right)^{-1}+B^{-1}-A>0$.

Theorem 4.1. The following results hold

1 (4.3) converges $\Longleftrightarrow G>0 \Longrightarrow(4.2$ ) converges. Furthermore

$$
\|I-B A\|_{A}^{2}=\lambda_{\max }(I-\bar{B} A)=1-\left(\sup _{\|v\|_{A}=1}\left(\bar{B}^{-1} v, v\right)\right)^{-1}
$$

2 If $B^{\prime}=B, G>0 \Longleftrightarrow 4.2$ converges and, with $\eta=\lambda_{\min }(G)$,

$$
\frac{2 \eta}{\eta+1}(B v, v) \leq(\bar{B} v, v) \leq 2(B v, v), \quad v \in V .
$$

Exercise 4.2. Generalize Theorem 4.1 to the case that $A$ is SSPD.

\subsection{Jacobi and Gauss-Seidel methods}

For $A=\left(a_{i j}\right) \in \mathbb{R}^{n \times n}$, we write

$$
A=D+L+U
$$

where $D$ is the diagonal of $A, L$ and $U$ are the strict lower and upper triangular parts of $A$ respectively.

Given $\omega>0$, the (modified) Jacobi method can be written as (4.2) with

$$
B=\omega D^{-1}=\left(\omega^{-1} D\right)^{-1},
$$

and the resulting algorithm is as follows:

$\overline{\text { Algorithm } 1 \text { Modified Jacobi }}$
\[ \text { For } i=1: n, \quad x_{i}^{m}=x_{i}^{m-1}+\omega a_{i i}^{-1}\left(b_{i}-\sum_{j=1}^{n} a_{i j} x_{j}^{m-1}\right) . \]

The (modified) Gauss-Seidel method can be written as (4.2) with

$$
B=\left(\omega^{-1} D+L\right)^{-1}
$$

and the resulting algorithm is as follows:

The following result, which follows easily from Theorem 4.1, is well-known.

Theorem 4.3. The modified Jacobi method converges if and only

$$
0<\omega<\frac{2}{\rho\left(D^{-1} A\right)},
$$


Algorithm 2 Modified Gauss-Seidel Method

For $i=1: n, \quad x_{i}^{m}=x_{i}^{m-1}+\omega a_{i i}^{-1}\left(b_{i}-\sum_{j=1}^{i-1} a_{i j} x_{j}^{m}-\sum_{j=i}^{n} a_{i j} x_{j}^{m-1}\right)$.

and the modified Gauss-Seidel method converges if and only if

$$
0<\omega<2 \text {. }
$$

In practice, it is often easy to properly choose $\omega$ to satisfy (4.9) so that the modified Jacobi method is guaranteed to converge. In the rest of this paper, we may always assume that such a choice of $\omega$ is made. For Gauss-Seidel method, we will always choose $\omega=1$ (optimal SOR is not usually used in multigrid method). The Jacobi and Gauss-Seidel methods together with their convergence theory can be extended to block-matrices in a straightforward fashion.

\subsection{The method of subspace corrections}

We consider a sequence of spaces $V_{1}, \ldots, V_{J}$. These spaces, which will be known as auxiliary spaces, are not necessarily subspaces of $V$, but each of them is related to the original space $V$ by a linear operator

$$
\Pi_{k}: V_{k} \mapsto V .
$$

Our very basic assumption is that the following decomposition holds:

$$
V=\sum_{i=1}^{J} \Pi_{i} V_{i}
$$

This means that for any $v \in V$, there exists $v_{i} \in V_{i}$ (which may not be unique) such that

$$
v=\sum_{i=1}^{J} \Pi_{i} v_{i}
$$

Furthermore, we assume that each $V_{i}$ is equipped with an energy inner product $a_{i}(\cdot, \cdot)$. We define

$$
A_{i}: V_{i} \mapsto V_{i}^{\prime},
$$

by

$$
\left(A_{i} u_{i}, v_{i}\right)=a_{i}\left(u_{i}, v_{i}\right), \quad u_{i}, v_{i} \in V_{i}
$$

Let $\Pi_{i}^{\prime}: V^{\prime} \mapsto V_{i}^{\prime}$ be the adjoint of $\Pi_{i}$ :

$$
\left(\Pi_{i}^{\prime} f, v_{i}\right)=\left(f, \Pi_{i} v_{i}\right), \quad f \in V^{\prime}, v_{i} \in V_{i} .
$$

Let $P_{i}=\Pi_{i}^{*}: V \mapsto V_{i}$ be the adjoint of $\Pi_{i}$ with respect to the A-inner products:

$$
\left(P_{i} u, v_{i}\right)_{A_{i}}=\left(u, \Pi_{i} v_{i}\right)_{A}, u \in V, v_{i} \in V_{i} .
$$


The following identity holds

$$
\Pi_{i}^{\prime} A=A_{i} P_{i} .
$$

If $u$ is the solution of (4.1), by (4.14), we have

$$
A_{i} u_{i}=f_{i},
$$

where

$$
u_{i}=P_{i} u, \quad f_{i}=\Pi_{i}^{\prime} f
$$

This equation may be regarded as the restriction of (4.1) to $V_{i}$. We assume that each such $A_{i}$ has an approximate inverse or preconditioner:

$$
R_{i}: V_{i}^{\prime} \mapsto V_{i}
$$

The parallel subspace correction (PSC in short) method is (4.2) with $B=B_{p s c}$ given by

$$
B_{p s c}=\sum_{i=1}^{J} \Pi_{i} R_{i} \Pi_{i}^{\prime} .
$$

The successive subspace correction (SSC in short) method is defined as:

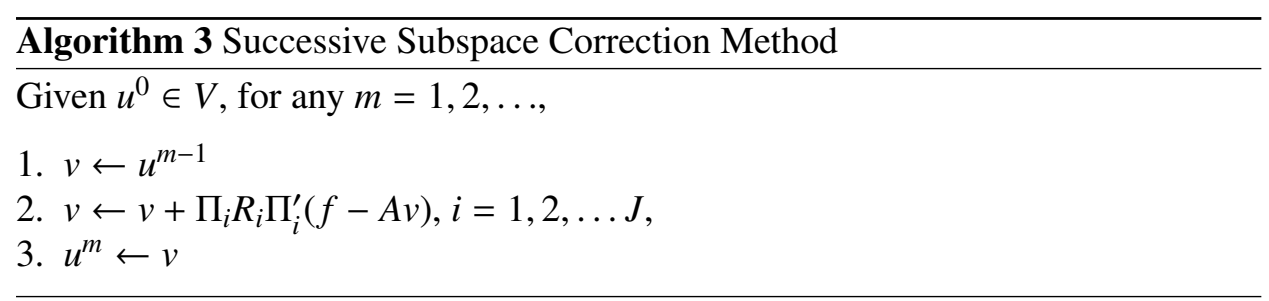

The Algorithm 3 is equivalent to (4.2) with $B=B_{s s c}$ given by

$$
I-B_{s s c} A=\left(I-T_{J}\right)\left(I-T_{J-1}\right) \ldots\left(I-T_{1}\right),
$$

where

$$
T_{i}=\Pi_{i} R_{i} \Pi_{i}^{\prime} A=\Pi_{i} R_{i} A_{i} P_{i} .
$$

Theorem 4.4. Assume that all $R_{k}$ are SPD. Then

$$
\left(B_{p s c}^{-1} v, v\right)=\min _{\sum_{i} \Pi_{i} v_{i}=v} \sum_{k=1}^{J}\left(R_{k}^{-1} v_{k}, v_{k}\right)
$$

with the unique minimizer given by

$$
v_{k}^{*}=R_{k} \Pi_{k}^{\prime} B_{p s c}^{-1} v .
$$




\subsection{The method of subspace corrections}

Theorem 4.5. Under the assumptions given above the following identity holds:

$$
\begin{aligned}
\left\|I-B_{s s c} A\right\|_{A}^{2} & =\left\|\left(I-T_{J}\right)\left(I-T_{J-1}\right) \ldots\left(I-T_{1}\right)\right\|_{A}^{2} \\
& =1-\frac{1}{1+c_{0}} \\
& =1-\frac{1}{c_{1}} .
\end{aligned}
$$

Here

$$
c_{0}=\sup _{\|v\|_{A}=1} c_{0}(v), \quad c_{1}=\sup _{\|\nu\|_{A}=1} c_{1}(v)=1+c_{0}
$$

and, with $w_{i}=\left(I-T_{i}^{-1}\right) \Pi_{i} v_{i}+\sum_{j=i+1}^{J} \Pi_{j} v_{j}$

$$
c_{0}(v)=\inf _{\sum_{i} \Pi_{i} v_{i}=v} \sum_{i=1}^{J}\left(T_{i} \bar{T}_{i}^{-1} T_{i}^{*} w_{i}, w_{i}\right)_{A},
$$

and

$$
c_{1}(v)=\left(\bar{B}_{s s c}^{-1} v, v\right)=\inf _{\sum_{i} \Pi_{i} v_{i}=v}\left(\bar{T}_{i}^{-1}\left(\bar{T}_{i} T_{i}^{-1} \Pi_{i} v_{i}+T_{i}^{*} w_{i}\right),\left(\bar{T}_{i} T_{i}^{-1} \Pi_{i} v_{i}+T_{i}^{*} w_{i}\right)\right)_{A} .
$$

In particular, if $R_{i}=A_{i}^{-1}$, then

$$
c_{0}(v)=\inf _{\sum_{i} \Pi_{i} v_{i}=v} \sum_{i=1}^{J}\left\|P_{i} \sum_{j=i+1}^{J} \Pi_{j} v_{j}\right\|_{A_{i}}^{2},
$$

and

$$
c_{1}(v)=\inf _{\sum_{i} \Pi_{i} v_{i}=v} \sum_{i=1}^{J}\left\|P_{i} \sum_{j=i}^{J} \Pi_{j} v_{j}\right\|_{A_{i}}^{2} .
$$

Lemma 4.6. If $R_{k}=A_{k}^{-1}$ for all $k$, and $V_{k}$ are subspaces of $V$, then

$$
\frac{1}{4}\left(B_{p s c}^{-1} v, v\right) \leq\left(\bar{B}_{s s c}^{-1} v, v\right) \leq c^{*}\left(B_{p s c}^{-1} v, v\right), \quad v \in V .
$$

where

$$
c^{*}=\max _{1 \leq k \leq M}[N(k)]^{2} \text { with } N(k)=\left\{j \in\{1, \ldots, J\} \mid \text { and } V_{j} \cap V_{k} \neq\{0\}\right\} .
$$

Proof. Given $v=\sum_{i=1}^{J} v_{i}$, with $v_{i} \in V_{i}$. It follows that

$$
\|v\|_{A}^{2}=\sum_{k, j=1}^{J}\left(v_{k}, v_{j}\right)_{A}=\sum_{k=1}^{J}\left(v_{k}, v_{k}\right)_{A}+2 \sum_{j>k}^{J}\left(v_{k}, v_{j}\right)_{A}=-\sum_{k=1}^{J}\left(v_{k}, v_{k}\right)_{A}+2 \sum_{j \geq k}^{J}\left(v_{k}, v_{j}\right)_{A} .
$$


Thus

$$
\begin{aligned}
\sum_{k=1}^{J}\left\|v_{k}\right\|_{A}^{2} & \leq 2 \sum_{k=1}^{J}\left(v_{k}, \sum_{j=k}^{J} v_{j}\right)_{A}=2 \sum_{k=1}^{J}\left(v_{k}, P_{k} \sum_{j=k}^{J} v_{j}\right)_{A} \\
& \leq 2\left(\sum_{k=1}^{J}\left\|P_{k} \sum_{j=k}^{J} v_{j}\right\|_{A}^{2}\right)^{1 / 2}\left(\sum_{k=1}^{J}\left\|v_{k}\right\|_{A}^{2}\right)^{1 / 2} .
\end{aligned}
$$

Consequently

$$
\sum_{k=1}^{J}\left\|v_{k}\right\|_{A}^{2} \leq 4 \sum_{k=1}^{J}\left\|P_{k} \sum_{j=k}^{J} v_{j}\right\|_{A}^{2} .
$$

By (4.20, 4.27) and 4.25), we have

$$
\left(B_{p s c}^{-1} v, v\right) \leq 4 c_{1}(v)=4\left(\bar{B}_{s s c}^{-1} v, v\right)
$$

The upper bound also follows easily. From $\left\|P_{k}\right\|_{A}=1$ and the Schwarz inequality, we obtain

$$
\begin{aligned}
\sum_{k=1}^{J}\left\|P_{k} \sum_{j=k}^{J} v_{j}\right\|_{A}^{2} & =\sum_{k=1}^{J}\left\|P_{k} \sum_{j \in N(k) ; j \geq k} v_{j}\right\|_{A}^{2} \\
& \leq \sum_{k=1}^{J}\left\|\sum_{j \in N(k) ; j \geq k} v_{j}\right\|_{A}^{2} \leq \sum_{k=1}^{J} N(k) \sum_{j \in N(k) ; j \geq k}\left\|v_{j}\right\|_{A}^{2} \\
& \leq \sqrt{c^{*}} \sum_{k=1}^{J} \sum_{j \in N(k) ; j \geq k}\left\|v_{j}\right\|_{A}^{2} \leq c^{*} \sum_{k=1}^{J}\left\|v_{k}\right\|_{A}^{2} .
\end{aligned}
$$

The proof is concluded by taking the infimum over all decompositions on both sides and applying 4.27).

Remark 4.7. We would like to point that the estimate in (4.28) holds for anisotropic and jump coefficient problems, and the constant $c^{*}$ only depends on the "topology" of the overlaps between the subspaces and does not depend on other ingredients and properties.

The Jacobi and Gauss-Seidel method can be interpreted as PSC and SSC based on the decomposition

$$
\mathbb{R}^{n}=\sum_{i=1}^{n} \operatorname{span}\left\{e_{i}\right\}
$$

with exact subspace solves such that

$$
B_{p s c}=D^{-1}, \quad B_{s s c}=(D+L)^{-1}, \quad \bar{B}_{s s c}=(D+U)^{-1} D(D+L)^{-1} .
$$


By Theorem 4.5

$$
c_{0}=\sup _{\|v\|_{A}=1}\left(D^{-1} U v, U v\right), \quad c_{1}=\sup _{\|v\|_{A}=1}\left(D^{-1}(D+U) v,(D+U) v\right) .
$$

By Lemma 4.6

$$
\frac{1}{4}(D v, v) \leq\left(D^{-1}(D+U) v,(D+U) v\right) \leq c_{*}(D v, v), \quad v \in \mathbb{R}^{n}
$$

We note that

$$
c_{1}=\sup _{v \in V} \frac{\left(\bar{B}_{s s c}^{-1} v, v\right)}{\|v\|_{A}^{2}} \leq \sigma \sup _{v \in V} \frac{\|v\|_{D}^{2}}{\|v\|_{A}^{2}}
$$

where

$$
\sigma=\sup _{v \in V} \frac{\|v\|_{\bar{B}_{s s c}^{-1}}^{2}}{(D v, v)},
$$

In the above presentation, most results are for SPD problems. We would like to point out that all of these results can be extended a more general class of problems: namely symmetric, semi-positive definite problems. When $A$ is a matrix, we further assume that all the diagonals of $A$ are non-zero and hence positive. When the method of subspace correction is used for a more general symmetric, semipositive definite operator $A$, we further assume that each $A_{i}$ is SPD. We should use the acronym SSPD to denote matrices or operators that satisfy the aforementioned properties.

\subsection{Bibliographical notes}

The general notion of subspace corrections by means of space decompositions was described in Xu (Xu 1992) based on (Bramble, Pasciak, Wang and Xu 1991c, Bramble, Pasciak, Wang and Xu 1991b). It is an abstract point of view encompassing the theory and practice of a large class of iterative algorithms such as multigrid and domain decomposition methods. In the last two decades a great deal of effort has been put into the investigation of the theoretical and practical issues related to these methods. General results, applicable in many cases, in the theory of additive and multiplicative methods in Hilbert space is found in (Xu and Zikatanov 2002). For a literature review and basic results we refer the reader to some monographs and survey articles: (Hackbusch 1985), (Bramble 1993), (Vassilevski 2008a), (Xu 1989, Xu 1997), (Xu and Zou 1998),(Yserentant 1993), (Toselli and Widlund 2005), (Griebel and Oswald 1995), (Smith, Bjørstad and Gropp 1996). For detailed studies of classical iterative methods, we refer to the monographs (Young 1971), (Hackbusch 1994), (Varga 2000), (Saad 2003a).

We note that in this section we have considered SSPD matrices, and according to (Lee, Wu, Xu and Zikatanov 2008, Lee, Wu, Xu and Zikatanov 2007, Ayuso de Dios, Brezzi, Marini, Xu and Zikatanov 2014) all the results in this section are valid 
for SSPD problems with the semi-norm. Relations between auxiliary space method and the subspace correction methods is drawn in (Chen 2011). In the classical multigrid literature (Brandt, McCormick and Ruge 1982b, Brandt, McCormick and Ruge 1985, Ruge and Stüben 1987, Trottenberg, Oosterlee and Schüller 2001) the notions of algebraically smooth (low) frequencies and algebraic high frequencies play an important role. They are also instrumental in the design of new AMG methods. As indicated by the convergence estimates, for a given a smoother, the desirable coarse spaces should capture or approximate well the lower end of the spectrum of the relaxed matrix $\bar{R} A$ or $D^{-1} A$. This is usually referred to as nearnull space (Treister and Yavneh 2015, Lai and Olson 2011, Xu 2009), (Brezina, Falgout, MacLachlan, Manteuffel, McCormick and Ruge 2006a).

\section{Abstract multigrid methods and 2-level theory}

In this section, we will present algebraic multigrid methods in an abstract setting. The acronym "AMG" for Algebraic Multi-Grid can also be used to stand for Abstract Multi-Grid.

Our focus will be on two level methods. In view of algorithmic design, the extension of two-level to multi-level is straightforward: a general multilevel Vcycle algorithm can be obtained by recursively applying a two-level algorithm. But the extension of a two-level convergence theory to multi-level case can be highly nontrivial.

We will only consider SSPD problems as described in $\$ 4$. As is done in most literature, the designing principle of an AMG is to optimize the choice of coarse space with a given a smoother. The most commonly used smoothers are the GaussSeidel method and (modified or scaled) Jacobi method. As these smoothers are qualitatively convergent as an iterative method itself, the resulting AMG method is always qualitatively convergent. The task of our AMG convergence theory is to make sure such a convergence is also quantitatively fast. In particular, for system arising from the discretization of partial differential equations, we hope that our AMG method converges uniformly with respect to the size of the problem and/or some crucial parameters from the underlying PDEs. We sometimes call such a convergence "uniformly convergent" or "uniform convergence".

As it turns out, we are often able to establish such a uniform convergence for two-level AMG, but very rarely we can extend such a uniform convergence result to multi-level case. For second order elliptic boundary value problems, multilevel convergence are very well-understood for geometric multigrid methods. But a rigorous multilevel convergence theory for an AMG without using geometric information is still a widely open problem.

We will mainly focus on two-level convergence theory on AMG methods in this section and also in the rest of this paper. 


\subsection{A two-level method}

A two-level method typically consists of the following components:

1. A smoother $R: V^{\prime} \mapsto V$;

2. A coarse space $V_{c}$, which may or may not be a subspace of $V$ but linked with $V$ via a prolongation operator:

$$
P: V_{c} \mapsto V
$$

3. A coarse space solver $B_{c}: V_{c}^{\prime} \mapsto V_{c}$.

In the discussion below we need the following inner product

$$
(u, v)_{\bar{R}^{-1}}=\left(\bar{T}^{-1} u, v\right)_{A}=\left(\bar{R}^{-1} u, v\right), \quad \bar{T}=\bar{R} A,
$$

and the accompanying norm $\|\cdot\|_{\bar{R}^{-1}}$. Here we recall that the definition of $\bar{R}$ is analogous to that in (4.5).

We always assume that $\bar{R}$ is SPD and hence the smoother $R$ is always convergent. Further more,

$$
\|v\|_{A}^{2} \leq\|v\|_{\bar{R}^{-1}}^{2} .
$$

The restriction of (4.1) is then

$$
A_{c} u_{c}=f_{c},
$$

where

$$
A_{c}=P^{\prime} A P, \quad f_{c}=P^{\prime} f .
$$

The coarse space solver $B_{c}$ is often chosen to be the exact solver, namely $B_{c}=$ $A_{c}^{-1}$, for analysis, but in a multilevel setting, $B_{c}$ is recursively defined and it is an approximation for $A_{c}^{-1}$. We distinguish these two different cases in choosing $B_{c}$ :

$$
\begin{aligned}
& \text { exact two level method if } B_{c}=A_{c}^{-1} . \\
& \text { inexact two level method if } B_{c} \neq A_{c}^{-1} .
\end{aligned}
$$

In the case that $A$ is semi-definite, we use $N$ to denote the kernel of $A$ and we always assume that $N \subset V_{c}$. Let

$$
W:=N^{\perp} \text {, and } W_{c}:=V_{c} \cap W,
$$

where the orthogonality is understood with respect to the $(\cdot, \cdot)_{\bar{R}^{-1}}$ inner product. Let $Q_{1}: V \mapsto W$ be the orthogonal projection respect to $(\cdot, \cdot)_{\bar{R}^{-1}}$ inner product

$$
\left(Q_{1} v, w\right)_{\bar{R}^{-1}}=(v, w)_{\bar{R}^{-1}}, \quad \text { for all } \quad v \in V, w \in W .
$$

$A_{c}$ is semi-definite on $V_{c}$ but invertible on $W_{c}$. We denote the restriction of $A_{c}$ on $W_{c}$ by $\hat{A}_{c}$, and define the psudo-inverse of $A_{c}$

$$
A_{c}^{\dagger}:=Q_{1}^{\prime} \hat{A}_{c}^{-1} Q_{1} \text {. }
$$


With a slight abuse of notation, we will still use $A_{c}^{-1}$ to denote the psudo-inverse of $A_{c}$, namely

$$
A_{c}^{-1}=A_{c}^{\dagger} .
$$

We will use similar notation for psudo-inverse of other relevant singular operators and matrices in the rest of the paper.

We choose to define an AMG algorithm in terms of an operator $B: V^{\prime} \mapsto V$, which can be considered as an approximate inverse or a preconditioner of $A$. A typical two level MG method is as follows.

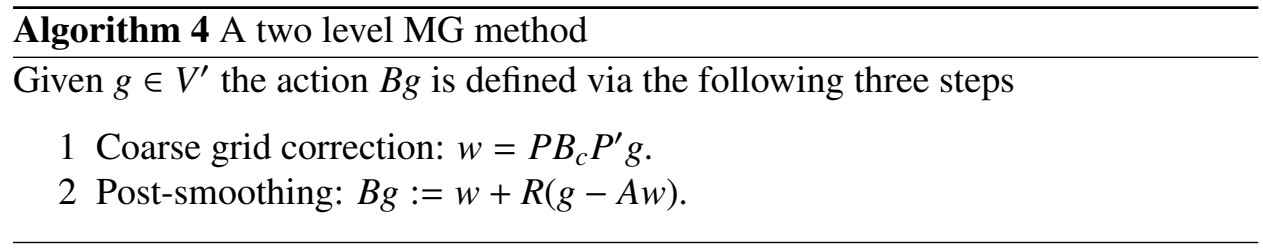

In the rest of this section, we take $B_{c}=A_{c}^{-1}$.

There are usually two different (and mathematically equivalent) ways to choose $V_{c}, V, P$ and $R$. The first one, known as operator version, is such that

$$
V_{c} \subset V .
$$

In this case, $P=\imath_{c}$ where

$$
l_{c}: V_{c} \rightarrow V,
$$

is the natural inclusion of $V_{c}$ into $V$. In the application to finite element discretization for 2nd order elliptic boundary value problems, $V_{c}$ and $V$ are just the finite element subspaces of $H^{1}(\Omega)$. This type of notation is convenient for analysis. But this is not the algorithm that can be directly used for implementation.

The second one, known as matrix version, is such that

$$
V_{c}=\mathbb{R}^{n_{c}} \text { and } V=\mathbb{R}^{n},
$$

and

$$
P: \mathbb{R}^{n_{c}} \mapsto \mathbb{R}^{n},
$$

is the prolongation matrix.

These two different set of notations are related through the use of basis functions of $\left\{\phi_{i}^{c}\right\}_{i=1}^{n_{c}} \subset V_{c}$ and $\left\{\phi_{i}:\right\}_{i=1}^{n} \subset V$. As noted earlier, the prolongation matrix $P$ given in (3.12) - (3.13) is simply the matrix representation of $t_{c}$ given in (5.9), and we have

$$
\left(\phi_{i}^{c}, \ldots, \phi_{n_{c}}^{c}\right)=\left(\phi_{i}, \ldots, \phi_{n}\right) P .
$$

The following observation is clear. 
Observation 5.12. Finding a coarse space $V_{c} \subset V$ is equivalent to finding a prolongation matrix $P$ in 5.10 .

Lemma 5.1. The error propagation operator for two-level AMG operator $E=$ $I-B A$ is

$$
E=(I-R A)\left(I-\Pi_{c}\right),
$$

where $\Pi_{c}=\imath_{c} A_{c}^{-1} \imath_{c}^{\prime} A$, which is the $(\cdot, \cdot)_{A}$ orthogonal projection on $V_{c}$, in matrix notation $\Pi_{c}=P A_{c}^{-1} P^{T} A$.

\subsection{An optimal two-level AMG theory}

The design of AMG method is to balance the interplay between smoother $R$ and the coarse space $V_{c}$. The design of most existing AMG is to first fix a smoother, which is often given by Jacobi or Gauss-Seidel method (or their combinations and variations), and then to optimize the choice of coarse space. This is the approach that we discuss mostly in this paper. But we also comment on a different approach by first fixing the coarse space and then trying to optimize the choice of the smoother. It is also possible to try to make optimal choice of smoother and coarse space simultaneously, but we will not address this approach in this paper.

Let $Q_{c}: V \mapsto V_{c}$ be orthogonal projection respect to $(\cdot, \cdot)_{\bar{R}^{-1}}$ inner product

$$
\left(Q_{c} u, v_{c}\right)_{\bar{R}^{-1}}=\left(u, v_{c}\right)_{\bar{R}^{-1}}, \quad \text { for all } \quad v_{c} \in V_{c} .
$$

By the definition of $W$ and $W_{c}$ we have that $(\cdot, \cdot)_{A}$ is an inner product on $W,\|\cdot\|_{A}$ is a norm on $W$, and the projection $\Pi_{c}: V \mapsto W_{c}$ is well defined:

$$
\left(\Pi_{c} u, v_{c}\right)_{A}=\left(u, v_{c}\right)_{A}, \quad \forall u \in V, v_{c} \in W_{c} .
$$

The two level convergence rate is obtained in the following theorem.

Theorem 5.2. Assume that $N \subset V_{c}$. The convergence rate of an exact two level $\mathrm{AMG}$ is given by

$$
\|E\|_{A}^{2}=1-\frac{1}{K\left(V_{c}\right)}
$$

where

$$
K\left(V_{c}\right)=\max _{v \in W} \frac{\left\|\left(I-Q_{c}\right) v\right\|_{\bar{R}^{-1}}^{2}}{\|v\|_{A}^{2}}=\max _{v \in W} \min _{v_{c} \in W_{c}} \frac{\left\|v-v_{c}\right\|_{\bar{R}^{-1}}^{2}}{\|v\|_{A}^{2}} .
$$

Proof. We notice that.

$$
\|(I-T) v\|_{A}^{2}=((I-\bar{T}) v, v)_{A}, \forall v \in V .
$$


Then we have

$$
\begin{aligned}
\|E\|_{A}^{2} & =\max _{w \in W} \frac{\left\|(I-T)\left(I-\Pi_{c}\right) w\right\|_{A}^{2}}{\|w\|_{A}^{2}} \\
& =\max _{w \in W} \frac{\left((I-\bar{T})\left(I-\Pi_{c}\right) w,\left(I-\Pi_{c}\right) w\right)_{A}}{\|w\|_{A}^{2}} \\
& =1-\min _{w \in W} \frac{\left(\bar{T}\left(I-\Pi_{c}\right) w,\left(I-\Pi_{c}\right) w\right)_{A}}{\|w\|_{A}^{2}} \\
& =1-\min _{w \in W} \frac{\left(Q_{1} \bar{T}\left(I-\Pi_{c}\right) w,\left(I-\Pi_{c}\right) w\right)_{A}}{\left\|\left(I-\Pi_{c}\right) w\right\|_{A}^{2}+\left\|\Pi_{c} w\right\|_{A}^{2}} \\
& =1-\min _{v \in W_{c}^{\perp_{A}}} \frac{\left(Q_{1} \bar{T} v, v\right)_{A}}{\|v\|_{A}^{2}} \\
& =1-\min _{v \in W_{c}^{\perp_{A}}} \frac{\left(\left(I-\Pi_{c}\right) Q_{1} \bar{T} v, v\right)_{A}}{\|v\|_{A}^{2}} \\
& =1-\lambda_{\min }(X),
\end{aligned}
$$

where

$$
X=\left(I-\Pi_{c}\right) Q_{1} \bar{T}: W_{c}^{\perp_{A}} \mapsto W_{c}^{\perp_{A}},
$$

and it is easy to see that $X$ is self-adjoint with respect to $(\cdot, \cdot)_{A}$.

One key observation is that the inverse of $X$ can be explicitly written as

$$
Z=\left(Q_{1} \bar{T}\right)^{-1}\left(I-Q_{c}\right),
$$

since by definition, we have, for any $u, v \in V$

$$
\begin{aligned}
\left(\Pi_{c} Z u, v\right)_{A} & =\left(\left(Q_{1} \bar{T}\right)^{-1}\left(I-Q_{c}\right) u, \Pi_{c} v\right)_{A}=\left(\bar{T}\left(Q_{1} \bar{T}\right)^{-1}\left(I-Q_{c}\right) u, \Pi_{c} v\right)_{\bar{R}^{-1}} \\
& =\left(Q_{1} \bar{T}\left(Q_{1} \bar{T}\right)^{-1}\left(I-Q_{c}\right) u, \Pi_{c} v\right)_{\bar{R}^{-1}}=\left(\left(I-Q_{c}\right) u, \Pi_{c} v\right)_{\bar{R}^{-1}}=0
\end{aligned}
$$

which implies $\Pi_{c} Z=0$. Thus we have

$$
Z: W_{c}^{\perp_{A}} \mapsto W_{c}^{\perp_{A}},
$$

and furthermore

$$
X Z=\left(I-\Pi_{c}\right)\left(I-Q_{c}\right)=I-\Pi_{c}=I \text { on } W_{c}^{\perp_{A}} .
$$


Consequently $\lambda_{\min }(X)=\frac{1}{\lambda_{\max }(Z)}$. Finally,

$$
\begin{aligned}
\lambda_{\max }(Z) & =\max _{v \in W_{c}^{\perp_{A}}} \frac{\left(\left(Q_{1} \bar{T}\right)^{-1}\left(I-Q_{c}\right) v, v\right)_{A}}{(v, v)_{A}}=\max _{v \in W_{c}^{\perp_{A}}} \frac{\left(\bar{T}\left(Q_{1} \bar{T}\right)^{-1}\left(I-Q_{c}\right) v, v\right)_{\bar{R}^{-1}}}{(v, v)_{A}} \\
& =\max _{v \in W_{c}^{\perp_{A}}} \frac{\left(Q_{1} \bar{T}\left(Q_{1} \bar{T}\right)^{-1}\left(I-Q_{c}\right) v, v\right)_{\bar{R}^{-1}}}{(v, v)_{A}}=\max _{v \in W_{c}^{\perp_{A}}} \frac{\left(\left(I-Q_{c}\right) v, v\right)_{\bar{R}^{-1}}}{(v, v)_{A}} \\
& =\max _{v \in W_{c}^{\perp_{A}}} \frac{\left\|\left(I-Q_{c}\right) v\right\|_{\bar{R}^{-1}}^{2}}{(v, v)_{A}}=K\left(V_{c}\right) .
\end{aligned}
$$

The last identity holds because $I-Q_{c}=\left(I-Q_{c}\right)\left(I-\Pi_{c}\right)$ and we can then take the maximum over all $v \in W$. This completes the proof. $\square$

Theorem 5.2 can be stated as follows using the matrix representation introduced in $\$ 3$.

Theorem 5.3. Assume that $P \in \mathbb{R}^{n \times n_{c}}$ and $N(\tilde{A}) \subset$ Range $(P)$. The convergence rate of an exact two level AMG is given by

$$
\|E\|_{A}^{2}=1-\frac{1}{\tilde{K}(P)}
$$

where

$$
\tilde{K}(P)=\max _{v \in \mathbb{R}^{n}} \min _{v_{c} \in \mathbb{R}^{n_{c}}} \frac{\left\|v-P v_{c}\right\|_{\tilde{\tilde{R}}^{-1}}^{2}}{\|v\|_{\tilde{A}}^{2}} .
$$

Remark 5.4. The result in the theorem above can be viewed as follows. We note that $\bar{T}^{-1}\left(I-Q_{c}\right)$ is a selfadjoint operator in $A$ inner product. Hence, we immediately have

$$
K\left(V_{c}\right)=\left\|\bar{T}^{-1}\left(I-Q_{c}\right)\right\|_{A}=\left\|(\bar{R} A)^{-1}\left(I-Q_{c}\right)\right\|_{A} .
$$

In case of two subspaces, we have the following theorem giving the precise convergence rate of the corresponding SSC method.

Theorem 5.5. Let $\left\{\mu_{j}, \zeta_{j}\right\}_{j=1}^{n}$ be the eigenpairs of $\bar{T}=\bar{R} A$. And let assume that $\left\{\zeta_{j}\right\}$ are orthogonal with respect to $(\cdot, \cdot)_{\bar{R}^{-1}}$. The convergence rate $\left\|E\left(V_{c}\right)\right\|_{A}$ is minimal for coarse space

$$
V_{c}^{\mathrm{opt}}=\operatorname{span}\left\{\zeta_{j}\right\}_{j=1}^{n_{c}} \in \underset{\operatorname{dim} V_{c}=n_{c}, N \subset V_{c}}{\arg \min } K\left(V_{c}\right) .
$$

In this case,

$$
\|E\|_{A}^{2}=1-\mu_{n_{c}+1} .
$$

Proof. By Theorem 5.2, we just need to maximize $\frac{1}{K\left(V_{c}\right)}$. For any $v \in V_{c}^{\perp}$, where $\perp$ is with respect to the $\bar{R}^{-1}$ inner product, we have

$$
\min _{v_{c} \in V_{c}}\left\|v-v_{c}\right\|_{\bar{R}^{-1}}^{2}=\|v\|_{\bar{R}^{-1}}^{2} \text {. }
$$


Then it follows that

$$
\frac{1}{K\left(V_{c}\right)}=\min _{v \in V} \max _{v_{c} \in V_{c}} \frac{\|v\|_{A}^{2}}{\left\|v-v_{c}\right\|_{\bar{R}^{-1}}^{2}} \leq \min _{v \in V_{c}^{\perp} \max _{c} \in V_{c}} \frac{\|v\|_{A}^{2}}{\left\|v-v_{c}\right\|_{\bar{R}^{-1}}^{2}}=\min _{v \in V_{c}^{\perp}} \frac{\|v\|_{A}^{2}}{\|v\|_{\bar{R}^{-1}}^{2}} .
$$

By the min-max principle (Theorem 2.1), we have

$$
\max _{\operatorname{dim} V_{c}=n_{c}} \frac{1}{K\left(V_{c}\right)} \leq \max _{\operatorname{dim} V_{c}=n_{c}} \min _{v \in V_{c}^{\perp}} \frac{\|v\|_{A}^{2}}{\|v\|_{\bar{R}^{-1}}^{2}}=\mu_{n_{c}+1} .
$$

On the other hand, if we choose $V_{c}^{\mathrm{opt}}=\operatorname{span}\left\{\zeta_{j}\right\}_{j=1}^{n_{c}}$, it is easy to compute that $K\left(V_{c}^{\mathrm{opt}}\right)=\frac{1}{\mu_{n_{c}+1}}$. So we have

$$
\max _{\operatorname{dim} V_{c}=n_{c}} \frac{1}{K\left(V_{c}\right)}=\mu_{n_{c}+1},
$$

with optimal coarse space

$$
V_{c}^{\mathrm{opt}}=\operatorname{span}\left\{\zeta_{j}\right\}_{j=1}^{n_{c}}
$$

Using the matrix representation introduced in $\$ 3$, we state the matrix version of Theorem 5.5 below. For simplicity, with an abuse of notation, we still use $A$ to denote the matrix representation of operator $A$.

Theorem 5.6. Let $\left\{\mu_{j}, \zeta_{j}\right\}_{j=1}^{n}$ be the eigenpairs of $\bar{T}=\bar{R} A$. And let assume that $\left\{\zeta_{j}\right\}$ are orthogonal with respect to $(\cdot, \cdot)_{\bar{R}^{-1}}$. The convergence rate $\|E(P)\|_{A}$ is minimal for $P$ such that

$$
\operatorname{Range}(P)=\operatorname{Range}\left(P^{\text {opt }}\right)
$$

where

$$
P^{\mathrm{opt}}=\left(\zeta_{1}, \ldots \zeta_{n_{c}}\right)
$$

In this case,

$$
\|E\|_{A}^{2}=1-\mu_{n_{c}+1}
$$

The following theorem is important in motivating most AMG algorithms.

Theorem 5.7. Given $\eta>0$, let $\mathcal{X}_{\eta}$ be defined as

$$
X_{\eta}=\left\{P \in \mathbb{R}^{n \times n_{c}}:(P v, P v)_{\bar{R}^{-1}} \geq \eta(v, v), \quad v \in \mathbb{R}^{n_{c}}\right\},
$$

Then, with $P^{\text {opt }}$ given by (5.24), we have $P \in \arg \min _{Q \in \mathcal{X}_{\eta}} \operatorname{trace}\left(Q^{T} A Q\right)$ if

$$
\left.P \in \mathcal{X}_{\eta} \text { and } \operatorname{Range}(P)=\operatorname{Range}\left(P^{\text {opt }}\right)\right) \text {. }
$$

Since the eigenvalues of $\bar{R} A$ are expensive to compute, the practical value of Theorem 5.5 is limited. But it provides useful guidance in the design practical AMG method. 


\subsection{Quasi-optimal theories}

For finite element discretizations we can use the Weyl's Law combined with Theorem 5.5 to prove an estimate on the convergence rate of a two-grid method with optimal coarse space.

Corollary 5.8. Let the assumptions of the discrete Weyl's law (Theorem 2.5) hold and the smoother $\bar{R}$ be spectrally equivalent to the diagonal of the stiffness matrix $A$. Let $\gamma>0$ be such that $\gamma n \leq n_{c}<n$. Then, for the optimal coarse space, we have the estimate,

$$
\mu_{n_{c}+1} \geq \delta_{0}, \quad \text { and, } \quad\|E\|_{A}^{2} \leq 1-\delta_{0} .
$$

where $\delta_{0} \in(0,1)$ only depends on $\gamma$ and the constants $\gamma_{0}$ and $\gamma_{1}$ in 2.31).

Proof. By the assumptions in Theorem 2.5 and the fact that $\bar{R}$ is spectrally equivalent to the diagonal of $A$ we have

$$
|w|_{1}^{2} \equiv\|w\|_{A}^{2}, \quad\|w\|_{0}^{2} \equiv h^{2}\|w\|_{\bar{R}^{-1}}^{2} .
$$

Further, Lemma 2.4 and Theorem 2.5 then show that

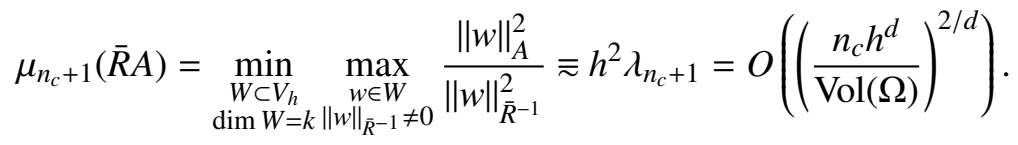

The desired result follows immediately from Theorem 5.5 because $\operatorname{Vol}(\Omega) \equiv h^{d} n$ and $\gamma n \leq n_{c}<n$, which gives $\mu_{n_{c}+1} \equiv 1$.

Remark 5.9. Since the coarse space which minimizes the convergence rate is the coarse space which minimizes also $K\left(V_{c}\right)$ and as a corollary we have the following equality

$$
K\left(V_{c}\right)=\frac{1}{1-\|E\|_{A}^{2}} \geq \frac{1}{\mu_{n_{c}+1}}
$$

or

$$
\|E\|_{A}^{2} \geq 1-\mu_{n_{c}+1} .
$$

Theorem 5.2 provides an explicit estimate on the convergence of a two level method in terms of $K\left(V_{c}\right)$. For a given method, a smaller bound on $K\left(V_{c}\right)$ means a faster convergence rate. In particular, the two-level AMG method is uniformly convergent if $K\left(V_{c}\right)$ is uniformly bounded with respect to mesh parameters.

\subsection{Quasi-optimal theories}

We now look at the necessary and sufficient condition for uniform convergence of a two level method as proved in $\$ 5.2$ (see Theorem 5.2):

$$
\min _{v_{c} \in V_{c}}\left\|v-v_{c}\right\|_{\bar{R}^{-1}} \leq K\left(V_{c}\right)\|v\|_{A}^{2} .
$$


Here $K\left(V_{c}\right)$ is the smallest constant for which 5.29 holds for all $v \in V$. The space $V_{c}$ which minimizes $K\left(V_{c}\right)$ is $V_{c}^{\text {opt }}$. Similar argument was used in the proof of Theorem 5.5 in $\$ 5.2$. We generalize here the result to semidefinite $A$.

For a given smoother $R$, one basic strategy in the design of AMG is to find a coarse space such that $K\left(V_{c}\right)$ is made as practically small as possible. There are many cases, however, in which the operator $\bar{R}^{-1}$ in the definition of $K\left(V_{c}\right)$ is difficult to work with.

One commonly used approach is to replace $\bar{R}^{-1}$ by a simpler but spectrally equivalent SPD operator. More specifically, we assume that $D: V \mapsto V^{\prime}$ is an SPD operator such that

$$
c_{D}(D v, v) \leq\left(\bar{R}^{-1} v, v\right) \leq c^{D}(D v, v), \quad \forall v \in V
$$

Namely

$$
c_{D}\|v\|_{D}^{2} \leq\|v\|_{\bar{R}^{-1}}^{2} \leq c^{D}\|v\|_{D}^{2}, \quad \forall v \in V
$$

where

$$
(u, v)_{D}=(D u, v), \quad\|v\|_{D}^{2}=(v, v)_{D} .
$$

Example of such equivalent norms for Schwarz smoothers are given in 4.20) and (4.27). As a rule, the norm defined by $\bar{R}$ corresponding to the symmetric Gauss-Seidel method, i.e. $R$ defined by pointwise Gauss-Seidel method can be replaced by the norm defined by the diagonal of $A$ (i.e. by Jacobi method, which, while not always convergent as a relaxation provides an equivalent norm).

In terms of this operator $D$, we introduce the following quantity

$$
K\left(V_{c}, D\right)=\max _{v} \frac{\left\|v-Q_{D} v\right\|_{D}^{2}}{\|v\|_{A}^{2}}=\max _{v} \min _{v_{c} \in V_{c}} \frac{\left\|v-v_{c}\right\|_{D}^{2}}{\|v\|_{A}^{2}},
$$

where $Q_{D}: V \mapsto V_{c}$ is the $(u, v)_{D}$-orthogonal projection.

By (5.17), 5.32) and (5.31), we have

$$
c_{D} K\left(V_{c}, D\right) \leq K\left(V_{c}\right) \leq c^{D} K\left(V_{c}, D\right) .
$$

Theorem 5.10. The two level algorithm satisfies

$$
1-\frac{1}{c_{D} K\left(V_{c}, D\right)} \leq\|E\|_{A}^{2} \leq 1-\frac{1}{c^{D} K\left(V_{c}, D\right)} \leq 1-\frac{1}{c^{D} C} .
$$

where $C$ is any upper bound of $K\left(V_{c}, D\right)$, namely

$$
\min _{w \in V_{c}}\|v-w\|_{D}^{2} \leq C\|v\|_{A}^{2}, \quad \text { for all } \quad v \in V .
$$

The proof of the above theorem is straightforward and indicates that, if $c_{D}$ and $c^{D}$ are "uniform" constants, the convergence rate of the two-level method is "uniformly" dictated by the quantity $K\left(V_{c}, D\right)$.

We say that $V_{c}$ is quasi-optimal if the following inequality holds

$$
\min _{w \in V_{c}}\|v-w\|_{D}^{2} \leq \gamma \mu_{n_{c}+1}^{-1}\|v\|_{A}^{2}, \quad \text { for all } v \in V,
$$


with a constant $\gamma>0$ independent of the size of the problem.

The construction of an approximation to the optimal coarse space $V_{c}^{\mathrm{opt}}$ which is used in most AMG algorithms relies on two operators $A_{M}$ and $D_{M}$ which satisfy:

$$
c_{1}\|v\|_{D}^{2} \leq\|v\|_{D_{M}}^{2}, \quad\|v\|_{A_{M}}^{2} \leq c_{2}\|v\|_{A}^{2}, \quad \text { for all } \quad v \in V
$$

with constants $c_{1}$ and $c_{2}$ independent of the problem size. Here, on the right side, we have a seminorm $\|\cdot\|_{A_{M}}$, because sometimes $A_{M}$ is only semi-definite. We point out that here $A_{M}$ and $D_{M}$ are analogues to $A_{W}$ and $\underline{D}$ defined in 6.6) and (6.7), respectively, in the general framework in $\$ 6$. And the assumptions in (5.37) are analogous to the Assumptions 6.9 which we made in the general AMG framework in $\$ 6$.

Theorem 5.11. If $D_{M}$ and $A_{M}$ satisfy (5.37), and $V_{c}$ is a coarse space such that

$$
\min _{w \in V_{c}}\|v-w\|_{D_{M}}^{2} \leq \gamma \mu_{n_{c}+1}^{-1}\|v\|_{A_{M}}^{2}, \quad \text { for all } v \in V \text {, }
$$

Then

1 The following estimate holds

$$
\min _{w \in V_{c}}\|v-w\|_{\bar{R}^{-1}}^{2} \leq \frac{c^{D}}{c_{D}} \frac{c_{2}}{c_{1}} \gamma \mu_{n_{c}+1}^{-1}\|v\|_{A}^{2}, \quad \text { for all } v \in V .
$$

2 The corresponding two-level AMG algorithm satisfies

$$
\|I-B A\|_{A}^{2} \leq 1-\frac{c_{D}}{c^{D}} \frac{c_{1}}{c_{2}} \frac{1}{\gamma} \mu_{n_{c}+1}
$$

\subsection{Algebraically high and low frequencies}

In geometric MG, algebraically smooth error is also smooth in the usual geometric sense. However, in AMG settings, smooth error can be geometrically non-smooth. In order to make this distinction, we use the term algebraically smooth error when we refer to the error in the AMG setting that is not damped (eliminated) by the smoother $R$. In general, a good interpretation of the algebraically smooth error leads to an efficient and robust AMG algorithm. A careful characterization of the algebraically smooth error is needed, since in such case we can try to construct a coarser level which captures these error components well.

Here is a more formal definition of a algebraically smooth error.

Definition 5.12. Let $R: V \mapsto V$ be a smoothing operator such that its symmetrization $\bar{R}=R+R^{T}-R^{T} A R$ is positive definite. Given $\varepsilon \in(0,1)$, we say that the vector $v$ is algebraically $\varepsilon$-smooth (or $v$ is an $\varepsilon$-algebraic low frequency) with respect to $A$ if

$$
\|v\|_{A}^{2} \leq \varepsilon\|v\|_{\bar{R}^{-1}}^{2} .
$$

The set of algebraically smooth vectors will be denoted by:

$$
\mathcal{L}_{\varepsilon}=\left\{v:\|v\|_{A}^{2} \leq \varepsilon\|v\|_{\bar{R}^{-1}}^{2}\right\} .
$$


We point out that this is a set of vectors (a ball, or, rather, an ellipsoid) and not a linear vector space in general. It is then clear that the elements of this set need to be approximated well by elements from the coarse space.

The rationale of the above definition can be seen from the following simple result (We notice it is always true that $\left.(\bar{R} A v, v) \leq\|v\|_{A}^{2}\right)$.

Lemma 5.13. Any vector $v \in V$ that satisfies

$$
(\bar{R} A \boldsymbol{v}, \boldsymbol{v})_{A} \leq \varepsilon\|v\|_{A}^{2} .
$$

is $\varepsilon$-algebraically smooth.

Proof. By the Schwarz inequality for the inner product defined by $\bar{R}^{-1}$ and 5.43 we have

$$
\|\boldsymbol{v}\|_{A}^{2}=\left(\bar{R} A \boldsymbol{v}, \bar{R}^{-1} \boldsymbol{v}\right) \leq(\bar{R} A \boldsymbol{v}, A \boldsymbol{v})^{1 / 2}\left(\bar{R}^{-1} \boldsymbol{v}, \boldsymbol{v}\right)^{1 / 2} \leq \sqrt{\varepsilon}\left(\bar{R}^{-1} \boldsymbol{v}, \boldsymbol{v}\right)^{1 / 2}\|\boldsymbol{v}\|_{A}
$$

We can easily show that this definition is equivalent to saying that the algebraically smooth error components are the components for which the smoother converges slowly. Indeed, the inequality (5.43) is clearly equivalent to $((I-\bar{R} A) v, v)_{A} \geq$ $(1-\varepsilon)(v, v)_{A}$, namely,

$$
\frac{\|S \boldsymbol{v}\|_{A}^{2}}{\|\boldsymbol{v}\|_{A}^{2}} \geq 1-\varepsilon, \quad S=I-T, \quad \text { and } \quad T=R A .
$$

The property (5.44) is often referred to as smoothing property.

Remark 5.14. In the classical multigrid literature algebraically smooth error is defined as $e \in V$ such that

$$
\|e\|_{A D^{-1} A}^{2} \leq \varepsilon\|e\|_{A}^{2}
$$

for a small and positive parameter $\varepsilon$ which implies

$$
\|e\|_{A}^{2} \leq\|e\|_{D}\|e\|_{A D^{-1} A} \leq \sqrt{\varepsilon}\|e\|_{D}\|e\|_{A} .
$$

Namely,

$$
\|e\|_{A}^{2} \leq \varepsilon\|e\|_{D}^{2} .
$$

As it is clearly seen from Definition 5.12, Lemma 5.13 implies 5.46) with $\bar{R} \approx$ $D^{-1}$, where $D$ is the diagonal of $A$.

Thanks to (7.9), we have

Lemma 5.15. If $e$ is algebraically smooth, namely $e$ satisfies (5.47). Then

$$
\|e\|_{\tilde{A}}^{2} \lesssim \varepsilon\|e\|_{\tilde{D}}^{2} .
$$

Namely $e$ is also algebraically smooth with respect $\tilde{A}$, the M-matrix relative of $A$. 
On the other hand, note that by the definition of $\|\cdot\|_{\bar{R}^{-1}}$, we always have $\|v\|_{\bar{R}^{-1}} \geq$ $C\|v\|_{A}$ with constant $C$ independent of the parameters of interest. Drawing from analogy with geometric multigrid method we introduce the notion of algebraic high frequency as follows:

Definition 5.16. Given $\delta \in(0,1]$, we call $v \in V$ an $\delta$-algebraic high frequency if,

$$
\|v\|_{A}^{2} \geq \delta\|v\|_{\bar{R}^{-1}}^{2} .
$$

The set of algebraically high frequency vectors will be denoted by:

$$
\mathcal{H}_{\delta}=\left\{v:\|v\|_{A}^{2} \geq \delta\|v\|_{\bar{R}^{-1}}^{2}\right\} .
$$

The concept of algebraic high-and low-frequencies will be used in a 2-level AMG theory $\$ 5.3$ and also be used in the design of classical AMG $\$ 12.1$.

Lemma 5.17. Let $\left(\phi_{i}, \mu_{i}\right)$ are all the eigen-pairs for $\bar{R} A$, namely $\bar{R} A \phi_{i}=\mu_{i} \phi_{i}$. Then

$$
\operatorname{span}\left\{\phi_{i}: \mu_{i} \leq \varepsilon\right\} \subset \mathcal{L}_{\varepsilon}
$$

and

$$
\operatorname{span}\left\{\phi_{i}: \mu_{i} \geq \delta\right\} \subset \mathcal{H}_{\delta}
$$

We now introduce the notion of near-null space as follows.

Definition 5.18. (Near-null space) For sufficiently small $\varepsilon \in(0,1)$, we call $\operatorname{span}\left\{\phi_{i}\right.$ : $\left.\mu_{i} \leq \varepsilon\right\}$ an $\varepsilon$-near-null space of $\bar{R} A$.

\subsection{Smoothing properties of Jacobi and Gauss-Seidel methods}

The essence of multigrid methods is that simple iterative methods such as Jacobi and Gauss-Seidel methods that have a special property, known-as the smoothing property. As an illustration, we apply the Gauss-Seidel method to

$$
A \mu=b,
$$

with $A$ given by (2.16) for isotropic problem and (2.34) for anisotropic problem respectively. We first choose $\mu$ randomly (as shown in Fig. 5.2) and then compute $A \mu$ for both 2.16 and (2.34) to compute right hand sides $b=A \mu$ respectively. We then apply Gauss-Seidel method to both equations with initial guess $\mu^{0}=0$.

We note that, for $A$ given by (2.34) when $\epsilon \ll 1$, we have

$$
\lambda_{11}<\lambda_{21}<\ldots<\lambda_{N 1}<\lambda_{i j}, \quad i \geq 1, j \geq 2 .
$$

The corresponding eigen functions, which can be viewed as "algebraic low-frequencies" can be highly oscillatory in $x$-direction.

As an illustration of the difference between algebraic high/low frequencies and geometric high/low frequencies, we consider the linear system given by (2.34) for 


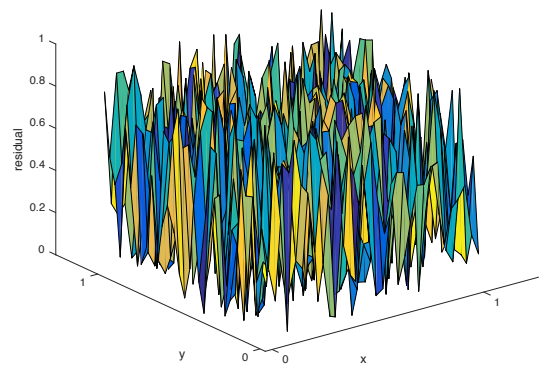

Figure 5.2. Initial error for both 2.16 and 2.34.
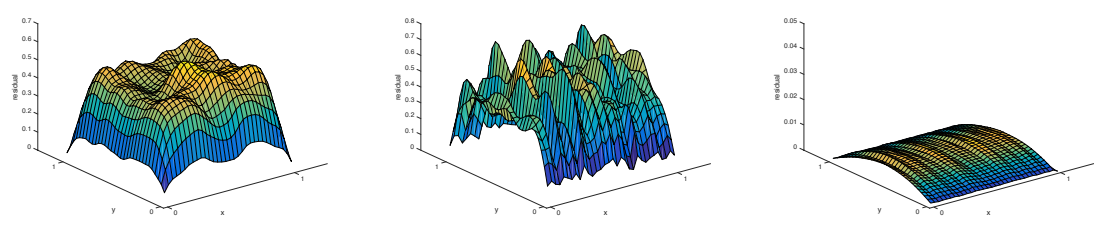

Figure 5.3. Left: error after applying 5 Gauss-Seidel iterations to 2.16. Middle: error after applying 5 Gauss-Seidel iterations to (2.34). Right: error after applying 1 block Gauss-Seidel iterations to 2.34.

anisotropic problem. Clearly, $A$ can be written as

$$
A=\epsilon I \otimes M+M \otimes I \text { with } M=\operatorname{tridiag}(-1,2,-1) .
$$

We define the vector $\mu \in R^{N}$ as

$$
\mu=x \otimes y, \text { with } x=\mathbf{1}_{n}, \text { and } y=\left(\begin{array}{llllllll}
1 & 0 & 1 & 0 & \cdots & 1 & 0 & 1
\end{array}\right)^{T} \in R^{n} .
$$

Then it is easy to compute that

$$
M x=\left(\begin{array}{c}
1 \\
0 \\
0 \\
\vdots \\
0 \\
1
\end{array}\right) \text {, and } M y=\left(\begin{array}{c}
2 \\
-2 \\
2 \\
\vdots \\
-2 \\
2
\end{array}\right) .
$$

We have

$$
A \mu=\epsilon(I \otimes M)(x \otimes y)+(M \otimes I)(x \otimes y)=\epsilon(x \otimes M y)+(M x \otimes y),
$$


and

$$
\begin{aligned}
\|\mu\|_{A}^{2} & =\mu^{T} A \mu=\epsilon(x \otimes y)^{T}(x \otimes M y)+(x \otimes y)^{T}(M x \otimes y) \\
& =\epsilon\left(x^{T} x\right) \otimes\left(y^{T} M y\right)+\left(x^{T} M x\right) \otimes\left(y^{T} y\right)=\epsilon n(n+1)+n+1 .
\end{aligned}
$$

Letting $D$ be the diagonal of $A$, we then have

$$
\|\mu\|_{D}^{2}=2(1+\epsilon) \mu^{T} \mu=(1+\epsilon) n(n+1) .
$$

This shows that

$$
\frac{\|\mu\|_{A}^{2}}{\|\mu\|_{D}^{2}}=\frac{\epsilon}{1+\epsilon}+\frac{1}{(1+\epsilon)(n+1)},
$$

which implies that $\mu$ is an algebraic low frequency if $\epsilon$ is sufficiently small.

On the other hand, if we denote the nodal basis functions corresponding to the uniform finite element mesh by $\left\{\phi_{i j}: 1 \leq i, j \leq n\right\}$, namely, $\phi_{i j}$ is a piecewise linear function such that

$$
\phi_{i j}(k h, l h)=\delta_{i k} \delta_{j l}
$$

Then we define

$$
\Phi=\left(\phi_{11}, \phi_{12}, \cdots, \phi_{1 n}, \phi_{21}, \cdots, \phi_{n n}\right) .
$$

if we consider the finite element function corresponding to $\mu$, namely, the function defined by

$$
u=\boldsymbol{\Phi} \mu=\sum_{i=1}^{\frac{n+1}{2}} \sum_{j=1}^{n} \phi_{2 i-1, j} .
$$

Then, in the geometric point of view, this function is highly oscillatory on the $x$ direction, which is a geometric high frequency (see Figure 5.4)

\subsection{Convergence theory in view of algebraic high-and low-frequencies}

We next present a convergence theory based on algebraic high- and low-frequencies. We first prove the following lemma.

Lemma 5.19. If $V_{c} \subset V$ is such that the following "stable decomposition" holds:

$$
V=V_{c}+V_{h f}
$$

for some $V_{h f} \subset V$ which consists of $\delta$-algebraic high frequencies (see Definition 5.16. Namely, for any $v \in V$, there exists $v_{c} \in V_{c}$ and $v_{h f} \in V_{h f}$ such that

$$
v=v_{c}+v_{h f}, \quad\left\|v_{h f}\right\|_{A}^{2} \leq c_{1}\|v\|_{A}^{2} .
$$

Then the corresponding 2-level AMG satisfies

$$
\|E\|_{A} \leq 1-\frac{\delta}{c_{1}} .
$$




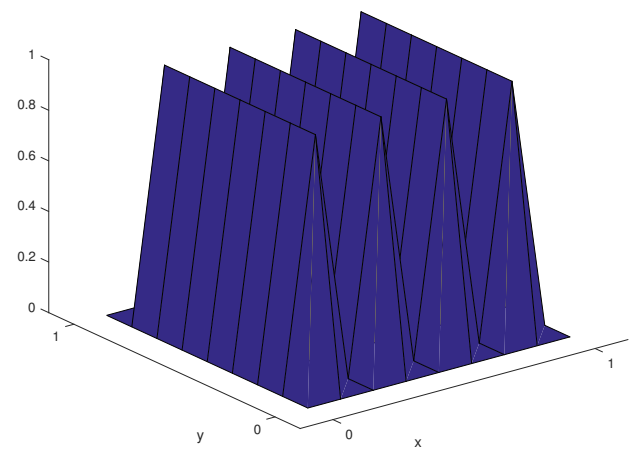

Figure 5.4. Graph of function defined in 5.50 . This function is highly oscillatory on the $x$ direction.

Proof. It follows that

$$
\inf _{w_{c} \in V_{c}}\left\|v-w_{c}\right\|_{\bar{R}^{-1}}^{2} \leq\left\|v_{h f}\right\|_{\bar{R}^{-1}}^{2} \leq \delta^{-1}\left\|v_{h f}\right\|_{A}^{2} \leq \frac{c_{1}}{\delta}\|v\|_{A}^{2} .
$$

As a result,

$$
K\left(V_{c}\right) \leq \frac{c_{1}}{\delta}
$$

and, finally, we have,

$$
\|E\|_{A}=1-\frac{1}{K\left(V_{c}\right)} \leq 1-\frac{\delta}{c_{1}}
$$

Corollary 5.20. If $V_{h f}$ consists of $\delta$-algebraic high frequencies, then for the coarse space $V_{c}$ given by

$$
V_{c}=\operatorname{Range}\left(I-P_{h f}\right),
$$

where $P_{h f}: V \mapsto V_{h f}$ is the A-orthogonal projection. Then

$$
\|E\|_{A} \leq 1-\delta .
$$

\subsection{Bibliographical notes}

One of the first results on two level convergence of AMG methods are found in earlier papers (Brandt et al. 1982b, Ruge and Stüben 1987). There have been a lot of research on reflecting the MG theory through algebraic settings: (Maitre and Musy 1983, Bank and Douglas 1985, Mandel 1988); algebraic variational approach to the two level MG theory (McCormick 1985, McCormick 1984, McCormick and Ruge 1982). 
For the two grid convergence, sharper results, including two sided bounds are given in (Zikatanov 2008) and also considered in (Falgout and Vassilevski 2004) and (Falgout, Vasilevski and Zikatanov 2005). These two-level results are more or less a direct consequences of the abstract theory provided in (Bramble et al. 1991c, $\mathrm{Xu}$ 1992, Xu and Zikatanov 2002). A survey of these and other related results is found in a recent article (MacLachlan and Olson 2014). The two approaches for analyzing that are included in this section were recently developed in $(\mathrm{Xu}$, Zhang and Zikatanov 2016c, Xu, Zhang and Zikatanov 2016a).

Theorem 5.2 can be found in (Zikatanov 2008) and can be viewed as a consequence of the XZ identity (Xu and Zikatanov 2002) in the special case of two subspaces from the general framework of the method of subspace corrections. The original proof of this theorem in (Zikatanov 2008) was based on the XZ identity. The proof here is new and is more direct.

Multilevel results are difficult to establish in general algebraic settings, and most of them are based on either not realistic assumptions or they use geometrical grids to prove convergence. We refer to (Vaněk, Mandel and Brezina 1996b, Brezina and Vassilevski 2011) for results in this direction. Rigorous multilevel results for finite element equations can be derived using the auxiliary space framework, which is developed in (Xu 1996) for quasi-uniform meshes. More recently multilevel convergence results for adaptively refined grids were shown to be optimal in (Chen, Nochetto and $\mathrm{Xu}$ 2012). A multilevel convergence result on shape regular grids using AMG based on quad-tree (in 2D) and oct-tree (in 3D) coarsening is shown in (Grasedyck, Wang and Xu 2015).

Finally, we point out that the notation used in parts of this section originates in (Bank and Dupont 1980, Bramble and Pasciak 1987, Bramble, Pasciak and Xu 1990) and is convenient for the analysis, especially when finite element equations are considered.

\section{A general approach to the construction of coarse space}

In this section, we describe an abstract framework for constructing coarse spaces by using the notion of space decomposition and subspace corrections.

Let us first introduce some technical results that are used as analytic tools later.

Lemma 6.1. Let $\underline{V}$ and $V$ be two vector spaces and let $\Pi: \underline{V} \mapsto V$ be a surjective map. Let $\underline{B}: \underline{V}^{\prime} \mapsto \underline{V}$ be an SPD operator. Then $B:=\Pi \underline{B} \Pi^{\prime}$ is also SPD. Furthermore

$$
\left(B^{-1} v, v\right)=\min _{\prod_{\mathcal{L}}=v}\left\langle\underline{B}^{-1} \mathcal{v} \mathcal{v}\right\rangle,
$$

with the unique minimizer given by

$$
\underline{\nu}^{*}=\underline{B} \Pi^{\prime} B^{-1} v .
$$

Lemma 6.2. Assume that following two conditions are satisfied for $\Pi$ : 
1. For all $\underline{v} \in \underline{V}$,

$$
\|\Pi \underline{v}\|_{A} \leq \tilde{\mu}_{1}\|\underline{v}\|_{\underline{B}^{-1}} .
$$

2. For any $v \in V$, there exists $\underline{v} \in \underline{V}$ such that $\Pi \underline{v}=v$ and

$$
\|v\|_{\underline{B}^{-1}} \leq \tilde{\mu}_{0}\|v\|_{A}
$$

Then

$$
\kappa(B A) \leq\left(\frac{\tilde{\mu}_{1}}{\tilde{\mu}_{0}}\right)^{2} .
$$

A direct consequence of the above Lemma 6.2 is the following result.

Theorem 6.3. (Fictitious Space Lemma) Assume that following two conditions are satisfied for П. First

$$
\|\Pi \underline{v}\|_{A} \leq \mu_{1}\|\underline{v}\|_{\underline{A}}, \quad \forall \underline{v} \in \underline{V}
$$

Secondly, for any $v \in V$, there exists $\underline{v} \in \underline{V}$ such that $\Pi \underline{v}=v$ and

$$
\|v\|_{\underline{A}} \leq \mu_{0}\|v\|_{A} .
$$

Then $\kappa(\Pi) \leq \mu_{1} / \mu_{0}$ and, under the assumptions of Lemma 6.1

$$
\kappa(B A) \leq\left(\frac{\mu_{1}}{\mu_{0}}\right)^{2} \kappa(\underline{B A}) .
$$

We assume there exist a sequence of spaces $V_{1}, V_{2}, \ldots, V_{J}$, which are not necessarily subspaces of $V$, but each of them is related to the original space $V$ by a linear operator

$$
\Pi_{j}: V_{j} \mapsto V
$$

We assume that $V$ can be written as a sum of subspaces and (4.12) and (4.13) hold.

Denote

$$
\underline{W}=V_{1} \times V_{2} \times \ldots \times V_{J},
$$

with the inner product

$$
(\underline{u}, \underline{v})=\sum_{i=1}^{J}\left(u_{i}, v_{i}\right),
$$

where $\underline{u}=\left(u_{1}, \ldots, u_{J}\right)^{T}$ and $\underline{v}=\left(v_{1}, \ldots, v_{J}\right)^{T}$. Or more generally, for $\underline{f}=\left(f_{1}, \ldots, f_{J}\right)^{T} \in$ $V^{\prime}$ with $f_{i} \in V_{i}^{\prime}$, we can define

$$
(\underline{f}, \underline{v})=\sum_{i=1}^{J}\left(f_{i}, v_{i}\right) .
$$


We now define $\Pi_{W}: \underline{W} \mapsto V$ by

$$
\Pi_{W} \underline{u}=\sum_{i=1}^{J} \Pi_{i} u_{i}, \quad \forall \underline{u}=\left(u_{1}, \ldots, u_{J}\right)^{T} \in \underline{W} .
$$

Formally, we can write

$$
\Pi_{W}=\left(\Pi_{1}, \ldots, \Pi_{J}\right) \text { and } \Pi_{W}^{\prime}=\left(\begin{array}{c}
\Pi_{1}^{\prime} \\
\vdots \\
\Pi_{J}^{\prime}
\end{array}\right) .
$$

We assume there is an operator $A_{j}: V_{j} \mapsto V_{j}^{\prime}$ which is symmetric, positive semi-definite for each $j$ and define $\underline{A}_{W}: \underline{W} \mapsto \underline{W}^{\prime}$ as follows

$$
\underline{A}_{W}:=\operatorname{diag}\left(A_{1}, A_{2}, \ldots, A_{J}\right) \text {. }
$$

For each $j$, we assume there is a symmetric positive definite operator $D_{j}: V_{j} \mapsto$ $V_{j}^{\prime}$, and define $\underline{D}: \underline{W} \mapsto \underline{W}^{\prime}$ as follows

$$
\underline{D}:=\operatorname{diag}\left(D_{1}, D_{2}, \ldots, D_{J}\right) \text {. }
$$

We associate a coarse space $V_{j}^{c}, V_{j}^{c} \subset V_{j}$, with each of the spaces $V_{j}$, and consider the corresponding orthogonal projection $Q_{j}: V_{j} \mapsto V_{j}^{c}$ with respect to $(\cdot, \cdot)_{D_{j}}$. We define $\underline{Q}: \underline{W} \mapsto \underline{W}^{\prime}$ by

$$
\underline{Q}:=\operatorname{diag}\left(Q_{1}, Q_{2}, \ldots, Q_{J}\right)
$$

\section{Assumption 6.9.}

1 The following inequality holds for all $\underline{w} \in \underline{W}$ :

$$
\left\|\Pi_{W} \underline{w}\right\|_{D}^{2} \leq C_{p, 2}\|\underline{w}\|_{D}^{2},
$$

for some positive constant $C_{p, 2}$.

2 For each $w \in V$, there exists a $\underline{w} \in \underline{W}$ such that $w=\Pi_{W} \underline{w}$ and the following inequality holds

$$
\|\underline{w}\|_{\underline{A}_{W}}^{2} \leq C_{p, 1}\|w\|_{A}^{2}
$$

with a positive constant $C_{p, 1}$ independent of $w$.

3 For all $j$,

$$
N\left(A_{j}\right) \subset V_{j}^{c} .
$$

Remark 6.4. The above assumption implies that

$$
w \in N(A) \Rightarrow \underline{w} \in N\left(A_{1}\right) \times \ldots \times N\left(A_{J}\right) .
$$

We define the global coarse space $V_{c}$ by

$$
V_{c}:=\sum_{j=1}^{J} \Pi_{j} V_{j}^{c} .
$$


Further, for each coarse space $V_{j}^{c}$, we define

$$
\mu_{j}^{-1}\left(V_{j}^{c}\right):=\max _{v_{j} \in V_{j}} \min _{v_{j}^{c} \in V_{j}^{c}} \frac{\left\|v_{j}-v_{j}^{c}\right\|_{D_{j}}^{2}}{\left\|v_{j}\right\|_{A_{j}}^{2}},
$$

and

$$
\mu_{c}=\min _{1 \leq j \leq J} \mu_{j}\left(V_{j}^{c}\right),
$$

which is finite, thanks to Assumption 6.9.3 (namely, 6.12).

By the two level convergence theory, if $D_{j}$ provides a convergent smoother, then $\left(1-\mu_{j}\left(V_{j}^{c}\right)\right)$ is the convergence rate for two-level AMG method for $V_{j}$ with coarse space $V_{j}^{c}$. Next theorem gives an estimate on the convergence of the two level method in terms of the constants from Assumptions 6.9 and $\mu_{c}$.

Theorem 6.5. If Assumption 6.9 holds, then for each $v \in V$, we have the following error estimate

$$
\min _{v_{c} \in V_{c}}\left\|v-v_{c}\right\|_{D}^{2} \leq C_{p, 1} C_{p, 2} \mu_{c}^{-1}\|v\|_{A}^{2} .
$$

Proof. By Assumption 6.9, for each $v \in V$, there exists $\underline{v} \in \underline{V}$ such that

$$
v=\Pi_{W} \underline{v}
$$

and 6.11$)$ is satisfied.

By the definition of $\mu_{c}$, we have

$$
\|\underline{v}-\underline{Q} \underline{v}\|_{\underline{D}}^{2} \leq \mu_{c}^{-1}\|\underline{v}\|_{\underline{A}_{W}}^{2} .
$$

We let $v_{c}=\Pi_{W} \underline{\underline{v}}$. Then $v_{c} \in V_{c}$ and by Assumption 6.9, we have

$\left\|v-v_{c}\right\|_{D}^{2}=\| \Pi_{W}\left(\underline{v}-\underline{Q} \underline{v}\left\|_{D}^{2} \leq C_{p, 2}\right\| \underline{v}-\underline{Q} \underline{v}\left\|_{\underline{D}}^{2} \leq C_{p, 2} \mu_{c}^{-1}\right\| \underline{v}\left\|_{A_{W}}^{2} \leq C_{p, 1} C_{p, 2} \mu_{c}^{-1}\right\| v \|_{A}^{2}\right.$.

$\square$

We define another product space

$$
\underline{V}:=V_{c} \times V_{1} \times V_{2} \times \cdots \times V_{J},
$$

and we set $\Pi_{c}: V_{c} \mapsto V$ to be the natural inclusion from $V_{c}$ to $V$. Then we define $\Pi: \underline{V} \mapsto V$ by

$$
\Pi:=\left(\Pi_{c} \Pi_{1} \Pi_{2} \cdots \Pi_{J}\right)
$$

and $\underline{A}: \underline{V} \mapsto \underline{V}^{\prime}$ by

$$
\underline{A}_{:}=\left(\begin{array}{llll}
A_{c} & & & \\
& A_{1} & & \\
& & \ddots & \\
& & & A_{J}
\end{array}\right) \text {, }
$$


where $A_{c}: V_{c} \mapsto V_{c}^{\prime}$ is given as

$$
A_{c}:=\Pi_{c}^{\prime} A P i_{c} .
$$

And $\underline{B}: \underline{V} \mapsto \underline{V}^{\prime}$ is given as

$$
\underline{B}:=\left(\begin{array}{ccccc}
A_{c}^{-1} & & & & \\
& D_{1}^{-1} & & & \\
& & D_{2}^{-1} & & \\
& & & \ddots & \\
& & & & D_{J}^{-1}
\end{array}\right),
$$

We introduce the additive preconditioner $\widehat{B}$

$$
\widehat{B}:=\Pi \underline{B} \Pi^{\prime}=\Pi_{c} A_{c}^{-1} \Pi_{c}^{\prime}+\sum_{j=1}^{J} \Pi_{j} D_{j}^{-1} \Pi_{j}^{\prime},
$$

and we have the following results.

Lemma 6.6. If Assumption 6.9 holds, then for any $v \in V$, there exists $\underline{v} \in \underline{V}$ such that 6.4 holds, namely

$$
\|v\|_{\underline{B}^{-1}} \leq \tilde{\mu}_{0}\|v\|_{A}
$$

with $\tilde{\mu}_{0}$ being a constant depending on $C_{p, 1}, C_{p, 2}, \mu_{c}$ and $c^{D}$.

Lemma 6.7. If Assumption 6.10 holds, then 6.3 holds with constant $\tilde{\mu}_{1}$ depends on $C_{p, 2}$ and $c^{D}$.

By directly applying Lemma 6.2, we immediately have

Theorem 6.8. If Assumption 6.9 holds, then

$$
\kappa(\widehat{B} A) \leq\left(\frac{\tilde{\mu}_{1}}{\tilde{\mu}_{0}}\right)^{2} .
$$

The following two-level convergence result is an application of the convergence theorem (Theorem 5.2) with the error estimate in Theorem 6.5.

Theorem 6.9. If Assumption 6.9 holds. Then the two-level AMG method with coarse space defined in 6.13) converges with a rate

$$
\|E\|_{A}^{2} \leq 1-\frac{\mu_{c}}{C_{p, 1} C_{p, 2} c^{D}}
$$

\subsection{Bibliographical notes}

The fictitious space Lemma was first proved in (Matsokin and Nepomnyashchikh 1985). Related is also the work on auxiliary space method (Xu 1996). Additive version of Lemma 6.1 is found in (Xu and Zikatanov 2002), and the most general case (including multiplicative preconditioners) is in (Xu 2016). 
In later sections we show how the general theory used here can be applied to various AMG algorithms, e.g. classical AMG (Brandt et al. 1982b, Ruge and Stüben 1987), smoothed aggregation AMG (Míka and Vaněk 1992b, Míka and Vaněk 1992a), spectral AMGe (Chartier, Falgout, Henson, Jones, Manteuffel, McCormick, Ruge and Vassilevski 2003, Efendiev, Galvis and Vassilevski 2011), and other algorithms.

Many of the works in the Domain Decomposition (DD) literature also use techniques for defining coarse spaces, which, to a large extend, have similar aims as the coarse space constructions for AMG outlined in this section. We refer to (Toselli and Widlund 2005, Widlund 2009, Widlund 1994, Dohrmann, Klawonn and Widlund 2008, Spillane, Dolean, Hauret, Nataf, Pechstein and Scheichl 2014) and the references therein for more details on using local eigenspaces for constructing coarse space in DD methods.

\section{Graphs and sparse matrices}

In this section, we give a brief introduction to some basic notion of graph theory that is often used for sparse matrices and also for the study of AMG.

\subsection{Sparse matrix and its adjacency graph}

An undirected graph (or simply a graph) $\mathcal{G}$ is a pair $(\mathcal{V}, \mathcal{E}$ ), where $\mathcal{V}$ is a finite set of points called vertices and $\mathcal{E}$ is a finite set of pairs of vertices, known as edges. We often write $\mathcal{V}=\{1, \ldots, n\}$ for some fixed $n$. We will not consider directed graphs in this article because the graphs corresponding to the symmetric sparse matrices are undirected.

An edge $e \in \mathcal{E}$ is an unordered pair $(j, k)$, where $j, k \in \mathcal{V}$. The vertices $j$ and $k$ are said to be adjacent if $(j, k) \in \mathcal{E}$. A path from a vertex $j$ to a vertex $k$ is a sequence $\left(j_{0}, j_{1}, j_{2}, \ldots, j_{l}\right)$ of vertices where $j_{0}=j, j_{l}=k$, and $\left(j_{i}, j_{i+1}\right) \in \mathcal{E}$ for all $i=0,1, \ldots, l-1$. A vertex $j$ is connected to a vertex $k$ if there is a path from $j$ to $k . \mathcal{G}=(\mathcal{V}, \mathcal{E})$ is connected if every pair of vertices is connected by a path, otherwise it is said to be disconnected. A graph $\mathcal{G}_{0}=\left(\mathcal{V}_{0}, \mathcal{E}_{0}\right)$ is called a subgraph of $\mathcal{G}=(\mathcal{V}, \mathcal{E})$ if $\mathcal{V}_{0} \subset \mathcal{V}$ and $\mathcal{E}_{0} \subset \mathcal{E}$.

The neighborhood $N(i)$ are the vertices adjacent to the vertex $i$. The degree or valency of a vertex is the number of edges that connect to it. These are defined as:

$$
N(i)=\{j:(i, j) \in \mathcal{E}\}, \quad d_{i}=|\{j:(i, j) \in \mathcal{E}\}| .
$$

A path connecting two vertices $i$ and $j$ is a sequence of edges $\left(k_{0}, k_{1}\right),\left(k_{1}, k_{2}\right), \ldots$, $\left(k_{m-1}, k_{m}\right)$ in $\mathcal{E}$ such that $k_{0}=i$ and $k_{m}=j$. The length of the path is the number of edges in it. The distance between two vertices $i$ and $j$ is the length of the shortest path connecting $i$ and $j$, and we denote it by $\operatorname{dist}(i, j)$. If $i, j$ are not connected, then $\operatorname{dist}(i, j)=\infty$. The diameter of a graph is the largest distance between two vertices, i.e. $\operatorname{diam}(\mathcal{G})=\max _{(i, j) \in \mathcal{E}} \operatorname{dist}(i, j)$. An independent set is a set of vertices in which no 
two of which are adjacent. A maximal independent set is an independent set such that adding any other vertex to the set forces the set to contain an edge.

Given a symmetric matrix $A \in \mathbb{R}^{n \times n}$, the adjacency graph of $A$ is an undirected graph, denoted by $\mathcal{G}(A), \mathcal{G}=(\mathcal{V}, \mathcal{E})$ with $\mathcal{V}=\{1,2, \ldots, n\}$. The edges $\mathcal{E}$ are defined as

$$
\mathcal{E}=\left\{(j, k) \mid a_{j k} \neq 0\right\}
$$

A matrix $A$ is called irreducible if its adjacency graph $\mathcal{G}(A)=(\mathcal{V}, \mathcal{E})$ is connected. Otherwise, $A$ is called reducible.

An example of a symmetric matrix is shown in Figure 7.5 (left) and a drawing of the corresponding graph is in Figure 7.5 (right). The pictorial representation of a graph is often not available and a graph can be drawn in different ways with different coordinates of the vertices. As a general rule, sparse matrices do not provide any geometrical information for the underlying graph and only the combinatorial/topological properties of $\mathcal{G}(A)$.

$$
A=\left(\begin{array}{llllll}
* & * & * & * & * & * \\
* & * & * & 0 & 0 & 0 \\
* & * & * & * & * & 0 \\
* & 0 & * & * & * & 0 \\
* & 0 & * & * & * & 0 \\
* & 0 & 0 & 0 & 0 & *
\end{array}\right)
$$

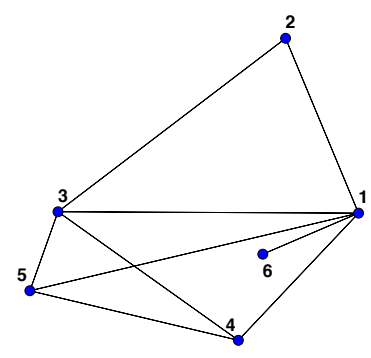

Figure 7.5. A sparse symmetric matrix (left) and its associated graph (right).

Given

$$
S \subset\{1, \ldots, n\} \times\{1, \ldots, m\}
$$

we define

$$
\mathbb{R}_{S}^{n \times m}=\left\{X=\left(x_{i j}\right) \in \mathbb{R}^{n \times m}: x_{i j}=0 \text { if }(i, j) \notin S\right\}
$$

We say that $X$ has sparsity pattern given by $S$ if and only if $X \in \mathbb{R}_{S}^{n \times m}$.

Often, the sparsity pattern of a matrix is determined in advance and the set $S$ is determined by a given matrix. For $Y \in \mathbb{R}^{n \times m}$ we denote

$$
\mathrm{S}(Y)=\left\{(i, j) \mid y_{i j} \neq 0\right\} .
$$

We now consider the graphs associated with finite element or finite difference stiffness matrices. In the case of finite elements, this is the space of FE functions 
$V_{h}$, and, for finite difference discretizations this is the space of mesh-functions $V_{h}$, which can be identified with $\mathbb{R}^{N}$.

We assume that we have a finite dimensional space $V_{h}^{N}$ which we use to discretize the Neumann problem. We also have the FE space for the Dirichlet (or mixed boundary conditions) problem and we assume that the following inclusions hold $V_{h}=V_{h}^{D} \subset V_{h}^{N}$. Equivalently, we have that a subspace of the degrees of freedom vanishes on $V_{h}$ : for example, the values of the finite element or finite difference solution at the nodes on the Dirichlet boundary vanish.

Let $A^{\mathcal{N}}$ be the matrix corresponding to the finite element or finite difference discretization of the model second order elliptic equation with Neumann boundary conditions. Clearly we have the following identity,

$$
\left(A^{\mathcal{N}} u, v\right)=\sum_{e \in \mathcal{E}} \omega_{e} \delta_{e} u \delta_{e} v
$$

Here, the sum is over all edges $\mathcal{E}$ of the graph $\mathcal{G}\left(A^{\mathcal{N}}\right)=(\mathcal{V}, \mathcal{E}), \delta_{e} v=v_{i}-v_{j}$ if $\mathcal{E} \ni e=(i, j), i<j$. Also, $\omega_{e}=-\left(A^{\mathcal{N}}\right)_{i j}$ are the off-diagonal entries of $A^{\mathcal{N}}$. Note that, since we consider the Neumann problem, the bilinear form defined by $A^{\mathcal{N}}$ vanishes for $u$ (resp. v) such that $u_{i}=1$ (resp. $v_{i}=1$ ) for all $i$. For both 5-point and 9-point stencils we have that $\omega_{e}=1$ for all $e$.

We consider the stiffness matrix $A^{\mathcal{N}}$, corresponding to the model problem 2.1) with Neumann boundary conditions on a bounded domain $\Omega \subset \mathbb{R}^{d}$, namely we have boundary condition:

$$
\alpha \nabla u \cdot \boldsymbol{n}=0, \quad \text { on } \quad \partial \Omega,
$$

where $\boldsymbol{n}$ is the unit normal vector to $\partial \Omega$ pointing outward. It is easy to derive the stiffness matrices corresponding to the Dirichlet or mixed boundary condition problem: we just restrict the bilinear form defined by $A^{\mathcal{N}}$ to a subspace:

$$
(A u, v)=\sum_{e \in \mathcal{E}} \omega_{e} \delta_{e} u \delta_{e} v, \quad u_{j}=v_{j}=0 \quad x_{j} \in \Gamma_{D} .
$$

Remark 7.1. Similar relations between differential problems with natural (Neumann) and essential (Dirichlet) boundary conditions are seen not only for the model problem considered here, but also for problems on $H(\mathrm{curl}), H($ div $)$, linear elasticity and other.

\subsection{M-matrix relatives of finite element stiffness matrices}

A symmetric matrix $A \in \mathbb{R}^{n \times n}$ is called an $M$-matrix if it satisfies the following three properties:

$$
\begin{aligned}
& a_{i i}>0 \text { for } i=1, \ldots, n, \\
& a_{i j} \leq 0 \text { for } i \neq j, \quad i, j=1, \ldots, n, \\
& A \text { is semi-definite. }
\end{aligned}
$$


As first step in creating space hierarchy the majority of the AMG algorithms for $A u=f$ with positive semidefinite $A$ uses a simple filtering of the entries of $A$ and construct an $M$-matrix which is then used to define crucial AMG components.

Definition 7.2. ( $M$-matrix relative) We call a matrix $\widetilde{A}$ an $M$-matrix relative of $A$ if $\widetilde{A}$ is an M-matrix and satisfies the inequalities

$$
(v, v)_{\widetilde{A}} \lesssim(v, v)_{A}, \quad \text { and } \quad(v, v)_{D} \lesssim(v, v)_{\widetilde{D}}, \quad \text { for all } \quad v \in V,
$$

where $\widetilde{D}$ and $D$ are the diagonals of $\widetilde{A}$ and $A$ respectively.

A few remarks are in order: (1) We have used the term $M$-matrix to denote semidefinite matrices, and we are aware that this is not the precise definition. It is however much more convenient to use reference to $M$-matrices and we decided to relax a bit the definition here with the hope that such inaccuracy pays off by better appeal to the reader; (2) We point out that the restricted $M$-matrix relatives are instrumental in the definition of coarse spaces and also in the convergence rate estimates. This is clearly seen later in $\$ 5.2$ where we present the unified two level theory for AMG. (3) Often, the case is that the one sided inequality in (7.9) is in fact a spectral equivalence.

By definition, we have the following simple but important result.

Lemma 7.3. Let $A_{+}$be an $M$-matrix relative of $A$ and let $D$ and $D_{+}$be the diagonal matrices of $A$ and $A_{+}$, respectively. If $V_{c} \subset V$ is a subspace, then the estimate

$$
\left\|u-u_{c}\right\|_{D}^{2} \lesssim\|u\|_{A}^{2}
$$

holds for some $u_{c} \in V_{c}$, if the estimate

$$
\left\|u-u_{c}\right\|_{D_{+}}^{2} \lesssim\|u\|_{A_{+}}^{2}
$$

holds.

This result means that we only need to work on the M-matrix relative of $A$ in order to get the estimate (7.10).

In this section we show how to construct $M$-matrix relative to the matrix resulting from a finite element discretization of the model problem (2.1) with linear elements. We consider first an isotropic problem with Neumann boundary condition (7.4) and isotropic $\alpha=a(x) I$. Construction of M-matrix relatives in the case of anisotropic tensor $\alpha(x)$ in $(2.1)$ is postponed to $\$ 14.2$.

In the rest of this section, we make the following assumptions on the coefficient and the geometry of $\Omega$ :

- The domain $\Omega \subset \mathbb{R}^{d}$ is partitioned into simplices $\Omega=\cup_{T \in \mathcal{T}_{h}} T$.

- The coefficient $a(x)$ is a scalar valued function and its discontinuities are aligned with the partition $\mathcal{T}_{h}$. 
- We consider the Neumann problem, and, therefore, the bilinear form (2.3) is

$$
\int_{\Omega} a(x) \nabla v \cdot \nabla u=\sum_{(i, j) \in \mathcal{E}}\left(-a_{i j}\right) \delta_{e} u \delta_{e} v=\sum_{e \in \mathcal{E}} \omega_{e} \delta_{e} u \delta_{e} v .
$$

- It is well known that the off-diagonal entries of the stiffness matrix $A$ are given by

$$
\begin{aligned}
& \omega_{e}=-\left(\phi_{j}, \phi_{i}\right)_{A}=\sum_{T \supset e} \omega_{e, T} \\
& \omega_{e, T}=\frac{1}{d(d-1)} \bar{a}_{T}\left|\kappa_{e, T}\right| \cot \alpha_{e, T}, \quad \bar{a}_{T}=\frac{1}{|T|} \int_{T} a(x) d x .
\end{aligned}
$$

Here, $e=(i, j)$ is a fixed edge with end points $x_{i}$ and $x_{j} ; T \supset e$ is the set of all elements containing $e ;\left|\kappa_{e, T}\right|$ is the volume of $(d-2)$-dimensional simplex opposite to $e$ in $T ; \alpha_{e, T}$ is the dihedral angle between the two faces in $T$ not containing $e$.

- Let $\mathcal{E}$ denote the set of edges in the graph defined by the triangulation and let $\mathcal{E}^{-}$be the set of edges where $a_{i j} \geq 0, i \neq j$. The set complementary to $\mathcal{E}^{-}$is $\mathcal{E}^{+}=\mathcal{E} \backslash \mathcal{E}^{-}$. Then, with $\omega_{e}=-a_{i j}$, and, $\delta_{e} u=\left(u_{i}-u_{j}\right), e=(i, j)$ we have

$$
\int_{\Omega} a(x) \nabla v \cdot \nabla u=\sum_{e \in \mathcal{E}^{+}} \omega_{e} \delta_{e} u \delta_{e} v-\sum_{e \in \mathcal{E}^{-}}\left|\omega_{e}\right| \delta_{e} u \delta_{e} v .
$$

- We also assume that the partitioning is such that the constant function is the only function in the null space of the bilinear form (7.12). This is, of course, the case when $\Omega$ is connected (which is true, as $\Omega$ is a domain).

The non-zero off-diagonal entries of $A$ may have either positive or negative sign, and, usually $\mathcal{E}^{-} \neq \emptyset$. The next theorem shows that the stiffness matrix $A$ defined via the bilinear form 7.12 is spectrally equivalent to the matrix $A_{+}$defined as

$$
\left(A_{+} u, v\right)=\sum_{e \in \mathcal{E}^{+}} \omega_{e}\left(u_{i}-u_{j}\right)\left(v_{i}-v_{j}\right)
$$

Thus, we can ignore any positive off-diagonal entries in $A$, or equivalently, we may drop all $\omega_{e}$ for $e \in \mathcal{E}^{-}$. Indeed, $A_{+}$is obtained from $A$ by adding to the diagonal all positive off diagonal elements and setting the corresponding off-diagonal elements to zero. This is a stronger result that we need later, because it gives spectral equivalence with the $M$-matrix relative $A_{+}$.

Theorem 7.4. If $A$ is the stiffness matrix corresponding to linear finite element discretization of (2.1) with boundary conditions given by (7.4). Then $A_{+}$is an $M$-matrix relative of $A$ which is spectrally equivalent to $A$. The constants of equivalence depend only on the shape regularity of the mesh. Moreover, the graph corresponding to $A_{+}$is connected.

A simple corollary which we use later in proving estimates on the convergence rate is as follows. 
Corollary 7.5. Assume that $A$ is the stiffness matrix for piece-wise linear discretization of equation (7.12) and $A_{+}$is the $M$-matrix relative defined in Theorem 7.4 Then the diagonal $D$ of $A$ and the diagonal $D_{+}$of $A_{+}$are spectrally equivalent.

Proof. For the diagonal elements of $A$ and $A_{+}$we have

$$
[D]_{j}=\left(\phi_{j}, \phi_{j}\right)_{A} \equiv\left(\phi_{j}, \phi_{j}\right)_{A_{+}}=\left[D_{+}\right]_{j} .
$$

The equivalences written above follow directly from Lemma 7.4 . $\square$

Corollary 7.5 together with Lemma 7.3 provide a theoretical foundation for using M-matrix relative to design AMG for finite element matrices.

\subsection{Bibliographical notes}

We have introduced some standard notions from graph theory. For the reader interested in more details descriptions, we refer to classical textbooks (Diestel 2010, Gibbons 1985) as general introduction on graph theory; and to (Saad 2003a), (Varga 2000) for considerations linking graphs, sparse matrices and iterative methods.

Our results on $M$-matrix relatives are related to the some of the works on preconditioning by $Z$-matrices and $L$-matrices (Kraus and Schicho 2006, Kraus 2008). They are implicitly used in most of the AMG literature (Ruge and Stüben 1987) where the classical strength of connection definition gives an $M$-matrix. We point out that the $M$-matrix property and the existence of $M$-matrix relative is often not sufficient to achieve even a two-level uniform convergence of AMG. A typical example is a matrix which has been re-scaled and the constant is not in the kernel of the discrete operator anymore. In such case, the standard AMG application may fail, and the near kernel needs to be recovered by different means, such as the adaptive AMG processes considered in $\$ 15$ and the references given there-in.

\section{Strength of connections}

A central task in AMG is to obtain an appropriate coarse space or prolongation. This process is known as a coarsening process. In a geometric grid or, more generally, the adjacency graph of the stiffness matrix, we need to identify vertices to be deleted from the graph. We need the coarsened graph still provide a good approximation for algebraically low frequencies.

\subsection{Basic idea and strength function}

If a subset of vertices on which an algebraically smooth vector, say $v$, change very slowly, we only need to keep one degree of freedom to represent $v$ in this subset. In other words, we can either aggregate this subset together or keep one of vertex and delete the rest of vertices in this subset. We say vertices in this subset are strongly connected to each other. The strength of connection is a concept introduced to 
identify strongly connected pairs of vertices. Roughly speaking, we say $i$ and $j$ is strongly connected if $v_{i} \approx v_{j}$.

We imagine to coarsen the graph with two different steps: first step is to remove some edges and the second step is to remove some vertices. The second step is the goal. Let us exam how the second step is carried out: we either (1) aggregate some neighboring vertices together or (2) pick an MIS, denoted as $C$, in the filtered graph and then remove all the remaining vertices. Using the argument above, (1) each aggregate should only consists of strongly connected, or (2) each one of all the deleted vertices should be strongly connected to some point in $C$. To guarantee either of these two situations, we then have to remove all the weakly connected edges in the first step.

Let us further use some heuristic arguments to motivate how the strength of connection should be defined. Let $v$ be an algebraically smooth (5.41), namely

$$
\|v\|_{A}^{2} \leq \epsilon\|v\|_{\bar{R}^{-1}}^{2} .
$$

Let $u=v /\|v\|_{\bar{R}^{-1}}$, we then have

$$
(A u, u) \leq \epsilon \Rightarrow \sum_{e=(i, j) \in \mathcal{E}}\left(-a_{i j}\right)\left(u_{i}-u_{j}\right)^{2} \leq \epsilon .
$$

Thanks to Lemma 5.15, we can assume that $A$ is an M-matrix, namely $-a_{i j}=\left|a_{i j}\right|$. It follows from (8.1), we have the following observations:

1 A larger $\left|a_{i j}\right|$ means a smaller $\left(u_{i}-u_{j}\right)^{2}$;

2 An algebraically smooth error varies more slowly in the direction where $\left|a_{i j}\right|$ is larger;

The observation leads to the following definition of the strength of connection: Given a threshold $\theta>0$, we say that the vertex $j$ of the adjacency graph is $\theta$ strongly connected to vertex $i$ if

$$
-a_{i j} \geq \theta \max _{k \neq i}-a_{i k}
$$

We note that, by the definition above we may have $j$ strongly connected to $i$, while $i$ is not strongly connected to $j$. As a result, the adjacency graph corresponding to the matrix with the strong connections may not be symmetric.

But our theoretical framework is given in terms of the symmetrized operator $\bar{R}$, regardless if the original smoother $R$ is symmetric or not. This is due to the fact that we used the energy norm, namely $A$-norm, to measure the convergence rate and the resulting convergence rate is given in terms of $\bar{R}$. This is the best convergence theory we have, and we will use this theory to study AMG algorithms. As a result, we will only consider the strength functions that are symmetric. Associated with an SSPD matrix, a strength function is such that

$$
s_{c}: \mathcal{V} \times \mathcal{V} \mapsto \mathbb{R}_{+},
$$


which is symmetric, namely $s_{c}(i, j)=s_{c}(j, i)$.

Given a threshold $\theta>0$, we say $i$ and $j$ are $\theta$-strongly connected if

$$
s_{c}(i, j) \geq \theta \text {. }
$$

We then define the strength matrix:

$$
S=\sum_{s_{c}(i, j) \geq \theta} e_{i} e_{j}^{T}
$$

Notice that $S$ is a boolean matrix with entries equal to 0 or 1 depending on the strength of connection.

Consider non-overlapping decomposition

$$
\mathcal{V}=\bigcup_{i=1}^{m} \mathcal{A}_{i}=\bigcup_{\tilde{\mathcal{A}} \in \mathcal{V}_{\mathcal{H}}} \tilde{\mathcal{A}}, \quad \mathcal{V}_{\mathcal{A}}=\left(\mathcal{A}_{1}, \ldots, \mathcal{A}_{m}\right) .
$$

We extend the definition of strength function

$$
s_{c}: \mathcal{V}_{\mathcal{A}} \times \mathcal{V}_{\mathcal{A}} \mapsto \mathbb{R}_{+} .
$$

and we assume that $s_{c}$ is symmetric.

Given a threshold $\theta>0$, we say $\mathcal{A}_{i}$ and $\mathcal{A}_{j}$ are $\theta$-strongly connected to each other if

$$
s_{c}\left(\mathcal{A}_{i}, \mathcal{A}_{j}\right) \geq \theta .
$$

An example of such a strength function is given in (8.13).

In the AMG literature, a number of heuristics have been proposed for identifying strong connections, particularly when considering discretizations of anisotropic equations. In general, the strength of connection is a notion that is difficult to address theoretically or to relate it with the convergence rate of an algorithm. We refer to the classical papers and monographs mentioned in $\$ 9.6$ for further discussions on related issues. Current trends in AMG development aim to re-evaluate the role of the classical definition of the strength of connection.

We would finally like to comment that the strength of connection is used to define the sparsity of $P$ and it is crucial, for example, in the proof of the convergence of the two-level method for discretizations of elliptic equation with jump coefficients. Choosing the "right" sparsity of $P$ is crucial as a denser $P$ would lead to a better approximation from a coarser space; and a sparser $P$ would lead to a less expensive algorithm.

In the rest of section, we discuss different definitions of strength of connections:

1 classical AMG;

2 lean $\mathrm{AMG}$

3 local-optimization based. 


\subsection{Classical AMG}

With the above motivation, we define the strength function as follows:

$$
\begin{aligned}
s_{c}(i, j) & =\frac{-a_{i j}}{\min \left(\max _{k \neq i}\left(-a_{i k}\right), \max _{k \neq j}\left(-a_{j k}\right)\right)} \\
& =\frac{a_{i j}}{\max \left(\min _{k \neq i} a_{i k}, \min _{k \neq j} a_{j k}\right)} .
\end{aligned}
$$

The definition (8.7) is symmetrized version of strength function used in the classical AMG literature (see (8.2)).

The following definition is also commonly used in classical AMG algorithms

$$
s_{c}(i, j)=\frac{\left|a_{i j}\right|}{\min \left(\frac{1}{|N(i)|} \sum_{k \neq i}\left|a_{i k}\right|, \frac{1}{|N(j)|} \sum_{k \neq i}\left|a_{j k}\right|\right)} .
$$

Again, this is a symmetrized version of strength functions used in the AMG literature.

Finally we may also have the following two definitions which are based on Cauchy-Schwarz for SSPD matrices:

$$
s_{1}(i, j)=\frac{\left|a_{i j}\right|}{\sqrt{a_{i i} a_{j j}}}
$$

and

$$
s_{2}(i, j)=\frac{-2 a_{i j}}{a_{i i}+a_{j j}}
$$

Note that the definition (8.7) (which is mostly associated with Classical AMG) ignores all the non-negative entries of the stiffness matrix $A=\left(a_{i j}\right)$.

\subsection{Local-optimization strength function}

Suppose that each pair of the index $\{i, j\} \subset\{1, \ldots, n\}$ is associated with a space $V_{i j}$, which is not necessarily a subspace of $V$. Assume now we have two operators: $A_{i j}: V_{i j} \mapsto V_{i j}^{\prime}$ which is symmetric positive, semi-definite and $D_{i j}: V_{i j} \mapsto V_{i j}^{\prime}$ which is SPD.

Given a number $k_{i j}<\operatorname{dim} V_{i j}$, we define a coarse space $V_{i j}^{c} \subset V_{i j}$ as follows

$$
V_{i j}^{c}:=\operatorname{span}\left\{\zeta_{i j}^{(k)}, k=1: k_{i j}\right\}
$$

where $\zeta_{i j}^{(k)}$ is the eigenvector corresponds to the $k$-th smallest eigenvalue of $D_{i j}^{-1} A_{i j}$. We denote $Q_{i j}: V_{i j} \mapsto V_{i j}^{c}$ to be the orthogonal projection with respect to $(\cdot, \cdot)_{D_{i j}}$. 
Motivated by (6.14), we define the strength function $s_{c}$ as follows

$$
s_{c}(i, j):=\left(\sup _{v \in V_{i j}} \frac{\left\|\left(I-Q_{i j}\right) v\right\|_{D_{i j}}^{2}}{\|v\|_{A_{i j}}^{2}}\right)^{-1} .
$$

A special case of the above definition is introduced in AGMG. Suppose now we have a set of aggregates $\left\{\mathcal{A}_{1}, \ldots, \mathcal{A}_{J}\right\}$. We fix a pair $\{i, j\} \subset\{1, \ldots, J\}$, and define

$$
G=\mathcal{A}_{i} \bigcup \mathcal{A}_{j}
$$

We then define by $V_{i j}$ the restriction of $V$ on $G$ as follows

$$
V_{i j}:=\left\{\left.v\right|_{G}: v \in V\right\},
$$

where

$$
\left.v\right|_{G}(x)= \begin{cases}v(x), & \text { if } x \in G, \\ 0, & \text { if } x \notin G .\end{cases}
$$

We denote the restriction of $A$ and $D$ on $G$ by $A_{i j}$ and $D_{i j}$ respectively. We then choose $k_{i j}=1$ and define the local coarse space $V_{i j}^{c}$

$$
V_{i j}^{C}=\operatorname{span}\left\{\zeta_{G}\right\}, \quad \zeta_{G}=\zeta_{i j}^{(1)} .
$$

and the orthogonal projection $Q_{i j}: V_{i j} \mapsto V_{i j}^{C}$ with respect to $(\cdot, \cdot)_{D_{i j}}$, namely,

$$
Q_{i j} v=\frac{\left(v, \zeta_{G}\right)_{D_{i j}}}{\left\|\zeta_{G}\right\|_{D_{i j}}^{2}} \zeta_{G}
$$

The strength function based on aggregation is defined as follows:

$$
s_{c}(i, j):=\left(\sup _{v \in V_{i j}} \frac{\left\|\left(I-Q_{i j}\right) v\right\|_{D_{i j}}^{2}}{\|v\|_{A_{i j}}^{2}}\right)^{-1} .
$$

Another example is choosing $V_{i j}=\mathbb{R}^{2}$ and

$$
A_{i j}=\left(\begin{array}{cc}
a_{i i} & a_{i j} \\
a_{i j} & a_{j j}
\end{array}\right), \quad D_{i j}=\left(\begin{array}{cc}
a_{i i} & 0 \\
0 & a_{j j}
\end{array}\right) .
$$

We choose $k_{i j}=1$ and define the coarse space $V_{i j}^{c} \subset V_{i j}$ as follows

$$
V_{i j}^{c}=\operatorname{span}\left\{\left(\begin{array}{l}
1 \\
1
\end{array}\right)\right\} \text {. }
$$

By a direct computation, we have the strength function defined in 8.11 is

$$
s_{c}(i, j)=\frac{1-s_{1}^{2}}{1-s_{2}}, \quad s_{1}=\frac{\left|a_{i j}\right|}{\sqrt{a_{i i} a_{j j}}}, \quad s_{2}=-\frac{2 a_{i j}}{a_{i i}+a_{j j}} .
$$


We notice that $s_{1} \geq s_{2}$ and hence

$$
s_{c}(i, j) \leq 1+s_{2} \leq 1+s_{1} .
$$

We would like to point out that the strength function given by $(8.14)$ is obtained by using the theory in $\$ 6$, while the other strength functions such as 8.7 are obtained by heuristic considerations.

\subsection{Lean $A M G$}

Instead of using the absolute value of matrix entries as the criteria to determine if two points are strongly coupled, Lean AMG use the affinity to measure the strength of connections, which is based on the following heuristic observation:

Given a vector $v$, if $(i, j)$ is a strong connected pair of vertices, after several relaxation on $v$, namely

$$
v \leftarrow(I-R A)^{v} v,
$$

the values of $v_{i}$ and $v_{j}$ should be close.

In Lean AMG, we generate $K$ Test Vectors (TVs). Each TV is the result of applying $v$ GS relaxation sweeps to $A x=0$, staring from randomly generated vectors $x^{(1)}, \ldots, x^{(K)} \in \mathbb{R}^{n}$. And we denote

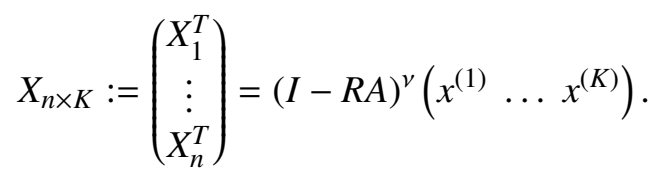

Here $X_{i}^{T}$ is the $i$-th row of $X_{n \times K}$. The strength function for the Lean AMG is then defined as follows

$$
s_{c}(i, j):=\frac{\left|\left(X_{i}, X_{j}\right)\right|^{2}}{\left(X_{i}, X_{i}\right)\left(X_{j}, X_{j}\right)}
$$

\subsection{Bibliographical notes}

Classical algorithms for determining strength of connection are found in (Brandt et al. 1982b, Stüben 1983, Brandt et al. 1985, Ruge and Stüben 1987, Briggs, Henson and McCormick 2000). The original measure of strength of connection given in (Ruge 1985, Mccormick and Ruge 1989, Brandt et al. 1985, Ruge 1983) is nonsymmetric, but for theoretical considerations, which only depend on the symmetrized smoothers, it suffices to use the slightly more restrictive, but symmetric versions of the strength of connection.

Some extension of these classical algorithms for defining strong connections are based on different measures for connectivity and distance such as measure of importance, and algebraic distance. Details are found in (Ruge and Stüben 1987) and (Trottenberg et al. 2001, Appendix A).

The strength of connection measures has had little theoretical backing in the past. The results developed in this section, such as local Poincaré inequalities and 
especially the strength functions 8.15 shows that such heuristics are reasonable and their choice can be motivated by theoretical results.

For aggregation AMG, typically, a symmetric strength of connection function as defined in (Vaněk et al. 1996b) is used. Some recent aggregation algorithms also define strength of connection based on sharp theoretical results and use the local two-level convergence rate (8.11) as a measure (Notay 2010, Napov and Notay 2012).

For aggregations based on matching (aggregates of size 2) the the "heavy edge" matching algorithms in (Karypis and Kumar 1998) corresponds to a strength of connection function selecting aggregates depending on the edge weight in the adjacency graph. Some recent works (Livne and Brandt 2012) use strength of connection functions based on the size of the entries in the Gramm matrix formed by a set of smoothed test vectors.

\section{Coarsening strategies}

Once the smoother is identified, the central task of an AMG method is to identify a sequence of coarse spaces, in functional terminology, or equivalently, in an algebraic setting, to identify a sequence of prolongation matrices. This procedure is known as a "coarsening" procedure. In this section, we will discuss how this coarsening procedure.

Roughly speaking, given the equation (1.1), namely $A u=f$, on a vector space $V$, the goal is to find a subspace $V_{c} \subset V$ such that the solution $u_{c} \in V_{c}$ of the following "coarsened" problem:

$$
A_{c} u_{c}=f_{c}, \quad A_{c}=\imath_{c}^{\prime} A \imath_{c}, f_{c}=\imath_{c}^{\prime} f
$$

would provide a good approximation to the original solution $u \in V$. More specifically, the solution of $u_{c}$ of 9.1) would provide good approximation to those "algebraically smooth" components of the error for which the given smoother does not converge well.

\subsection{Motivations}

In some sense, "coarsening" is done almost everywhere in numerical analysis. For example, the finite element equation (2.19) can be viewed as a coarsened equation of the original equation (2.3). In this case the finite element space $V_{h}$ is a coarsened subspace of $V=H_{0}^{1}(\Omega)$.

It is therefore informative for us to exam how a finite element space is constructed in general. While there are many different ways to construct finite element spaces, mathematically speaking, the most convenient approach is through the use of "degrees of freedom" which refers to a basis of a dual space. More specifically, in a finite element discretization, the finite element space $V_{h}$ is obtained by specifying the dual space $V_{h}^{\prime}$ (the so called space of degrees of freedom) first. For linear 
finite elements, $V_{h}^{\prime}=\left\{\psi_{i}: i=1: n_{h}\right\}$ is such that

$$
\psi_{i}(v)=v\left(x_{i}^{h}\right) .
$$

With such degrees of freedom (nodal evaluation), we then find a dual basis $\left\{\phi_{i}\right.$ : $\left.i=1: n_{h}\right\}$ which are piecewise linear functions such that

$$
\psi_{i}\left(\phi_{j}\right)=\delta_{i j}, \quad 1 \leq i, j \leq n .
$$

The finite element space $V_{h}$ is then defined by

$$
V_{h}=\operatorname{span}\left\{\phi_{i}: i=1: n_{h}\right\} .
$$

In fact, mathematically speaking, all existing finite element spaces $V_{h}$ can be obtained by first constructing $V_{h}$. This is the approach taken in the classical literature on finite element methods, c.f. (Ciarlet 2002).

Similar to finite element method, we will therefore focus on techniques for constructing a coarse space $V_{c} \subset V$ by first identifying its dual basis $V_{c}^{\prime}$. Such an approach is rather abstract, but it turns out to be more intrinsic, more general, and, in fact, more commonly used in AMG literature (implicitly).

It is interesting to note that we rarely use the word "coarsening" in the design of a geometric multigrid method. Instead, we use a "refinement" procedure to define a sequence of nested spaces. As an example, Figure 9.6 shows a uniformly refined triangular grid used for discretization of Poisson equation with with linear finite elements.

In AMG, we do not have the luxury of this hierarchy of spaces given by a geometric refinement. Instead, we carry out a reverse-engineering process of the refinement process, namely coarsening.
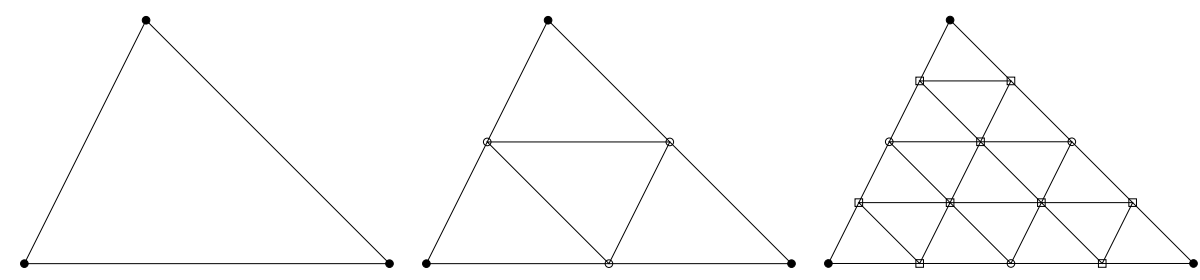

Figure 9.6. Regular refinement of a coarse grid element. •-coarsest level vertices, o-first level of refinement, $\square$-second level of refinement.

Conceivably, we can also use such an inverse-engineering process to recover the geometric multigrid method by starting from the finest geometric grid, at least for some special case. For example, if all triangles in the triangulation as shown in Figure 9.6 are of acute type and then the graph corresponding to the mesh is also an adjacency graph of the stiffness matrix. It is clear that the coarse grid vertices, i.e. the set $C$ (known from the refinement), is a maximal independent set (MIS) of vertices in the graph corresponding to the refined mesh. In the simplest case, 
the degrees of freedom associated with the coarse grid (the dual basis for $V_{c}$ ) precisely corresponds to the MIS. Such an observation is explored later in constructing algorithms in the framework of Classical AMG for a selection of coarse grid vertices via the MIS algorithm in $\$ 9.3 .1$. For further examples on relations between geometric AMG and GMG coarsening we refer to $\$ 10.2$.

The above reverse-engineering process for GMG gives some hint how a coarsening process needs to be done in AMG, but we need to study the process in a broader framework and more importantly we will use the degrees of freedom, namely basis of dual basis, to obtain coarse spaces. In the above GMG example, each grid point in the geometric grid corresponds exactly to one degree of freedom. But this is not always the case in applications.

\subsection{Basic approach}

By mimicking the construction of finite element space as described above, given a linear algebraic system of equation $A u=f$, we adapt a coarsening strategy consisting of the following steps:

1 We consider the adjacency graph $\mathcal{G}(A)$ of the coefficient matrix $A$. Based on certain strength function $s_{c}$ as described in $\$ 8$, we remove the weakly connected edge in $\mathcal{G}(A)$, namely drop certain entries in $A$ to form a filtered matrix $\tilde{A}$.

2 We carry out one of the following two:

(a) Classical AMG: Find an MIS of $\mathcal{G}(\tilde{A})$ to form the set of coarse vertices $C$. Then remove the rest, namely $\mathcal{V} \backslash \mathcal{C} \equiv \mathcal{F}$.

(b) Aggregation AMG: Agglomerate using some greedy algorithm: pick a point and agglomerate its neighbors and go from there.

3 We obtain a subset of d.o.f. obtained from the above steps, namely $\left(V^{\prime}\right)_{c}$

4 We use $\left(V^{\prime}\right)_{c}$ to define a high frequency space $V_{h f}=\left(V^{\prime}\right)_{c}^{(0)}$ by

$$
V_{h f}=\left(V^{\prime}\right)_{c}^{(0)}:=\left\{v \in V:\langle g, v\rangle=0, \quad \forall g \in\left(V^{\prime}\right)_{c}\right\}
$$

5 Find a tentative coarse space $W_{c}$ such that

$$
V=V_{h f} \oplus W_{c}
$$

6 Apply certain postprocess (such as smoothing) to $W_{c}$ to obtain $V_{c}$ :

$$
V_{c}=S W_{c}
$$

7 Using $V_{c}$, or equivalently the prolongation $P$, we form coarse matrix $A_{c}=$ $P^{T} A P$.

8 We then repeat the above steps to $A_{c}$ in place of $A$ until a desirable coarsest level is reached. 


\subsubsection{Construction of $\left(V^{\prime}\right)_{c}$}

Given $A \in \mathbb{R}^{n \times n}$ and the associated graph $\mathcal{G}=(\mathcal{V}, \mathcal{E})$, we proceed as follows:

1. Form the following two non-overlapping decompositions

$$
\mathcal{V}=C \cup \mathcal{F}, \quad C=\bigcup_{i=1}^{n_{c}} \mathcal{A}_{i}
$$

2. Identify $\left(V^{\prime}\right)_{c}=\operatorname{span}\left\{N_{i}: i=1: n_{c}\right\} \subset V^{\prime}$.

Here are three examples which will be discussed in detail in later sections (c.f. $\S 11$, $\$ 12$, and $\$ 13$.

Aggregation AMG : $\mathcal{F}=\emptyset$ and

$$
N_{i}(v)=\langle v\rangle_{\mathcal{A}_{i}} \equiv \frac{1}{\left|\mathcal{A}_{i}\right|} \sum_{j \in \mathcal{H}_{i}} \psi_{j}(v)=\frac{1}{\left|\mathcal{A}_{i}\right|} \sum_{j \in \mathcal{A}_{i}} v_{j}, \quad i=1: n_{c} .
$$

Classical AMG : $\mathcal{F} \neq \emptyset$ and

$$
\mathcal{A}_{i}=\left\{k_{i}\right\}, \quad N_{i}(v)=\psi_{k_{i}}(v)=v_{k_{i}} .
$$

In this case, $C$ usually consists of disconnected vertices.

Energy-min AMG : $\mathcal{F} \neq \emptyset$ and $\mathcal{A}_{i}$ are aggregates

$$
N_{i}(v)=\langle v\rangle_{\mathcal{A}_{i}} \equiv \frac{1}{\left|\mathcal{A}_{i}\right|} \sum_{j \in \mathcal{H}_{i}} \psi_{j}(v)=\frac{1}{\left|\mathcal{A}_{i}\right|} \sum_{j \in \mathcal{A}_{i}} v_{j}, \quad i=1: n_{c} .
$$

\subsubsection{Construction of $V_{c}$}

Given coarse grid degrees of freedoms $\left(V^{\prime}\right)_{c} \subset V^{\prime}$, we define $V_{h f}$ as in (9.2). The following lemma shows how to find a subspace $W_{c}$, a "pre-coarse space", such that $V=V_{h f} \oplus W_{c}$.

Lemma 9.1. If $\phi_{k, c}, k=1, \ldots, n_{c}$ are elements of $V$ such that the "Gramm" matrix

$$
G=\left(G_{k m}\right)=\left(N_{m}\left(\phi_{k, c}\right)\right)
$$

is nonsingular, then we have

$$
V=V_{h f} \oplus W_{c}, \quad W_{c}=\operatorname{span}\left\{\phi_{k, c}\right\}_{k=1}^{n_{c}} .
$$

Proof. We first show $W_{c} \cap V_{h f}=\{0\}$. In fact, if $v:=\sum_{k=1}^{n_{c}}(\tilde{v})_{k} \phi_{k, c} \in W_{c} \cap V_{h f}$, then we have

$$
0=N_{m}(v)=\sum_{k=1}^{n_{c}} N_{m}\left(\phi_{k, c}\right)=\sum_{k=1}^{n_{c}}(\tilde{v})_{k} G_{k m}, \quad m=1, \ldots, n_{c} .
$$

Hence,

$$
G \tilde{v}=0,
$$


and since by assumption, $G$ is nonsingular, we must have $v=0$. Therefore, $W_{c} \cap$ $V_{h f}=\{0\}$.

Next, for any $v \in V$, we define $w_{c} \in W_{c}$ as

$$
w_{c}=\sum_{k=1}^{n_{c}}\left(\widetilde{w}_{c}\right)_{k} \phi_{k, c}, \quad \widetilde{w}_{c}=G^{-1}\left(\begin{array}{c}
N_{1}(v) \\
\vdots \\
N_{n_{c}}(v) .
\end{array}\right) .
$$

It is immediate to check that

$$
N_{m}\left(v-w_{c}\right)=0, \quad m=1, \ldots n_{c} .
$$

This means that $\left(v-w_{c}\right) \in V_{h f}$. This proves that $v=w_{c}+(\underbrace{v-w_{c}}_{\in V_{h f}})$ and completes the proof.

The above lemma gives us a way to construct a subspace $W_{c}$ such that $V=$ $V_{h f} \oplus W_{c}$.

Lemma 9.2. If the coarse grid degrees of freedom are defined as

$$
N_{k}:=\sum_{j \in \mathcal{A}_{k}} \alpha_{j} \psi_{j}, \quad k=1, \ldots, n_{c},
$$

where $\sum_{j \in \mathcal{A}_{k}} \alpha_{j}=1$ and $\left\{\psi_{j}\right\}$ is dual basis of $\left\{\phi_{j}\right\}$. If $\left\{\phi_{k, c}: k=1, \ldots, n_{c}\right\}$ are elements of $V$ such that the "Gramm" matrix

$$
G=\left(N_{l}\left(\phi_{k, c}\right)\right)=I
$$

then, we have

$$
\phi_{k, c}=\sum_{j \in \mathcal{A}_{k}} \phi_{j}+v_{h f}, \quad v_{h f} \in V_{h f}
$$

Proof. We fix a $k$ and consider the subset $W_{k} \subset V$ such that

$$
W_{k}:=\left\{v \in V: N_{k}(v)=1 \text { and } N_{l}(v)=0, \forall l \neq k\right\}
$$

Pick any $v_{1}, v_{2} \in W_{k}$, we have

$$
N_{l}\left(v_{1}-v_{2}\right)=N_{l}\left(v_{1}\right)-N_{l}\left(v_{2}\right)=0, \quad \forall l=1, \ldots, n_{c},
$$

and this shows that $\left(v_{1}-v_{2}\right) \in V_{h f}$.

Furthermore, if we define $v_{k}^{c}=\sum_{j \in \mathcal{A}_{k}} \phi_{j}$, then

$$
N_{k}\left(v_{k}^{c}\right)=\sum_{j \in \mathcal{A}_{k}} \sum_{i \in \mathcal{A}_{k}} \alpha_{j}\left(\psi_{j}, \phi_{i}\right)=\sum_{j \in \mathcal{A}_{k}} \alpha_{j}=1 .
$$

and $N_{l}\left(v_{k}^{c}\right)=0$ for all $l \neq k$ since $\mathcal{A}_{k}$ and $\mathcal{A}_{l}$ have no overlap. We then have $v_{k}^{c} \in W_{k}$ and hence,

$$
W_{k}=\left\{v_{k}^{c}+v_{h f}: v_{h f} \in V_{h f}\right\}
$$


Next, we describe how one can construct the coarse space $V_{c}$ using $W_{c}$.

Lemma 9.3. Assume that $V=V_{h f} \oplus W_{c}$ and $\varphi_{1, c}, \ldots \varphi_{n_{c}, c}$ is a basis in $W_{c}$. Then $\phi_{k, c}=S \varphi_{k, c}, k=1, \ldots, n_{c}$ are linearly independent if $S: V \mapsto V$ satisfies one of the following conditions

1. $S$ maps a linear independent set in $W_{c}$ into a linear independent set in $V$.

2. $S$ is invertible;

3. $S=I-Q_{h f}$ where $Q_{h f}: V \mapsto V_{h f}$.

As a result, we have

$$
V=V_{h f} \oplus V_{c}, \quad V_{c}=\operatorname{span}\left\{S \varphi_{k, c}: k=1, \ldots, n_{c}\right\} .
$$

Proof. We only need to prove the third case as the first two cases are trivial. If $\phi_{k, c}$ were linearly dependent, there would be a linear combination of $\left\{\phi_{k, c}\right\}_{k=1}^{n_{c}}$ which vanishes. Equivalently, this means that there exists $w_{c} \in W_{c}$ such that $\left(I-Q_{h f}\right) w_{c}=$ 0 , which implies that $w_{c} \in V_{h f}$. Since $V_{f} \cap W_{c}=\{0\}$ we have that $w_{c}=0$ and the only vanishing linear combination in $\operatorname{span}\left\{\phi_{k, c}\right\}_{k=1}^{n_{c}}$ is the trivial one and this completes the proof.

\section{Remark 9.4.}

1. For smoothed aggregation, $S=I-\omega D^{-1} A$ for some properly chosen $\omega$ so that $S$ is nonsingular, or maps a special linearly independent set of vectors to linearly independent set of vectors (see $\$ 13$ ).

2. For classical AMG with ideal interpolation, $S=I-Q_{h f}$ and $Q_{h f}$ is $A$-othogonal projection (see $\$ 12$ ).

3. For classical AMG with standard interpolation, $S=I-Q_{h f}$ and $Q_{h f}$ is an approximation to the ideal interpolation (see $\$ 12$ ).

To give a summary of above discussions, the coarsening algorithms in AMG are methods for determining the coarse grid degrees of freedom (or coarse grid variables). Such algorithms are based on selecting degrees of freedom associated with subsets of vertices in the adjacency graph that corresponds to the matrix $A$ or to the strength matrix $S$ as is done in geometric coarsening, when the hierarchy of meshes or adjacency graphs is known.

\subsection{Two basic coarsening algorithms}

In the next two sections we present typical algorithms for finding the coarse grid degrees of freedom. Each such degree of freedom is associated with a vertex or a subset of a graph. Two types of algorithms are distinguished: Classical AMG algorithm pick coarse grid degrees of freedom that correspond to a maximal independent set of vertices in adjacency graph of the strength matrix; and aggregation based AMG algorithms which use splitting of adjacency graph of the strength matrix in connected subgraphs. 


\subsubsection{A maximal independent set (MIS) algorithm}

We present here a simple "greedy" maximal independent set (MIS) algorithm, which has been used in the Classical AMG algorithms to identify coarse grid degrees of freedom. Given adjacency graph of the strength matrix, the simple greedy MIS algorithm is as follows.

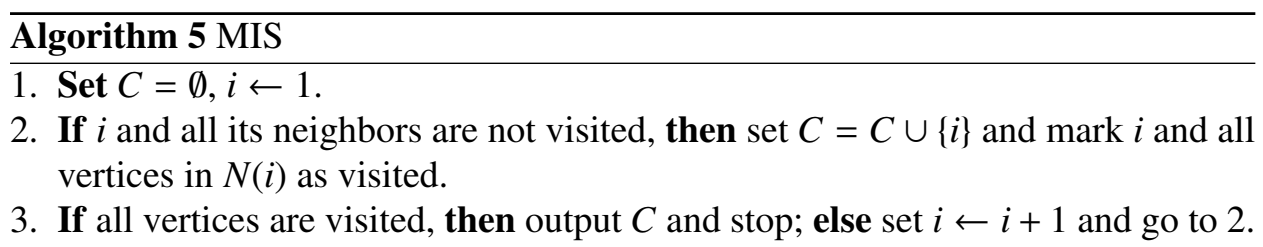

Remark 9.5. We note that the MIS Algorithm 5 recovers the geometric coarsening if the vertices are visited in an order so that coarse grid vertices are ordered first and all the connections in Figure 9.6 are strong. This is obvious, but, nevertheless, shows that the geometric coarsening can sometimes be recovered by an algebraic algorithm.

Let us point out that for finite element stiffness matrices obtained via adaptive refinement algorithms, the hierarchy of vertices is naturally included in the refinement procedure. For regular refinement such choice of MIS is illustrated in Figure 9.6 .

\subsubsection{An aggregation algorithm}

The class of algorithms, known as aggregation algorithms refer to splitting of adjacency graph of the strength matrix as a union of connected subgraphs. Let $\left\{\mathcal{V}_{k}\right\}_{k=1}^{n_{c}}$ be a non-overlapping splitting of the set of vertices

$$
\mathcal{V}=\cup_{k=1}^{n_{c}} \mathcal{V}_{k}, \quad \mathcal{V}_{j} \cap \mathcal{V}_{k}=\emptyset, \quad \text { for } \quad j \neq k .
$$

We then define

$$
\mathcal{E}_{k}=\left\{(l, m) \in \mathcal{E} \mid l \in \mathcal{V}_{k} \text { and } m \in \mathcal{V}_{k}\right\},
$$

to be the set of edges associated with $\mathcal{V}_{k}$.

An aggregation can be done in many different and some very sophisticated ways. In general all combinatorial graph partitioning algorithms can be used for aggregation. We, however, will not consider in details such algorithms, but rather, we provide here (Algorithm 6) the basic and most important example of greedy aggregation algorithm.

Such algorithm can be recursively applied to provide a multilevel hierarchy of aggregates. 
Algorithm 6 Greedy aggregation algorithm

Input: Graph $\mathcal{G}$ with $n$ vertices; Output: $\mathcal{V}=\cup_{k=1}^{n_{c}} \mathcal{V}_{k}$, and $\mathcal{V}_{k} \cap \mathcal{V}_{j}=\emptyset$ when $k \neq j$.

1. Set $n_{c}=0$ and for $k=1: n$ do:

a. If $k$ and all its neighbors have not been visited, then: (a) we set $n_{c}=n_{c}+1$; (b) label with $n_{c}$ the subgraph whose vertices are $k$ and the neighbors of $k$; and (c) mark $k$ and all its neighbors as visited.

b. If at least one neighbor of $k$ has been visited, we continue the loop over the vertices.

2. Since after this procedure there might be vertices which do not belong to any aggregate (but definitely have a neighboring aggregate), we add each such vertex to a neighboring aggregate and we pick the one which has minimal number of vertices in it.

3. The algorithm ends when all vertices are in a subset.

\subsubsection{Aggressive coarsening}

The extended strong connections and the corresponding strength operator are used to construct coarse spaces of smaller dimension. This procedure is also known as aggressive coarsening. We recall the definition of a path in the graph given in $\$ 7.1$ and all our considerations are on the adjacency graph $\mathcal{G}(S)$ of the strength matrix $S \in \mathbb{R}^{n \times n}$ defined in (8.4). Aggressive coarsening refers to selection of coarse grid vertices as independent set in the adjacency graph corresponding to the strength operator are at distances larger than 2 .

Definition 9.6. (Strong connection along a path) A vertex $i$ is said to strongly connect to a vertex $j$ along a path of length $l$ if there exits a path $\left(k_{0}, k_{1}, \ldots, k_{l}\right)$ in $\mathcal{G}(S)$, such that $k_{0}=i, k_{l}=j$, and $s_{c}\left(k_{m}, k_{m+1}\right) \geq \theta, m=0,1, \ldots, l-1$.

Next definition is related to the number of strongly-path-connected vertices.

Definition 9.7. $((m, l)$-strong connection) For given integers $m>0$ and $l>0$, a vertex $i$ is $(m, l)$-strongly connected to a vertex $j$ if and only if $i$ strongly connect to $j$ along at least $m$ paths of length $l$ (per Definition 9.6.

An aggressive coarsening algorithm generates a MIS using Algorithm 5 for the graph $\mathcal{G}_{m, l}=\left(\mathcal{V}, \mathcal{E}_{m, l}\right)$ with a set of vertices $\mathcal{V}=\left\{1, \ldots, n\right.$ and set of edges $\mathcal{E}_{m, l}$ defined as

$$
\mathcal{E}_{m, l}:=\{(i, j) \mid i \text { is }(m, l) \text { strongly connected to } j\}
$$

As is well known (Diestel 2010) $\left(S^{l}\right)_{i j}$ is nonzero if and only if there is a path of length $\leq l$ between $i$ and $j$. An aggressive coarsening exploits this property and is an algorithm which selects a set of coarse grid degrees of freedom corresponding to 
vertices in the graph which are at graph distance larger than $l$. It uses the adjacency graph $\mathcal{G}\left(S^{l}\right)$ of $S^{l}$ in place of $\mathcal{G}(S)$ in an aggregation or a MIS algorithm.

As an example, let us consider aggressive coarsening with $l=2$ and $m=1$. The set of coarse grid degrees of freedom is obtained by applying the standard MIS Algorithm 5 twice: (1) we find a MIS in $\mathcal{G}(S)$ and then obtain a set of coarse grid degrees of freedom $C$ (these are at graph distance at least 2); (2) Apply the MIS algorithm for a second time on the graph with vertices the $C$-points and edges between them given by the strength operator corresponding to $S^{2}$.

Similarly, for aggregation, an aggressive coarsening corresponds to applying the aggregation Algorithm 6 recursively, or applying it directly to the graph corresponding to $S^{l}$ for a given $l$.

\subsection{Adaptive coarsening for classical $A M G$}

An adaptive coarsening algorithm is an algorithm which adaptively chooses the coarse grid degrees of freedom based on a given definition of strength function based on the smoother in a two grid algorithm. One example of adaptive coarsening follows from the classical compatible relaxation introduced by A. Brandt. The algorithm takes as input a smoother which leaves the coarse grid variables invariant and only smooths the components in the algebraic high-frequency space $V_{\mathrm{hf}}$.

A typical adaptive coarsening algorithm follows the steps given below:

Step 0 Set $k=0$ and choose $\left(V^{\prime}\right)_{k, c} \subset V^{\prime}$, for example, using the MIS or aggregation method introduced in $\$ 9.3$.

Step 1 Define $V_{k, f}$ as the subspace of $V$ which is annihilated by the functionals in $\left(V^{\prime}\right)_{k, c}$, namely

$$
V_{k, f}=\left\{v \in V:(g, v)=0, \forall g \in\left(V^{\prime}\right)_{k, c}\right\} .
$$

Step 2 Let $l_{k, f}: V_{k, f} \mapsto V$ be the natural inclusion operator and compute an estimate $\rho_{k, f}$ of the norm of the smoother on $V_{k, f}$. Below, $R_{k, f}$ could be the restriction of the smoother $R$ on $V_{k, f}$, or more generally, any relaxation on $V_{k, f}$.

$$
\rho_{k, f} \approx \sup _{v \in V_{k, f}} \frac{\left\|\left(I-\iota_{k, f} R_{k, f} \iota_{k, f}^{\prime} A\right) v\right\|_{A}^{2}}{\|v\|_{A}^{2}} .
$$

Step 3 Given a threshold $\delta_{f}>0$, if $\rho_{k, f}>\delta_{f}$, we set $k=k+1$, add more functionals to $\left(V^{\prime}\right)_{c}$ and go to Step 1. Otherwise, we set $\left(V^{\prime}\right)_{c}=\left(V^{\prime}\right)_{k, c}$, and accordingly $V_{f}=V_{k, f}$ and stop the iteration.

In Step 3, if the stopping criteria is not satisfied, we need to enrich the space $\left(V^{\prime}\right)_{c}$. by extending the set. One example of doing so is introduced in compatible relaxation method by extending the set $C$ using the following procedure:

First we randomly choose a vector $v^{0} \in V_{k, f}$, and form

$$
v=\left(I-\iota_{k, f} R_{k, f} \iota_{k, f}^{\prime} A\right)^{v} v^{0} \text { for some } v \geq 1 .
$$


Then, with a given threshold $\theta \in(0,1)$, we let

$$
C_{0}^{1}=\left\{i \in \mathcal{F}:\left|v_{i}\right|>\theta \max _{k}\left|v_{k}\right|\right\} \text {. }
$$

and

$$
C_{1}=C_{0} \cup \operatorname{MIS}\left(C_{0}^{1}\right) .
$$

Finally, we update $C \leftarrow \mathcal{C}_{1}$ and we proceed with the next CR iteration.

As is clear from the algorithm outlined above, we can use any of the definitions of strength of connections to obtain $\left(V^{\prime}\right)_{0, c}$ at Step 0. When $\rho_{k, f}>\delta_{f}$, it means that $C$ obtained from the strength function $s_{0}$ is not satisfactory, namely too coarse. This either means that the threshold for $s_{0}$ is too small, or $s_{0}$ itself is not satisfactory. We could still use $s_{0}$ but with a smaller threshold to obtain a $C_{0}^{\prime}$ which is bigger than $C_{0}$, but not necessarily contain $C_{0}$. For example, if we initially use the $(m, l)$ strong connection defined in Definition 9.7, $C$ can be extended by increasing the value of $m$ or decreasing the value of $l$. This approach may not be computationally efficient. A more effective approach, as it is used in compatible relaxation, is to find candidates for additional $C$-points by examining the following filtered matrix:

$$
A^{(1)}=\left\{a_{i j}: i, j \in \mathcal{F}_{0}\right\},
$$

where $\mathcal{F}_{0}=\Omega \backslash C_{0}$, to get the set of coarse grid degrees of freedom $C_{0}^{1}$ and add them to $C_{0}$ to extend the size of $C$.

\subsection{AGMG coarsening: a pairwise aggregation}

AGMG uses the strength function defined in (8.13) to form aggregations such that the local convergence rate (c.f (6.14) in $\$ 6$ ) on each aggregate is bounded by a given threshold. The main idea of the algorithm can be explained as follows:

It first splits the index set $\Omega$ into aggregates each of which has at most two elements, namely

$$
\Omega=\bigcup_{j} \mathcal{A}_{j}^{(0)}, \quad \mathcal{A}_{i}^{(0)} \bigcap \mathcal{A}_{j}^{(0)}=\emptyset, \quad \text { and }\left|\mathcal{A}_{j}^{(0)}\right| \leq 2 .
$$

This process is done by a greedy algorithm. At each step, the algorithm finds the pair $G=\{i, j\}$ for which the strength function defined in (8.14) is maximal.

Using these pairs as aggregates $\left\{\mathcal{A}_{j}^{(0)}\right\}$, we form the unsmoothed aggregation prolongation $P$, which is piecewise constant with respect to the aggregates and $P$ has orthogonal columns.

We denote $A^{(0)}:=A$ and $P^{(0)}:=P$. Then $A^{(1)}:=\left(P^{(0)}\right)^{T} A^{(0)} P^{(0)}$. We then apply pairwise aggregation algorithm on $A^{(1)}$ and find larger aggregates $\left\{\mathcal{A}_{j}^{(1)}\right\}$. Each $\mathcal{A}_{j}^{(1)}$ is union of two pairs in $\left\{\mathcal{A}_{j}^{(0)}\right\}$ which minimize the strength function defined in (8.13). Then we obtain $P^{(1)}$ and $A^{(2)}$. Applying this procedure recursively, we obtain the final aggregates $\mathcal{A}_{j}$ each of which is a union of several pairs in $\left\{\mathcal{A}_{j}^{(0)}\right\}$. 
The pairwise aggregation strategy aims to find the aggregates on which the Poncaré constant $\mu_{j}\left(V_{j}^{c}\right)^{-1}$, which is introduced in our abstract framework by (6.14), is bounded . And as it is stated in the abstract convergence theorem, bounding $\mu_{j}\left(V_{j}^{c}\right)^{-1}$ will bound the convergence rate of the AMG method.

\subsection{Bibliographical notes}

The coarsening strategies used in AMG are basically two types: the first one uses strength of connection to define a "strength" graph, and then performs greedy aggregation or MIS algorithms and the other is a based on algorithms such as Compatible Relaxation coarsening which uses the smoother to detect slow to converge components. These are heuristic approaches, which work well on a certain class of problems, but rarely have a theoretical justification of their efficiency as AMG splitting algorithms.

Classical algorithms for selection of the coarse grid degrees of freedom are found in (Brandt et al. 1982b, Stüben 1983, Brandt et al. 1985, Ruge and Stüben 1987), and the MG the tutorial (Briggs et al. 2000).

Parallel coarse-grid selection algorithms are found in (Sterck, Yang and Heys 2006) and, in combination with scalable interpolation algorithms in (De Sterck, Falgout, Nolting and Yang 2008). Coarsening using information about discretization, i.e. AMGe, are given in (Jones and Vassilevski 2001, Brezina, Cleary, Falgout, Henson, Jones, Manteuffel, McCormick and Ruge 2001). Spectral AMGe coarsening considered in detail in (Chartier et al. 2003). Many of the "upscaling" and related techniques in homogenization (c.f. (Efendiev, Hou and Wu 2000, Hou, $\mathrm{Wu}$ and Cai 1999)), resemble the coarsening procedures introduced in the classical and modern AMG literature.

More sophisticated maximal independent set (MIS) algorithm for selection of coarse grid degrees of freedom, using different measures for connectivity and distance in the graph corresponding to $A$ such are found in (Ruge and Stüben 1987) and (Trottenberg et al. 2001, Appendix A). Most of these algorithms are refinements of the greedy algorithm given in this section. For parallel versions we refer to (Luby 1986), (Cleary, Falgout, Henson and Jones 1998), (Sterck et al. 2006) for specific details on parallel and parallel randomized MIS algorithms. Other coarsening schemes that are also suitable for parallel implementation are the coupled and decoupled coarsening schemes (Yang 2006, Henson and Yang 2002).

Regarding the aggregation coarsening methods we refer to (Vakhutinsky, Dudkin and Ryvkin 1979), (Blaheta 1986), and Marek (Marek 1991) a for earlier work on such methods. The greedy aggregation algorithm presented here is found in (Vaněk et al. 1996b). A special class of aggregation coarsening method based on matching were first employed by Kumar and Karypis for fast graph partitioning (Karypis and Kumar 1998) and later used in several of the AMG methods. One example is the AGMG algorithm described in $\$ 9.5$ and found in (Napov and Notay 2012, Notay 2012). The algorithm given in (Kim, Xu and Zikatanov 2003) also uses such coars- 
ening approach.Special matching techniques which optimize matrix invariants are used in (D'Ambra and Vassilevski 2014, D'Ambra and Vassilevski 2013). a The Compatible Relaxation (CR) algorithm, first introduced in (Brandt 2000a) and further investigated in (Livne 2004), (Falgout and Vassilevski 2004), and (Brannick and Falgout 2010), is a device that reduces the role of the strength of connection to only define initial set of coarse grid degrees of freedom and then use the smoother to select additional degrees of freedom. Other coarse-fine degrees of freedom partitioning algorithms are considered in (MacLachlan and Saad 2007) from both classical as well as compatible relaxation point of view. A somewhat different adaptive coarsening algorithms are the aggregation algorithms which aggregates vertices together based on a local measure for two-level convergence (Notay 2010, Napov and Notay 2012, Livne and Brandt 2012).

\section{GMG, AMG and a geometry-based AMG}

Historically, the algebraic multigrid, AMG, method was motivated by the geometric multigrid, GMG, method. In this section, we will give exploration on the relationship between these two types of methods.

\subsection{Geometric multigrid method}

We begin out discussion for a simple 1D model problem, namely (2.1) for $d=$ $1, \Omega=(0,1)$ and $\alpha \equiv 1$ with zero Dirichlet boundary condition. For any integer $N$, we consider a uniform grid, denoted by $\mathcal{T}_{h}$, of the interval $[0,1]$ as follows:

$$
0=x_{0}<x_{1}<\cdots<x_{N+1}=1, \quad x_{j}=\frac{j}{N+1}(j=0: N+1) .
$$

This partition consists of uniform subintervals with the length $h=\frac{1}{N+1}$, i.e., $\mathcal{T}_{h}=$ $\bigcup_{i}\left\{\tau_{i}\right\}$ where $\tau_{i}=\left(x_{i-1}, x_{i}\right)$ for $d=1$. Such a uniform partition is shown in Figure 10.7

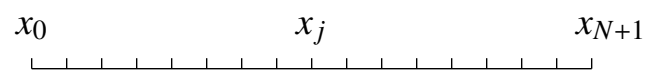

Figure 10.7. One-dimensional uniform grid

We define a linear finite element space associated with the partition $\mathcal{T}_{h}$

$V_{h}=\left\{v: v\right.$ is continuous and piecewise linear w.r.t. $\left.\mathcal{T}_{h}, v(0)=v(1)=0\right\} .(10.2)$

Let $V_{h}=V_{h}$. Recall from previous section, the finite element approximation of our model problem is then $u_{h} \in V_{h}$ satisfying (2.19). We introduce the operator: $A_{h}: V_{h} \mapsto V_{h}$ such that

$$
\left(A_{h} v_{h}, w_{h}\right)=a\left(v_{h}, w_{h}\right), \quad v_{h}, w_{h} \in V_{h} .
$$


Then the finite element solution $u_{h}$ satisfies

$$
A_{h} u_{h}=f_{h}
$$

where $f_{h} \in V_{h}$ is the $L^{2}$ projection of $f:\left(f_{h}, v_{h}\right)=\left(f, v_{h}\right), \quad v_{h} \in V_{h}$.

To describe a geometric multigrid algorithm, we need to have a multiple level of grids, say $\mathcal{T}_{k}$ with $k=1: J$ and $\mathcal{T}_{J}=\mathcal{T}_{h}$ being the finest mesh. One simple definition of the grid points in $\mathcal{T}_{k}$ is as follows:

$$
x_{i}^{k}=\frac{i}{2^{k}}, \quad i=0,1,2, \cdots, N_{k}+1, k=1,2, \cdots, J,
$$

where $N_{k}=2^{k}-1$. Note that $\mathcal{T}_{k}$ can be viewed as being obtained by adding midpoints of the subintervals in $\mathcal{T}_{k-1}$. For each $k$ the set of above nodes will be denoted by $\mathcal{N}_{k}$.

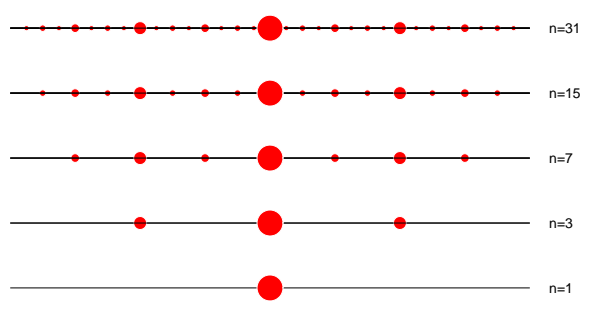

Figure 10.8. Multiple grids in one dimension

For $k=1: J$, similar to finite element space $V_{h}$ defined as in (10.2), we define finite element space $V_{k}$ associated with the grid $\mathcal{T}_{k}$ to obtain a nested sequence of finite element spaces as follows:

$$
V_{1} \subset \ldots \subset V_{k} \subset \ldots \subset V_{J}
$$

The classic V-cycle geometric multigrid method simply applies Algorithm 4 recursively with the following setting:

$1 V_{k}=V_{k}$;

$2 A_{k}: V_{k} \mapsto V_{k}$ defined by

$$
\left(A_{k} u_{k}, v_{k}\right)=a\left(u_{k}, v_{k}\right), \quad u_{k}, v_{k} \in V_{k}
$$

$3 l_{k-1}^{k}: V_{k-1} \mapsto V_{k}$ is the inclusion operator;

$4 R_{k}: V_{k} \mapsto V_{k}$ corresponding a smoother such as Gauss-Seidel method.

\section{Algebraic setting}

The equation 10.3 may be called the operator form of the finite element equation. To get an equation in terms of vectors and matrix, we use the nodal basis functions 
for $V_{h}$

$$
\phi_{i}(x)=\left\{\begin{array}{cl}
\frac{x-x_{i-1}}{h}, & x \in\left[x_{i-1}, x_{i}\right] ; \\
\frac{x_{i+1}-x}{h}, & x \in\left[x_{i}, x_{i+1}\right] ; \\
0 & \text { elsewhere }
\end{array}\right.
$$

On each level, similarly as (10.5), we can introduce a set of nodal basis functions, denoted by $\left\{\phi_{i}^{(k)}: i=1: N_{k}\right\}$, for finite element space $V_{k}$.

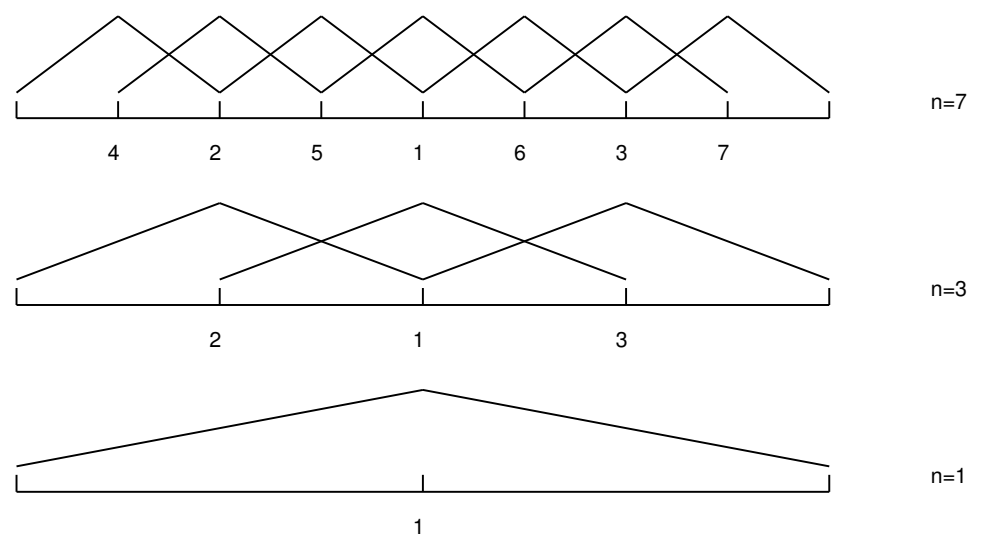

Figure 10.9. 1D nodal basis functions on each level

Each $v \in V_{k}$ can be uniquely written as the following linear combination of the basis functions

$$
v=\xi_{1} \phi_{1}^{(k)}+\xi_{2} \phi_{2}^{(k)}+\cdots+\xi_{N_{k}} \phi_{N_{k}}^{(k)}
$$

This gives an isomorphism from $V_{k}$ to $\mathbb{R}^{N_{k}}$, which maps $v \in V_{k}$ to $\mu \in \mathbb{R}^{N_{k}}$ as following

$$
v=\xi_{1} \phi_{1}^{(k)}+\xi_{2} \phi_{2}^{(k)}+\cdots+\xi_{N_{k}} \phi_{N_{k}}^{(k)} \longrightarrow \mu=\left(\begin{array}{c}
\xi_{1} \\
\xi_{2} \\
\vdots \\
\xi_{N_{k}}
\end{array}\right) .
$$

$\mu$ is called matrix representation of $v$. Recall from the discussion earlier in $\$ 2.2$. giving a basis of $V_{h}$ defined in (10.5), the (2.19) is equivalent to the linear system equations in 2.20).

We introduce auxiliary space $V_{k}:=\mathbb{R}^{N_{k}}$. 
The transition operator $P_{k}^{k+1}$ from $V_{k}$ to $V_{k+1}$ is a matrix in $\mathbb{R}^{N_{k+1} \times N_{k}}$ and satisfies

$$
\left(\phi_{1}^{(k)} \cdots \phi_{N_{k}}^{(k)}\right)=\left(\phi_{1}^{(k+1)} \cdots \phi_{N_{k+1}}^{(k+1)}\right) l_{k}^{k+1} .
$$

For the special 1D problem we are now considering, we have for $k=1,2, \ldots, J-1$

$$
\phi_{j}^{(k)}=\frac{1}{2} \phi_{2 j-1}^{(k+1)}+\phi_{2 j}^{(k+1)}+\frac{1}{2} \phi_{2 j+1}^{(k+1)}
$$

The matrix which encodes this relation is

$$
P_{k}^{k+1}=\left(\begin{array}{ccccc}
\frac{1}{2} & & & & \\
1 & & & & \\
\frac{1}{2} & \frac{1}{2} & & & \\
& 1 & & & \\
& \frac{1}{2} & \frac{1}{2} & & \\
& 1 & & \\
& \frac{1}{2} & & \\
& & \ddots & \frac{1}{2} \\
& & & 1 \\
& & & \frac{1}{2}
\end{array}\right) .
$$

The classical $V$-cycle AMG method following from simply applying Algorithm 4 recursively with the following setting:

1. $V_{k}=\mathbb{R}^{N_{k}}$

2. $A_{k} \in \mathbb{R}^{N_{k} \times N_{k}}: V_{k} \mapsto V_{k}$ defined by

$$
\left(A_{k}\right)_{i j}=a\left(\phi_{i}^{(k)}, \phi_{j}^{(k)}\right), \quad 1 \leq i, j \leq N_{k}
$$

3. $P_{k-1}^{k}: V_{k-1} \mapsto V_{k}$ a matrix in $\mathbb{R}^{N_{k+1} \times N_{k}}$ defined by 10.8;

4. $R_{k}: V_{k} \mapsto V_{k}$ corresponding a smoother such as Gauss-Seidel method.

A similar multigrid algorithm can be obtained for problems in 2D and 3D as long as we have a multiple level of grids and the corresponding finite element spaces on each level.

\subsection{Obtaining AMG from $G M G$}

The first barrier of extending GMG to AMG is the geometric information used in GMG. But a close inspection of the GMG reveals that a GMG method only depends on the following 2 major ingredients:

1. The stiffness matrix corresponding to the finest grid;

2. The prolongation matrix on each level. 
Once all the prolongation matrices are given, the stiffness matrices on all coarser levels are given by

$$
A_{k}=P_{k}^{T} A P_{k}, \quad k=J-1, J-2, \ldots 1, \quad P_{k}=\prod_{j=k}^{J-1} P_{j}^{j+1} .
$$

Of course, a smoother is also needed on each level, but its definition can be considered, for the moment purely algebraic.

As the stiffness matrix on the finest grid is always available in any given application, the only thing left is the prolongation matrices. We will now use the example of linear finite element method to discuss about the relationship between the prolongation matrix and geometric information. Two observations are most relevant:

Observation 1 The prolongation matrix only depends on the natural graph associated with the underlying grid, but not on the coordinates of grid points.

Observation 2 The graph of the underlying grid is very close to the adjacency graph of the stiffness matrix.

Based on the above discussions, roughly speaking, we can essentially recover a geometric multigrid method for the stiffness matrix corresponding to the continuous linear finite element discretization of Laplace equation by only using the algebraic and graph information provided by the stiffness matrix.

1. Form the adjacency graph $\mathcal{G}(A)$ of the stiffness matrix $A$;

2. Coarsen $\mathcal{G}(A)$.

As an illustrative example, let us consider the stiffness matrices corresponding to a discretization of the Laplace equation on a square domain with bilinear elements. It is well known, (Ciarlet 2002), that the stiffness matrix in this case is the same as the scaled matrix for the 9-point finite difference stencil (2.18). The corresponding adjacency graph, shown on the right in Figure 10.10 is denser (has more edges) than the mesh graph shown on the left in Figure 10.10. The set of its edges includes the diagonals of each of the squares forming the mesh. For the construction of the prolongation/interpolation matrix we recall that the prolongation matrix gives the coefficients of the expansion of a coarse grid basis function on a grid of size $2 h$ in terms of the finer grid basis on a grid of size $h$. Locally this matrix looks as follows:

$$
\left[\left(P_{2 h}^{h}\right)^{T}\right]_{i}=\left(\begin{array}{ccc}
\frac{1}{4} & \frac{1}{2} & \frac{1}{4} \\
\frac{1}{2} & 1 & \frac{1}{2} \\
\frac{1}{4} & \frac{1}{2} & \frac{1}{4}
\end{array}\right) .
$$

This matrix is often dubbed as "prolongation stencil" and it shows in a compact form the coefficients in the expansion of a coarse grid basis function. In the center we have the coefficient 1 , in front of the fine grid basis function associated with a 

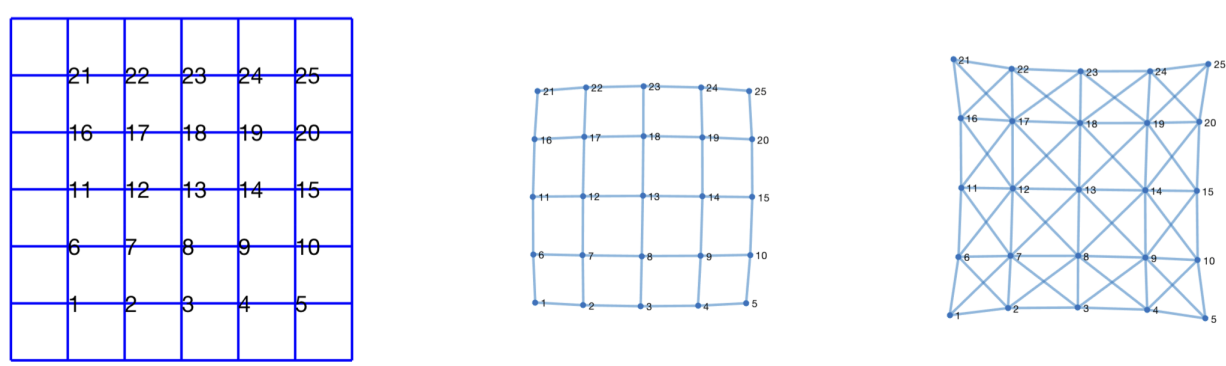

Figure 10.10. A $6 \times 6$ uniform grid (left), graph of the matrix corresponding to the 5-point finite difference stencil (middle) and graph of the matrix corresponding to the 9-point finite difference stencil (right)

coarse grid vertex. The rest of the entries correspond to the coefficients in front of the fine grid basis functions in the expansion.

On regularly refined, triangular grids we have an analogous situation. We refer to $\$ 9$ for details on the selection of coarse grid degrees of freedom in this case. Prolongation and restriction matrices only depend on the topological structure of this graph. Similar observations led to the development of the AMG: if the geometric coordinates are unknown, different avenues for constructing coarse spaces are needed, leading to different variants of AMG algorithms.

\subsection{Obtaining $G M G$ from $A M G$}

In this section, we use the unified theory in $\$ 6$ to obtain GMG from AMG. The main ingredients needed are spaces $V_{j}$, operators $\Pi_{j}, A_{j}, D_{j}$ and coarse spaces $V_{j}^{c}$.

We now consider constructing a two-level geometric multigrid method for 2.1 (or the variabtional formulation (2.3)). Suppose we have two grids: a fine grid $\mathcal{T}_{h}$ and a coarse grid $\mathcal{T}_{H}$. On each grid, we define a linear finite element space $V_{h}$ and $V_{H}$ with nodal basis functions $\left\{\phi_{j}^{h}\right\}$ and $\left\{\phi_{j}^{H}\right\}$ respectively and we consider the following partition of the domain $\Omega$ :

$$
\bar{\Omega}=\bigcup_{j=1}^{J} \bar{\Omega}_{j}, \text { with } \bar{\Omega}_{j}=\operatorname{supp}\left(\phi_{j}^{H}\right) .
$$

We then define $V_{j}$ as

$$
V_{j}:=\left\{\chi_{j} v: v \in V_{h}\right\},
$$

where $\chi_{j}$ is the characteristic function of $\Omega_{j}$. We note that $V_{j}$ is not a subspace of $H^{1}(\Omega)$. The operator $\Pi_{j}: V_{j} \mapsto V_{h}$ is defined in the following way:

$$
\Pi_{j} v_{j}:=I_{h}\left(\phi_{j}^{H} v_{j}\right), \quad \forall v_{j} \in V_{j}, \quad j=1, \ldots, J,
$$

where $I_{h}$ is the nodal interpolation operator on the fine grid. Here we note that $\phi_{j}^{H} v_{j}$ 
is continuous in $\Omega$. We notice that, by definition, $\phi_{j}^{H} \chi_{j}=\phi_{j}^{H}$ on $\Omega$ which implies the identities

$$
\sum_{j=1}^{J} \Pi_{j} \chi_{j} v=I_{h}\left(\sum_{j=1}^{J} \phi_{j}^{H} \chi_{j} v\right)=I_{h}\left(\sum_{j=1}^{J} \phi_{j}^{H} v\right)=I_{h}(v)=v,
$$

and hence

$$
\sum_{j=1}^{J} \Pi_{j} \chi_{j}=\mathrm{Id}
$$

The operator $A_{j}: V_{j} \mapsto V_{j}^{\prime}$ is the local restriction of the bilinear form $a(\cdot, \cdot)$, namely,

$$
\left(A_{j} u_{j}, v_{j}\right):=a\left(u_{j}, v_{j}\right)_{\Omega_{j}}=\int_{\Omega_{j}} \alpha(x) \nabla u_{j} \cdot \nabla v_{j}, \quad u_{j}, v_{j} \in V_{j}
$$

The following estimate tells us that $A_{j}$ satisfies 6.11) with decomposition $v=$ $\sum_{j=1}^{J} \Pi_{j} v_{j}$ where $v_{j}=\chi_{j} v$ :

$$
\sum_{j=1}^{m_{c}}\left\|v_{j}\right\|_{A_{j}}^{2}=\sum_{j=1}^{m_{c}} a(v, v)_{\Omega_{j}} \leq C_{1}\|v\|_{A}^{2}
$$

where $C_{1}$ depends on the number of overlaps of $\Omega_{j}$.

We choose $D: V \mapsto V^{\prime}$ using the fine grid basis functions as follows

$$
\left(D \phi_{i}^{h}, \phi_{j}^{h}\right):=a\left(\phi_{i}^{h}, \phi_{j}^{h}\right) \delta_{i j}, \quad 1 \leq i, j \leq n,
$$

and

$$
(D u, v):=\sum_{j=1}^{n} a\left(\phi_{j}^{h}, \phi_{j}^{h}\right) u_{j} v_{j}, \quad u, v \in V .
$$

Note that $(D \cdot, \cdot)$ is an inner product on $V$ which induces a norm $\|\cdot\|_{D}$.

In the above definition, the matrix representation of $D$ is the diagonal of the matrix representation of $A$. In a similar way, we can define $D_{j}: V_{j} \mapsto V_{j}^{\prime}$ using the basis functions for $V_{j}$.

By a simple scaling argument, we obtain

$$
\|v\|_{0}^{2} \equiv h^{d}\|v\|_{\ell^{2}}^{2} .
$$

From (10.17), using inverse inequality, we then have

$$
\|v\|_{D}^{2} \lesssim h^{-2} \sum_{j=1}^{n}\left\|\phi_{j}^{h}\right\|_{0}^{2} v_{j}^{2} \equiv h^{d-2}\|v\|_{\ell^{2}}^{2} \equiv h^{-2}\|v\|_{0}^{2} .
$$

We also have

$$
\left\|I_{h} v\right\|_{0}^{2} \equiv h^{d}\left\|I_{h} v\right\|_{\ell^{2}}^{2}=h^{d}\|v\|_{l^{2}}^{2} \equiv\|v\|_{0}^{2} .
$$


Assumption 6.10 then can then be verified by the following

$$
\begin{aligned}
\left\|\sum_{j=1}^{J} \Pi_{j} v_{j}\right\|_{D}^{2} & \lesssim h^{-2}\left\|\sum_{j=1}^{J} \Pi_{j} v_{j}\right\|_{0}^{2}=h^{-2}\left\|I_{h}\left(\sum_{j=1}^{J} \phi_{j}^{H} v_{j}\right)\right\|_{0}^{2} \\
& \equiv h^{-2}\left\|\sum_{j=1}^{J} \phi_{j}^{H} v_{j}\right\|_{0}^{2}=h^{-2} \sum_{i, j=1}^{J} \int_{\Omega} \phi_{i}^{H} v_{i} \phi_{j}^{H} v_{j} \\
& \leq h^{-2} \sum_{i, j=1}^{J}\left\|\phi_{i}^{H}\right\|_{\infty}\left\|\phi_{j}^{H}\right\|_{\infty} \int_{\Omega} v_{i} v_{j} \leq h^{-2} \sum_{i, j=1}^{J} \int_{\Omega_{i} \cap \Omega_{j}} v_{i} v_{j} \\
& \leq C_{o} \sum_{j=1}^{J} h^{-2} \int_{\Omega_{j}}\left|v_{j}\right|^{2} \lesssim C_{o} \sum_{j=1}^{J}\left\|v_{j}\right\|_{D_{j}}^{2} .
\end{aligned}
$$

By the definition of $A_{j}$, the kernel of $A_{j}$ consists of all constant functions in $V_{j}$ and we choose $V_{j}^{c}$ to be the one dimensional space of constant functions on $\Omega_{j}$. Then

$$
\mu_{j}\left(V_{j}^{c}\right)=\lambda_{j}^{(2)},
$$

where $\lambda_{j}^{(2)}$ is the second smallest eigenvalue of the operator $D_{j}^{-1} A_{j}$.

The global coarse space $V_{c}$ is defined as in (6.13). Note that in this case, it is easy to show by the definition that the coarse space $V_{c}$ constructed by 6.13 ) is in fact identical to $V_{H}$, namely

$$
V_{c}=\operatorname{span}\left\{\phi_{j}^{H}: j=1, \ldots, J\right\} .
$$

By Theorem 6.9, the converges rate of this two-level geometric multigrid method depends on the $\min _{j}\left(\lambda_{j}^{(2)}\right)$. If the Poincaré inequality is true for each $V_{j}$, namely,

$$
\inf _{v_{c} \in V_{j}^{c}}\left\|v-v_{c}\right\|_{D_{j}}^{2} \leq c_{j}\|v\|_{A_{j}}^{2}, \quad \forall v \in V_{j},
$$

with $c_{j}$ to be a constant, then the two-level geometry multigrid method converges uniformly.

\subsection{Spectral AMGe: a geometry-based $A M G$}

We now consider the element based AMG approaches, and also define the ingredients needed to fit these methods in the general results in $\$ 6$. The AMGe methods are less algebraic as they assume an underlying grid and use element local stiffness matrices to define interpolation operators. In the AMGe setting, it is assumed that we know a decomposition of the $n \times n$ matrix $A$,

$$
A=\sum_{\tau \in \mathcal{T}} \tilde{A}_{\tau}
$$


where, $\mathcal{T}$ is the set of finite elements used to discretize the problem and for each element $\tau \in \mathcal{T}$, and $\tilde{A}_{\tau}$ is the zero-extension of the local stiffness matrix $A_{\tau}$ on $\tau$ (which is symmetric positive semi-definite).

To define $V_{j}, D_{j}, A_{j}$ and $\Pi_{j}$ in $\$ 6$, corresponding to AMGe, we partition the domain $\Omega$ into disjoint subdomains, $\Omega_{1}, \ldots, \Omega_{J}$. Each subdomain is an agglomerate of elements, and $\bar{\Omega}=\bigcup_{j=1}^{J} \bar{\Omega}_{j}$. For each subdomain $\Omega_{j}$, we introduce the cutoff operator $\chi_{j}: V \mapsto V_{j}$ whose action on $v \in V$ is defined by

$$
\left(\chi_{j} v\right)(x):= \begin{cases}v(x), & \text { if } x \in \bar{\Omega}_{j}, \\ 0, & \text { if } x \notin \bar{\Omega}_{j} .\end{cases}
$$

Then we define the space $V_{j}$ by

$$
V_{j}:=\chi_{j} V
$$

$A_{j}$ is defined by summing up all the associated stiffness matrices for elements in $\Omega_{j}$, namely,

$$
A_{j}:=\sum_{\tau \subset \Omega_{j}} \tilde{A}_{\tau}
$$

Clearly, $A_{j}$ is symmetric positive semi-definite. It is easy to verify that 6.11) holds. In fact, we have the following equations

$$
\sum_{j=1}^{J}\left\|\chi_{j} v\right\|_{A_{j}}^{2}=\sum_{j=1}^{J} a(v, v)_{\Omega_{j}}=\sum_{\tau \in \mathcal{T}} a(v, v)_{\tau}=\|v\|_{A}^{2} .
$$

If we denote the diagonal of $A$ by $D$, then $D_{j}$ is a diagonal matrix defined following

$$
\left[D_{j}\right]_{i i}:= \begin{cases}D_{i i}, & \text { if } i \in \Omega_{j}, \\ 0, & \text { if } i \notin \Omega_{j} .\end{cases}
$$

The operator $\Pi_{j}$ is provided by diagonal matrices defined as

$$
\left[\Pi_{j}\right]_{i i}:= \begin{cases}{\left[A_{j}\right]_{i i} /[A]_{i i},} & \text { if } i \in \Omega_{j}, \\ 0, & \text { if } i \notin \Omega_{j} .\end{cases}
$$

Note that $\left[\Pi_{j}\right]_{i i}=1$ if $i$ is an inner point of $\Omega_{j} .6 .10$ is verified in Lemma 12.4

On each local space $V_{j}$, spectral AMGe chooses locally the "best" coarse space $V_{j}^{c}$ which is the subspace spanned by eigenvectors of $D_{j}^{-1} A_{j}$ belonging to its $m_{j}^{c}$ smallest eigenvalues. The global coarse space is then defined by (6.13).

By the abstract convergence theorem (Theorem 5.2), the convergence rate of two level spectral AMGe depends on minimum of each $m_{j}^{c}+1$ smallest eigenvalue of $D_{j}^{-1} A_{j}$ on $V_{j}$, more precisely,

$$
\|E\|_{A} \leq 1-\frac{\mu_{c}}{C_{p, 1} C_{p, 2}},
$$


with $\mu_{c}=\min _{1 \leq j \leq J} \mu_{m_{j}^{c}+1}^{(j)}$.

\subsection{Bibliographical notes}

The main ideas of GMG method were first demonstrated by pioneering works of Fedorenko (Fedorenko 1961, Fedorenko 1964), and Bahvalov (Bahvalov 1966). Similar ideas, using group relaxation methods, can be traced back to the works of Southwell in the 1940s (Southwell 1940, Southwell 1946). The first description of truly multi-grid method is found in the seminal work by Brandt (Brandt 1973). Further developments in multilevel methods are by Brandt (Brandt 1977) and also by Hackbusch (Hackbusch 1977, Hackbusch 1978). These works have drawn a lot of attention from computational mathematics and engineering community. Advances of convergence analysis of multigrid methods have been made by Nicolaides (Nicolaides 1975, Nicolaides 1977), Bank and Dupont (Bank and Dupont 1980) Braess and Hackbush (Braess and Hackbusch 1983), Bramble and Pasciak (Bramble and Pasciak 1987), Bramble, Pasciak and Xu (Bramble et al. 1990, Bramble, Pasciak and Xu 1991a), Bramble, Pasciak, Wang and Xu(Bramble et al. 1991c), $\mathrm{Xu}(\mathrm{Xu} 1992)$.

BoxMG (Dendy 1982, Dendy 1983) is a method that uses geometrically refined grids and defines interpolation using algebraic techniques. We refer to (Dendy 1982, Dendy 1983), (de Zeeuw 1990) for details and aslo to (MacLachlan, Moulton and Chartier 2012) for results on the equivalence between BoxMG and Classical AMG.

The element based AMG approaches, which are less algebraic as they assume an underlying grid and use element stiffness matrices to define interpolation operators. Such methods include plain AMGe, element-free AMGe, spectral AMGe, and spectral agglomerate and are developed to improve AMG robustness for finite element problems element based AMG. We refer to results and discussions on different flavors of AMGe to (Jones and Vassilevski 2001, Brezina et al. 2001, Henson and Vassilevski 2001, Brezina, Falgout, MacLachlan, Manteuffel, McCormick and Ruge 2006c).

\section{Energy-min AMG}

Here we consider the energy minimization algorithms for construction of coarse spaces. While this is not historically the first AMG approach to coarsening, we focus on this technique first, as it can be used to motivate most other AMG algorithms.

\subsection{Energy-minimization versus trace-minimization}

In the next Theorem we add a constraint to Theorem 5.7 and give a relation between the optimal coarse space $V_{c}^{\mathrm{opt}}$ and the energy minimization. We refer to $\$ 5.2$ for the definition of $P^{\text {opt }}$ and $X_{\eta}$. 
Theorem 11.1. (Trace-minimization theorem) Given $\eta>0$, let $\mathcal{Z}_{\eta}$ be defined as

$$
\mathcal{Z}_{\eta}=\left\{P \in \mathbb{R}^{n \times n_{c}}:(P v, P v)_{\bar{R}^{-1}} \geq \eta(v, v), \quad v \in \mathbb{R}^{n_{c}} \text { and } P \mathbf{1}=\sqrt{n_{c} \eta} \zeta_{1}\right\}
$$

Then, $P \in \arg \min _{Q \in \mathcal{Z}_{\eta}} \operatorname{trace}\left(Q^{T} A Q\right)$ if

$$
P \in \mathcal{Z}_{\eta} \text { and } \operatorname{Range}(P)=\operatorname{Range}\left(P^{\text {opt }}\right) .
$$

Let $\hat{P}=\bar{R}^{-\frac{1}{2}} P$ and define

$$
\mathcal{Y}_{\eta}=\left\{P \in \mathbb{R}^{n \times n_{c}}:(P v, P v) \geq \eta(v, v), v \in \mathbb{R}^{n_{c}} \text { and } P 1=\sqrt{n_{c} \eta} \hat{\zeta}_{1}\right\},
$$

where $\hat{\zeta}_{j}$ is the eigenvector corresponding to the $j$-th smallest eigenvalue of $\bar{R}^{\frac{1}{2}} A \bar{R}^{\frac{1}{2}}$. It is clear that $\bar{R}^{\frac{1}{2}} A \bar{R}^{\frac{1}{2}}$ and $\bar{R} A$ have the same spectrum. Theorem 11.1 can be written as

Theorem 11.2. Given $\eta>0$, let $\mathcal{Y}_{\eta}$ be defined as in (11.2). Then,

$$
P \in \underset{Q \in \mathcal{Y}_{\eta}}{\arg \min } \operatorname{trace}\left(Q^{T} \bar{R}^{\frac{1}{2}} A \bar{R}^{\frac{1}{2}} Q\right) \text { if } P \in \mathcal{Y}_{\eta} \text { and } \operatorname{Range}(P)=\operatorname{Range}\left(P^{\mathrm{opt}}\right) .
$$

Suppose now we have a bilinear form $a(\cdot, \cdot)$ on $V$ which is symmetric, positive semi-definite, and an inner product $(\cdot, \cdot)_{\bar{R}^{-1}}$ on $V$. Here, for example, the operator $\bar{R}$ is the scaled parallel (resp. successive) subspace correction method corresponding to the splitting of $V$ as

$$
V=\sum_{i=1}^{n} \operatorname{span}\left\{\phi_{i}\right\} .
$$

In practice, $\bar{R}$ can be a symmetrization of any $A$-norm convergent smoother on $V$. Here, for simplicity, we choose $\bar{R}=D^{-1}$.

We now consider a finite element space $V$ with basis functions $\left\{\phi_{j}: j=1: n\right\}$. Let $P=\left(p_{i j}\right) \in \mathbb{R}^{n \times n_{c}}$ be such that

$$
\left(\phi_{1}^{c}, \ldots, \phi_{n_{c}}^{c}\right)=\left(\hat{\phi}_{1}, \ldots, \hat{\phi}_{n}\right) P, \quad \hat{\phi}_{j}=\phi_{j} /\left\|\phi_{j}\right\|_{A}, \quad j=1: n .
$$

Denote by $\left\{\left(\mu_{j}, \zeta_{j}\right)\right\}$ the eigen-pairs of $\bar{R} A$, and $\hat{\zeta}_{j}=\zeta_{j} /\left\|\zeta_{j}\right\|_{A}$. We then define

$$
X_{\eta}=\left\{\left(\phi_{1}^{c}, \ldots, \phi_{n_{c}}^{c}\right): \text { it satisfies (11.3) with } P \in \mathcal{Y}_{\eta}\right\} \text {. }
$$

We consider the minimization problem

$$
\min _{\left(\phi_{1}^{c}, \ldots, \phi_{n_{c}}^{c}\right) \in X_{\eta}} \sum_{j=1}^{n_{c}}\left\|\phi_{j}^{c}\right\|_{A}^{2}
$$


We notice that

$$
\begin{aligned}
\sum_{j=1}^{n_{c}}\left\|\phi_{j}^{c}\right\|_{A}^{2} & =\sum_{j=1}^{n_{c}} a\left(\phi_{j}^{c}, \phi_{j}^{c}\right)=\sum_{j=1}^{n_{c}} a\left(\sum_{k=1}^{n} p_{k j} \hat{\phi}_{k}, \sum_{l=1}^{n} p_{l j} \hat{\phi}_{l}\right) \\
& =\sum_{j=1}^{n_{c}} \sum_{k=1}^{n} \sum_{l=1}^{n} p_{k j} a\left(\hat{\phi}_{k}, \hat{\phi}_{l}\right) p_{l j}=\operatorname{trace}\left(P^{T} D^{-\frac{1}{2}} \tilde{A} D^{-\frac{1}{2}} P\right) .
\end{aligned}
$$

Then Theorem 11.1 implies that

$$
\operatorname{span}\left\{\zeta_{j}, 1 \leq j \leq n_{c}\right\}=\operatorname{span}\left\{\phi_{j}^{0}, 1 \leq j \leq n_{c}\right\},
$$

where

$$
\left(\phi_{1}^{0}, \ldots, \phi_{n_{c}}^{0}\right) \in \underset{\left(\phi_{1}^{c}, \ldots, \phi_{n_{c}}^{c}\right) \in X_{\eta}}{\arg \min } \sum_{j=1}^{n_{c}}\left\|\phi_{j}^{c}\right\|_{A}^{2} .
$$

In the next section, we use the functional setting and provide details on the design of energy minimizing basis.

\subsection{Energy minimization basis for $A M G$ and Schwarz methods}

The discussion above motivates the computation of an energy minimizing basis as the solution of a global optimization problem with constraint. This could be of concern regarding the efficiency of the proposed approach. As we show later in this section, however, this is not a concern because the optimization problem is well conditioned and can be solved efficiently. We also show below that that the basis functions solving the energy minimization problem are locally harmonic within each coarse grid "element". Such property of the energy minimizing basis suggests that various "harmonic extension" techniques, used to define coarse spaces in multigrid method (see (Chan, Xu and Zikatanov 1998, Brezina et al. 2001, Jones and Vassilevski 2001)) are very closely related to the energy minimization algorithms. This property also suggests that the energy minimizing basis may also be used for numerical homogenization for problems having a multiscale nature (see (Efendiev et al. 2000, Hou et al. 1999)).

We start our description with a given set of subdomains $\Omega_{i}$ with the property that none of the subdomains is fully contained in the union of the rest of them. More precisely, we have,

$$
\Omega=\bigcup_{i=1}^{n_{c}} \Omega_{i} \text { and } \bar{\Omega}_{i} \bigcap\left(\bigcup_{j \neq i} \Omega_{j}\right)^{c} \neq \emptyset,
$$

where the superscript $c$ is the standard set-complement. Equivalently, in pure algebraic setting, when there is no function space in the back ground, we may set up $\Omega_{i}$ as subset of vertices of the adjacency graph corresponding to a matrix $A$ ). The aim is to construct basis functions $\left\{\phi_{i}^{H}\right\}_{i=1}^{n_{c}}$ that are in $\mathcal{X}_{\eta}$ with the following additional 
restrictions:

$$
\operatorname{supp}\left(\phi_{i}^{H}\right) \subset \bar{\Omega}_{i}, \quad 1 \leq i \leq n_{c} .
$$

We want the basis functions to have a total minimal energy among all such functions, namely $\left\{\phi_{i}^{H}\right\}_{i=1}^{J}$ is the minimizer of:

$$
\min \sum_{i=1}^{n_{c}}\left\|\psi_{i}\right\|_{A}^{2} \quad \text { subject to } \quad \psi_{i} \in V_{i} \text { and }\left(\psi_{1}, \ldots, \psi_{n_{c}}\right) \in \mathcal{X}_{\eta} \text {. }
$$

Here

$$
V_{i}=\left\{v \in V_{h}: \operatorname{supp}(v) \subset \bar{\Omega}_{i}\right\}, \quad 1 \leq i \leq n_{c} .
$$

Thanks to (11.8, the decomposition 11.11 holds, namely

$$
V=\sum_{i=1}^{n_{c}} V_{i}
$$

Remark 11.3. In AMG, the minimization problem (11.9) written in terms of the prolongation matrices is as follows: Find $P \in \mathbb{R}^{n \times n_{c}}$ such that

$$
P=\underset{Y \in \mathbb{R}^{n \times n_{c}}}{\arg \min } \mathcal{F}(Y), \quad Y \mathbf{1}_{n_{c}}=\mathbf{1}_{n}, \quad \mathcal{F}(Y)=\operatorname{trace}\left(Y^{T} A Y\right) .
$$

In terms of vectors, local support means that few non-zeroes per column (or per row) are allowed in $P$. We note that the functions $\left\{\phi_{i}^{H}\right\}$ satisfying the properties mentioned above are linearly independent due to the second assumption in (11.8) and the constraint in 11.12). This linear independence is equivalent to assuming that $P$ is a full $\operatorname{rank}$ matrix (i.e. $\operatorname{rank}(P)=n_{c}$ ).

By the assumption in (11.8), for each $j$, there exists $k \in \Omega_{j}$ such that $k \notin \Omega_{i}$ for all $i \neq j$. We define

$$
\mathcal{A}_{j}=\left\{k \in \Omega_{j}: k \notin \Omega_{i}, i \neq j\right\}, \quad j=1: n_{c} .
$$

Then $\mathcal{A}_{j} \cap \mathcal{A}_{i}=\emptyset$ if $i \neq j$. We then define

$$
C=\bigcup_{j=1}^{n_{C}} \mathcal{A}_{j} \text { and } \mathcal{F}=\Omega \backslash C .
$$

We define $N_{j} \in V^{\prime}$ as follows

$$
N_{j}(v)=\frac{1}{\left|\mathcal{A}_{j}\right|} \sum_{i \in \mathcal{A}_{j}} \psi_{i}(v)=\frac{1}{\left|\mathcal{A}_{j}\right|} \sum_{i \in \mathcal{F}_{j}} v_{i}, \quad j=1: n_{c} .
$$

Clearly, $\left\{N_{j}\right\}_{j=1}^{n_{c}}$ are linearly independent, and if $\operatorname{supp}(v) \subset \Omega_{j}$, then $N_{i}(v)=0$ for all $i \neq j$.

We define $\left(V^{\prime}\right)_{c} \subset V^{\prime}$

$$
\left(V^{\prime}\right)_{c}=\operatorname{span}\left\{N_{j}: j=1: n_{c}\right\},
$$


and $V_{h f} \subset V$

$$
V_{h f}=\left\{v \in V:(g, v)=0, \forall g \in\left(V^{\prime}\right)_{c}\right\}
$$

If $\left\{\varphi_{j}\right\}_{j=1}^{n_{c}}$ satisfy

$$
\operatorname{supp}\left(\varphi_{j}\right) \subset \Omega_{j} \text { and } \sum_{j=1}^{n_{c}} \varphi_{j}=1,
$$

then we have the "Gramm" matrix $G=\left(G_{i j}\right)=\left(N_{j}\left(\varphi_{i}\right)\right)=I$. By Lemma 9.1, we have

$$
V=V_{h f} \oplus W_{c}, \quad W_{c}=\operatorname{span}\left\{\varphi_{j}: j=1: n_{c}\right\} .
$$

Let us first introduce some notation. We define the restriction $A_{i}$ of $A$ on each subspace $V_{i}$ as

$$
\left(A_{i} u_{i}, v_{i}\right)=\left(A u_{i}, v_{i}\right), \quad \forall u_{i}, v_{i} \in V_{i}, \quad i=1: n_{c} .
$$

Let $Q_{i}: V^{\prime} \mapsto V_{i}^{\prime}$ be a projection defined as the adjoint of the natural inclusion $V_{i} \subset V$ :

$$
\left\langle Q_{i} u^{\prime}, v_{i}\right\rangle=\left\langle u^{\prime}, v_{i}\right\rangle \quad \forall v_{i} \in V_{i}, \quad u^{\prime} \in V^{\prime} .
$$

We now define the following PSC type preconditioner (c.f. 4.17)

$$
B=\sum_{i=1}^{n_{c}} A_{i}^{-1} \iota_{i}^{\prime}=\sum_{i=1}^{n_{c}} l_{i} A_{i}^{-1} \iota_{i}^{\prime} .
$$

Thanks to (11.8), it is easy to see that the operator $T: V \mapsto V$ is an isomorphism.

We are now in a position to state and prove the first result in this section.

Theorem 11.4. The minimization problem (11.9) has a unique solution which is given by

$$
\phi_{i}^{H}=A_{i}^{-1} Q_{i} B^{-1} 1
$$

satisfying

$$
\operatorname{supp}\left(\phi_{i}^{H}\right) \subset \Omega_{i}
$$

Proof. This results actually follows directly from Theorem 4.4 with $v=1$. Let us give a different proof below. then is obtained by finding the critical point of the following quadratic functional:

$$
L=\sum_{i=1}^{n_{c}}\left(\frac{1}{2}\left\|\phi_{i}\right\|_{A}^{2}-\left\langle\lambda, \phi_{i}\right\rangle .\right)
$$

Differentiating this functional gives that

$$
\left[\partial_{\phi_{i}} L\right] \xi_{i}=\left(A \phi_{i}, \xi_{i}\right)-\left\langle\lambda, \xi_{i}\right\rangle, \quad \xi_{i} \in V_{i} .
$$

Hence the the $i$-th component of the critical point $\left(\phi_{i}^{H}\right)$ is given by

$$
\left(\phi_{i}, \xi_{i}\right)_{A}=\left\langle\lambda, \xi_{i}\right\rangle, \quad \forall \xi_{i} \in V_{i}, \quad i=1: n_{c} .
$$




\section{ENERGY-MIN AMG}

From the above equations we obtain that

$$
\phi_{i}^{H}=A_{i}^{-1} Q_{i} \lambda
$$

Summing up leads to:

$$
\lambda=B^{-1} 1
$$

This gives a derivation of (11.15).

It is obvious that this unique critical point $\left(\phi_{i}^{H}\right)$ is indeed the unique global minimizer of (11.9) that has a convex objective functional and a convex constraint.

We now show that the constructed basis functions are locally discrete A-harmonic. We say that a function $w \in V$ is discrete a-harmonic on a subdomain $D$ if

$$
(w, v)_{A}=0 \text {, for all } v \in V_{h, 0}(D) \equiv\left\{v \in V_{h}: \operatorname{supp}(v) \subset \bar{D}\right\} .
$$

This property requires defining the "subdomains" $D$ on which it holds. Below, we introduce such subdomains in terms of function spaces. Matrix/vector representations of the considerations below are easy to write. To define an analogue of coarse grid elements (an analogue to a finite element coarse grid), we first consider the set of all the points in $\Omega$ that are interior to all $\Omega_{i}$ 's:

$$
\omega_{0}=\left(\bigcup_{i=1}^{n_{c}} \partial \Omega_{i}\right)^{c} \bigcap \Omega .
$$

Given $x \in \omega_{0}$, define the following function with values in the subsets of $\left\{1, \ldots, n_{c}\right\}$ which is the set of indices of subdomains $\Omega_{i}$ that contain $x$ :

$$
I(x)=\left\{i: x \in \Omega_{i}\right\} .
$$

To rule out any ambiguity we shall assume that for any $x \in \omega_{0}$ the set $I(x)$ is ordered in ascending order. We then define

$$
K_{x}=\left\{y \in \omega_{0}: I(y)=I(x)\right\} .
$$

Namely $K_{x}$ is the intersection of all $\Omega_{i}$ that contain $x$ (see Figure 11.11).

The following simple proposition will lead us to an appropriate definition of coarse grid elements.

Proposition 11.5. For the sets $K_{x}$ defined in (11.18) we have

(a) $K_{x}=K_{y}, \Leftrightarrow I(x)=I(y)$.

(b) Either $K_{x} \cap K_{y}=\emptyset$ or $K_{x}=K_{y}, x \in \omega_{0}, y \in \omega_{0}$.

(c) There are a finite number $m_{H}$ of different sets $K_{x}, x \in \omega_{0}$.

Proof. The $(\Rightarrow)$ direction in (a) follows from the fact that $x \in K_{x}=K_{y}$, and hence $I(x)=I(y)$. The other direction follows from the definition of $K_{x, y}$.

To prove (b) let us assume that there exists $z \in \omega_{0}$, such that $z \in K_{x}$ and $z \in K_{y}$. The definition of $K_{x}$ and $K_{y}$ then gives that $I(x)=I(y)=I(z)$. By (a), $K_{x}=K_{y}$. This proves (b). 
The conclusion (c) follow directly from (b).

Let $\mathcal{T}_{H}$ denote the finite collection of $m_{H}$ sets in (c) from the above Proposition 11.5. We have

$$
\omega_{0}=\bigcup_{x \in \omega_{0}} K_{x}=\bigcup_{K \in \mathcal{T}_{H}} K
$$

As it is obvious that $\bar{\omega}_{0}=\bar{\Omega}$,

$$
\bar{\Omega}=\bar{\omega}_{0}=\overline{\bigcup_{K \in \mathcal{T}_{H}} K}=\bigcup_{K \in \mathcal{T}_{H}} \bar{K} .
$$

This means that the collection of $\mathcal{T}_{H}$ forms a non-overlapping partition of $\Omega$. Each element in $\mathcal{T}_{H}$ will be called a coarse grid element.

Remark 11.6. It is tempting to show how these macroelements look on an unstructured grid, and in Fig 11.11, we have depicted three such supports together with their intersection. But let us point out that an essential feature of the technique we present here is that the coarse elements need not be defined it explicitly and they might have quite complicated shape.
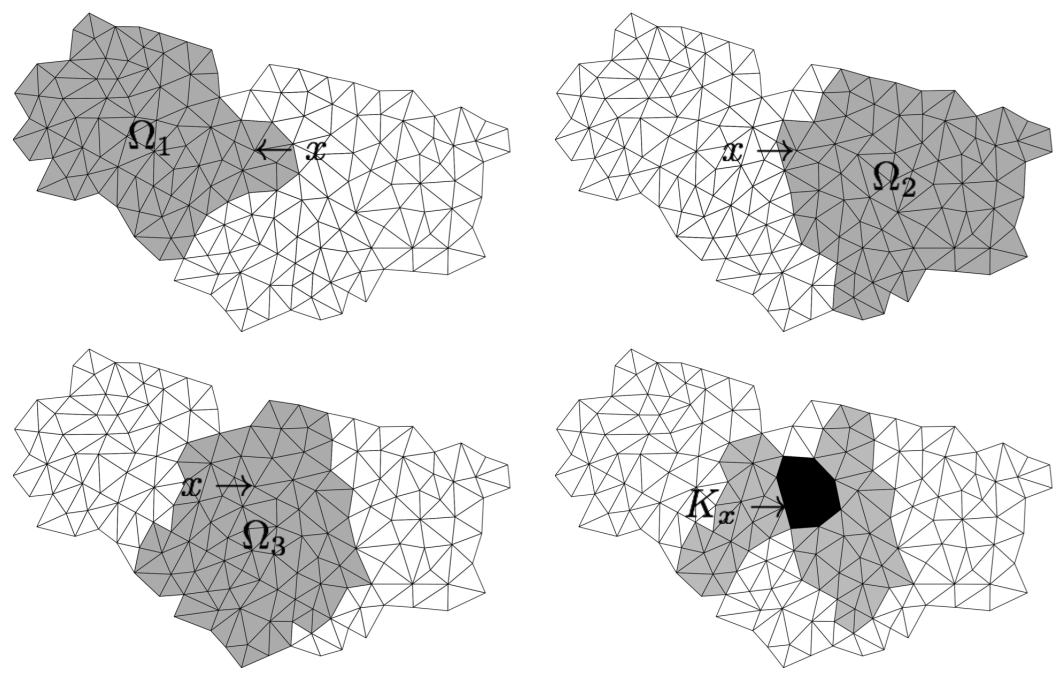

Figure 11.11. A piece of triangular grid and supports of three basis functions. On the right bottom picture, the intersections are plotted. The darker colored domain correspond to a coarse element and is intersection of all three supports. The lighter shaded domains are intersections of two supports and the white area corresponds to no intersection.

Lemma 11.7. Let $\lambda=B^{-1} 1$. Assume that for each coarse element $K \in \mathcal{T}_{H}$ as 
defined above, we have $\left(1, w_{K}\right)_{A}=0$ for all $w_{K}$ supported in $K$. Then

$$
\langle\lambda, \xi\rangle=0, \text { for all } \xi \in V_{h, 0}(K) .
$$

Proof. By definition $K=K_{y}$ for some $y \in \Omega$. Thus

$$
V_{h, 0}(K)=\bigcap_{i \in I(y)} V_{i} \text { and } \sum_{i \in I(y)} \phi_{i}^{H}(x)=1, x \in K .
$$

Thus, by (11.16, we have

$$
\left(\phi_{i}, \xi\right)_{A}=\langle\lambda, \xi\rangle, \quad \forall \xi \in V_{h, 0}(K)
$$

and

$$
\langle\lambda, \xi\rangle=\sum_{i \in I(y)}\left(\phi_{i}, \xi\right)_{A}=(1, \xi)_{A}=0 .
$$

The desired result then follows.

When the coarsening corresponds to a geometric multigrid and uniform refinement, the lemma shows that $\lambda=B^{-1} 1 \in V^{\prime}$ is a discrete edge $\delta$-function with respect to the coarse elements (namely $\lambda$ is supported around $\partial K$ ). Figure 11.12 is an example profile of $\lambda$ and a basis function $\Phi_{i}^{H}$.
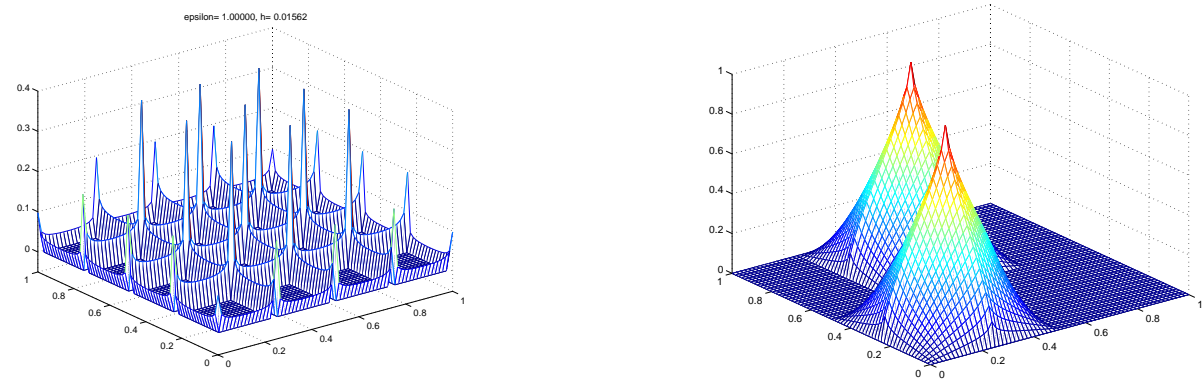

Figure 11.12. The profile of $\lambda=B^{-1} \mathbf{1}$ (left) and a typical basis function $\phi_{i}^{H}$ (right)

Combining the above result with the identity (11.16), we immediately obtain our second main result in this section.

Theorem 11.8. Each basis function $\phi_{i}^{H}$ is discrete a-harmonic on each coarse element $K \in \mathcal{T}_{H}$, namely

$$
\left(\phi_{i}^{H}, v\right)_{A}=0, \quad v \in V_{h, 0}(K) .
$$

In one space dimension $(d=1)$, the above result is rather trivial and it is in fact already contained in (Wan, Chan and Smith 1999/00). In this case, the basis function $\left(\phi_{i}^{H}\right)$ is analogous to the generalized finite element basis function in Babuška and Osborn (Babuška and Osborn 1983).

The local harmonic properties in all aforementioned literature are obtained by construction from local element boundaries. It is interesting to note that the energy 


\subsection{Convergence of energy minimization $A M G$}

minimizing basis studied here is a result of a more global construction and the local harmonic properties is a by-product from the construction.

\subsection{Convergence of energy minimization $A M G$}

We present a proof for the two-level convergence of AMG based on energy minimization.

We define cut off operators $\chi_{j}$ on the subdomains $\Omega_{j}$ introduced in 11.8 as following

$$
\left(\chi_{j} v\right)(x):= \begin{cases}v(x), & \text { if } x \in \bar{\Omega}_{j}, \\ 0, & \text { if } x \notin \bar{\Omega}_{j} .\end{cases}
$$

Then we define spaces $W_{j}$ by

$$
W_{j}:=\chi_{j} V=\chi_{j} v,: v \in V .
$$

And it is easy to verify that

$$
\left\{\chi_{j} \phi_{i}: \operatorname{supp} \phi_{i} \cap \Omega_{j} \neq \emptyset\right\}
$$

forms a basis of $W_{j}$.

The operator $\Pi_{j}: W_{j} \mapsto V$ is defined as in (10.14) with $\phi_{j}^{H}$ being the solution of the minimization problem $(11.9)(\sqrt{11.15})$ in Theorem 11.4$)$.

We then have that

$$
\sum_{j=1}^{n_{c}} \Pi_{j} \chi_{j}=\mathrm{Id}
$$

In fact, we have

$$
\sum_{j=1}^{n_{c}} \phi_{j}^{H}(x)=\sum_{j: x \in \Omega_{j}} \phi_{j}(x)=\sum_{j \in I(x)} \phi_{j}(x)=1,
$$

which implies the identity

$$
\sum_{j=1}^{n_{c}} \Pi_{j} \chi_{j} v=I_{h}\left(\sum_{j=1}^{n_{c}} \phi_{j}^{H} \chi_{j} v\right)=I_{h}(v)=v, \text { and hence } \sum_{j=1}^{n_{c}} \Pi_{j} \chi_{j}=\mathrm{Id} .
$$

The operator $A_{j}: W_{j} \mapsto W_{j}^{\prime}$ is the local restriction of the bilinear form $a(\cdot, \cdot)$ as in (10.15).

6.11) is satisfied with decomposition $v=\sum_{j=1}^{n_{c}} \Pi_{j} v_{j}$ where $v_{j}=\chi_{j} v \in W_{j}$ :

$$
\sum_{j=1}^{m_{c}}\left\|v_{j}\right\|_{A_{j}}^{2}=\sum_{j=1}^{m_{c}} a(v, v)_{\Omega_{j}} \leq C_{o}\|v\|_{A}^{2}
$$

where $C_{o}$ depends on the number of overlaps of $\Omega_{j}$. 
We choose $D: V \mapsto V^{\prime}$ using the fine grid basis functions as following

$$
\left(D \phi_{i}^{h}, \phi_{j}^{h}\right):=\left(A \phi_{i}^{h}, \phi_{j}^{h}\right) \delta_{i j}, \quad 1 \leq i, j \leq n .
$$

Notice that, in the above definition, the matrix representation of $D$ is the diagonal of the matrix representation of $A$. In a similar way, we can define $D_{j}: W_{j} \mapsto W_{j}^{\prime}$ using basis functions of $W_{j}$ defined in (11.21), and the matrix representation of $D_{j}$ is the diagonal of the matrix representation of $A_{j}$. It is well known that

$$
\|v\|_{D}^{2} \equiv h^{-2}\|v\|_{0}^{2}, \forall v \in V, \text { and }\left\|v_{j}\right\|_{D_{j}}^{2} \equiv h^{-2}\left\|v_{j}\right\|_{0}^{2}, \forall v_{j} \in W_{j} .
$$

6.10) can be verified by the following

$$
\begin{aligned}
\left\|\sum_{j=1}^{n_{c}} \Pi_{j} v_{j}\right\|_{D}^{2} & \lesssim h^{-2} \int_{\Omega}\left(\sum_{j=1}^{n_{c}} \phi_{j}^{H} v_{j}\right)^{2}=h^{-2} \sum_{i, j=1}^{n_{c}} \int_{\Omega} \phi_{i}^{H} v_{i} \phi_{j}^{H} v_{j} \\
& \leq h^{-2} \sum_{i, j=1}^{n_{c}}\left\|\phi_{i}\right\|_{\infty}\left\|\phi_{j}\right\|_{\infty} \int_{\Omega} v_{i} v_{j} \\
& \leq h^{-2}\left(\max _{1 \leq j \leq n_{c}}\left\|\phi_{j}\right\|_{\infty}\right)^{2} \sum_{i, j=1}^{n_{c}} \int_{\Omega_{i} \cap \Omega_{j}} v_{i} v_{j} \\
& \leq C_{o}\left(\max _{1 \leq j \leq n_{c}}\left\|\phi_{j}\right\|_{\infty}\right)^{2} \sum_{j=1}^{n_{c}} h^{-2} \int_{\Omega_{j}}\left|v_{j}\right|^{2} \\
& \lesssim C_{o}\left(\max _{1 \leq j \leq n_{c}}\left\|\phi_{j}\right\|_{\infty}\right)^{2} \sum_{j=1}^{n_{c}}\left\|v_{j}\right\|_{D_{j}}^{2} .
\end{aligned}
$$

We choose local coarse spaces $W_{j}^{c} \subset W_{j}$ to be the space of constant functions on $\bar{\Omega}_{j}$. Then the global coarse space $V_{c}$ is defined as

$$
V_{c}:=\sum_{j=1}^{n_{c}} \Pi_{j} W_{j}^{c} .
$$

In fact, for this case, $V_{c}$ is the subspace spanned by $\left\{\phi_{j}^{H}\right\}$ :

$$
V_{c}=\operatorname{span}\left\{\phi_{j}^{H}: j=1, \ldots, n_{c}\right\} .
$$

We choose the subdomain $\Omega_{j}$ in a way so that the Poincare inequality holds

$$
\inf _{v_{c} \in W_{j}^{c}}\left\|v-v_{c}\right\|_{0}^{2} \leq c d_{j}^{2}|v|_{1}^{2}
$$

where $d_{j}$ is the diameter of $\Omega_{j}$, and $c$ is a constant independent of the mesh size and the size of $\Omega_{j}$. Since

$$
\|v\|_{D_{j}}^{2} \equiv h^{-2}\|v\|_{0}^{2}, \quad\|v\|_{A_{j}}^{2} \equiv|v|_{1}^{2},
$$




\subsection{Bibliographical notes}

We have

$$
\inf _{v_{c} \in W_{j}^{c}}\left\|v-v_{c}\right\|_{D_{j}}^{2} \leq c\left(\frac{d_{j}}{h}\right)^{2}\|v\|_{A_{j}}^{2},
$$

Combining above discussion with Theorem 6.9, we obtain

Theorem 11.9. The convergence rate of the two-level AMG based on energy minimization is bounded as following

$$
\|E\|_{A} \leq 1-\mu,
$$

with $0<\mu<1$ depends only on the size and overlaps of the subdomains $\Omega_{j}$.

\subsection{Bibliographical notes}

The energy minimization seem to encompass several algorithms: the Lagrange equations for this minimization are solved approximately in Classical AMG $(\S 12)$, while the functional is approximately minimized in the smoothed aggregation ( $\$ 13$.

For energy minimization approaches we refer to the works by (Mandel, Brezina and Vaněk 1999, Wan et al. 1999/00, Chan et al. 1998, Xu and Zikatanov 2004, Brannick and Zikatanov 2006). An interesting fact is that for regularly refined grids, given the right supports, the trace (energy) minimization prolongation recovers the coarse basis very closely, although not exactly. The small discrepancies are due effects from the boundary and the further in graph distance the coarse grid basis function is, the closer it is to piece-wise linear.

The extensive numerical experiments reported in (Wan et al. 1999/00, Wan 1998, Mandel et al. 1999, Xu and Zikatanov 2004) show that the energy minimizing basis leads to uniformly convergent two-grid and multigrid methods for many problems of practical interest and especially for problems with rough coefficients. These methods also provide framework for numerical homogenization and are related to the homogenization methods in (Grauschopf, Griebel and Regler 1997) and the $\mathbf{M}^{3}$ techniques in (Lipnikov, Moulton and Svyatskiy 2011).

The "smoothed-aggregation" approach in the algebraic multigrid method, as well as theoretical framework is reported in works by Vaněk, Mandel and Brezina (Vaněk, Mandel and Brezina 1998, Mandel et al. 1999, Vaněk, Mandel and Brezina 1996a) an explicit relation is drawn between the construction of a base for the coarse space and the "energy" of the basis functions. As pointed out in (Wan et al. 1999/00) and (Mandel et al. 1999), this can be viewed as one (or several) steps toward obtaining basis functions minimizing a quadratic (energy) functional which we consider above.

Different sparsity patterns including long distance energy minimizing prolongations are explored in (Olson, Schroder and Tuminaro 2011a), and for anisotropic problems in (Schroder 2012).

Constrained energy minimization preserving multiple vectors is considered in (Vassilevski and Zikatanov 2006). In the FE settings, when the element stiffness matrices are 
available, local energy minimization provides coarse spaces which result in a uniform two level convergence (Kolev and Vassilevski 2006).

\section{Classical AMG}

A coarse space in classical AMG is always viewed as a subspace defined via a prolongation (interpolation) matrix. Its dimension, $n_{c}$, is a fraction of the dimension of the finer space. Popular interpolation schemes in classical AMG are direct, standard, or multipass interpolation. The matrix representations of such interpolations are of the form $P=\left(\begin{array}{c}W \\ I\end{array}\right)$, with $W \in \mathbb{R}^{n_{F} \times n_{C}}$, and they can be viewed as sparse approximations to the so called "ideal" interpolation with $W=-A_{F F}^{-1} A_{F C}$, which is, in general, a full matrix. Here, the matrix $A_{F F}$ is the block of the matrix corresponding to the $F$-points and $A_{F C}$ is the block corresponding to the connections between the $C$-points and $F$-points. The splitting of vertices of the adjacency graph corresponding to $A$ in subsets $F$ and $C$ is done using one of the coarsening strategies described in $\$ 9$.

\subsection{Coarse spaces in classical $A M G$}

The coarsening algorithm in classical AMG uses a splitting of the set of vertices $\{1,2, \ldots, n\}$ of the graph corresponding to $A$ into two disjoint sets

$$
\mathcal{C} \cup \mathcal{F}=\{1,2, \ldots, n\}, \quad C \cap \mathcal{F}=\emptyset .
$$

where $C$ is a maximal independent set where the independence is with respect to the graph of the strength operator $S$ defined in $\$ 8.2$ and the splitting is referred to as a " $C / F$-splitting". A simple greedy $C / F$-splitting algorithm is introduced in $\$ 9.3 .1$. Algorithm 5 .

We assume that $V$ is equipped with a basis $\left\{\phi_{k}\right\}_{k=1}^{n}$ and we let $n_{f}=|\mathcal{F}|, n_{c}=|C|$ to denote the cardinality of the sets forming the $C / F$ splitting. Not always, but when convenient we we assume that

$$
\mathcal{F}=\left\{1, \ldots, n_{f}\right\} \text { and } C=\left\{n_{f}+1, \ldots, n\right\} .
$$

In this case, the high frequency space $V_{h f}$ as defined in (9.2) can be written as

$$
V_{h f}=\operatorname{span}\left\{\phi_{j}, j \in \mathcal{F}\right\} .
$$

Following the procedure in $\$ 9$, we first proceed to identify a tentative coarse space $W_{c}$. One easiest choice is as follows:

$$
W_{c}=\operatorname{span}\left\{\phi_{j}, j \in C\right\} .
$$

Obviously the above tentative space satisfies 9.3 , namely

$$
V=V_{h f} \oplus W_{c} .
$$

But $W_{c}$ is hardly a low frequency space. Given a basis function in $W_{c}, \phi_{j_{k}}$, we filter 
out its high frequency component to obtain the following coarse basis function,

$$
\phi_{k, c}:=\left(I-\Pi_{h f}\right) \phi_{j_{k}}=\phi_{j_{k}}+\sum_{j \notin C} p_{j k} \phi_{j}
$$

Here $P_{h f}$ is the $(\cdot, \cdot)_{A}$ orthogonal projection onto $V_{h f}$. By the definition of $\Pi_{h f}$ :

$$
\Pi_{h f} \phi_{k}= \begin{cases}\phi_{k}, & \text { if } k \in \mathcal{F}, \\ -\sum_{j \in \mathcal{F}} p_{j k} \phi_{j}, & \text { if } k \in \mathcal{C},\end{cases}
$$

where $p_{j k}$ satisfies

$$
-\sum_{j \in \mathcal{F}} p_{j k}\left(\phi_{j}, \phi_{i}\right)_{A}=\left(\phi_{k}, \phi_{i}\right)_{A}, \quad \text { for all } i \in \mathcal{F}, \quad k \in C .
$$

In a matrix notation, with the ordering given by (12.2), the matrix form of the equations in 12.6 is

$$
A_{F F} W=A_{F C}, \quad \text { where } \quad A=\left(\begin{array}{ll}
A_{F F} & A_{F C} \\
A_{F C}^{T} & A_{C C}
\end{array}\right) .
$$

Here, $W_{j k}=p_{j k}, j \in \mathcal{F}, k \in C$, where $p_{j k}$ are the coefficients given in 12.6.

In view of Lemma 9.3, we have $S=I-\Pi_{h f} . V_{c}$ is the span of the functions in (12.5)

$$
V_{c}=\operatorname{span}\left\{\phi_{k, c}\right\}_{k=1}^{n_{c}}=\operatorname{Range}\left(I-P_{h f}\right)
$$

We note that

$$
\left(\phi_{1, c}, \ldots, \phi_{n_{c}, c}\right)=\left(\phi_{1}, \ldots, \phi_{n}\right)\left(\begin{array}{c}
W \\
I
\end{array}\right)
$$

Thus the corresponding prolongation matrix is

$$
P=\left(\begin{array}{c}
W \\
I
\end{array}\right), \quad W=-A_{F F}^{-1} A_{F C} .
$$

The functions $\left\{\phi_{k, H}\right\}$ given in 12.5 form a basis of $V_{c}$ similar to geometric multigrid method for Lagrangian finite elements. We denote the prolongation matrix defined in $(12.8)$ by $P^{\text {opt }}$ and refer to it as the ideal interpolation below.

The following result can be easily established.

Lemma 12.1. If $A$ satisfies $A \mathbf{1}=0$, the solution of (12.7) also satisfies the constraint in (11.12), namely, $W \mathbf{1}_{n_{c}}=\mathbf{1}_{n_{F}}$.

Next, we introduce the set of the prolongations $\mathcal{P}_{C}$,

$$
\mathcal{P}_{C}=\left\{P \mid P=\left(\begin{array}{c}
W \\
I
\end{array}\right)\right\} \text {. }
$$

and recall from the definition given in 5.26 in $\$ 11$, that

$$
\mathcal{P}_{C} \subset \eta \mathcal{X},
$$


for some constant $\eta>0$. For example, $\eta \equiv \max _{1 \leq j \leq n}\left|D_{j j}\right|$ if $\bar{R}^{-1} \equiv D$, where $D$ is the diagonal of $A$. Equivalently, $P \in \mathcal{P}_{C}$ means that the coarse grid basis is defined as

$$
\phi_{k, H}=\phi_{j_{k}}+v_{f}, \quad j_{k} \in C, \quad v_{f} \in V_{f}, \quad k=1, \ldots, n_{c} .
$$

Clearly, all elements of $\mathcal{P}_{C}$ are full rank and we also have that $P^{\text {opt }} \in \mathcal{P}_{C}$ by definition.

We have the following theorem, showing that the coarse grid matrix corresponding to $V_{c}^{\mathrm{opt}}=\operatorname{Range}\left(P^{\mathrm{opt}}\right)$ has a minimal trace.

Theorem 12.2. If we fix the set of indexes $C$ and coarse grid degrees of freedom then for $P^{\text {opt }}$ we have

$$
P^{\mathrm{opt}}=\underset{P \in \mathcal{P}_{C}}{\arg \min } \operatorname{trace}\left(P^{T} A P\right) .
$$

Furthermore, if we denote $A_{c}=\left(P^{\mathrm{opt}}\right)^{T} A P^{\mathrm{opt}}$, then

$$
\left\|v_{c}\right\|_{A_{c}}^{2}=\min \left\{\|v\|_{A}^{2} \mid\left\langle\phi_{j_{k}}^{\prime}, v\right\rangle=v_{c, k}, k=1, \ldots, n_{c}\right\}
$$

Proof. The relation (12.9) follows from the following simple identities.

$$
\begin{aligned}
\operatorname{trace}\left(A_{c}\right) & =\sum_{k=1}^{n_{c}}\left\|\phi_{k, H}\right\|_{A}^{2}=\sum_{k=1}^{n_{c}}\left\|\left(I-P_{h f}\right) \phi_{j_{k}}\right\|_{A}^{2} \\
& =\sum_{k=1}^{n_{c}} \min _{v_{k} \in V_{h f}}\left\|\phi_{j_{k}}+v_{k}\right\|_{A}^{2} \\
& =\min \left\{\sum_{k=1}^{n_{c}}\left\|\phi_{j_{k}}+v_{k}\right\|_{A}^{2} \mid v_{k} \in V_{h f}, k=1, \ldots, n_{c}\right\} \\
& =\min _{P \in \mathcal{P}_{C}} \operatorname{trace}\left(P^{T} A P\right) .
\end{aligned}
$$

To prove (12.10), we note that any $v \in V$ such that $\left\langle\phi_{j_{k}}^{\prime}, v\right\rangle=v_{c, k}$ can be written as

$$
v=w_{c}+v_{f}, \text { where } w_{c}=\sum_{k=1}^{n_{c}} v_{c, k} \phi_{j_{k}} .
$$

By the definition of $P_{h f}$, we have $\left(I-P_{h f}\right) v_{f}=0$ and $v_{c}=\left(I-P_{h f}\right)\left(v-v_{f}\right)=$ $\left(I-P_{h f}\right) v$. We then have

$$
\|v\|_{A}^{2}=\left\|P_{h f} v\right\|_{A}^{2}+\left\|\left(I-P_{h f}\right) v\right\|_{A}^{2}=\left\|P_{h f} v\right\|_{A}^{2}+\left\|v_{c}\right\|_{A_{c}}^{2} \geq\left\|v_{c}\right\|_{A_{c}}^{2}
$$

Theorem 12.2 shows that the minimizer $P$ satisfies the equation 12.7). By Theorem 12.2 it follows that the minimizer of 11.12 and the solution to 12.7) are the same in case $A 1=0$. 


\subsection{Quasi-optimality of ideal interpolation}

\subsection{Quasi-optimality of ideal interpolation}

The following two-level convergence result is well known (see, for example (MacLachlan, Manteuffel and McCormick 2006)).

Theorem 12.3. For $P^{\text {opt }}$ defined as solution to the minimization problem (11.12), or, equivalently the solution to $(12.7)$, the two-level AMG method with prolongation $P^{\text {opt }}$ converges with a rate $\|E\|_{A} \leq 1-\delta$, with $\delta$ a constant depending only on the maximum degree of the vertices in the graph corresponding to $A$ and the threshold $\theta$ in choosing the strong connections.

Proof. According to Corollary 5.20, we only need to verify that $V_{h f}$ consists of $\delta$-algebraic high frequencies as defined in Definition 5.16. Clearly, from the discussion in $\$ 7.2$, we can assume $A$ is an $M$-matrix with all connections being strong connections. We consider the graph corresponding to $A$ by and recall that the set of coarse grid degrees of freedom is a maximal independent set of vertices in this graph. By the definition of the strength of connection, for any $j \in\{1, \ldots, n\}$ and any $i \in N(j)$ we have

$$
a_{j j}=\sum_{k \in N_{j}}-a_{j k} \leq\left|N_{j}\right| \max _{k \in N_{j}}\left(-a_{j k}\right) \leq-\frac{\left|N_{j}\right|}{\theta} a_{j i}
$$

Next, for any $j \in F$, let $k_{j} \in C$ be such that such that $\left|a_{k_{j}, j}\right|>0$. Such $k_{j}$ exists because the set of $C$-vertices is a maximal independent set. This choice is not unique, and we just fix one such index $k_{j}$ for every $j$. Using the notation from $\$ 9$, and the fact that $v \in V_{h f}$ vanishes at the $C$-vertices, we obtain

$$
\begin{aligned}
\|v\|_{\bar{R}^{-1}}^{2} & \leq c^{D}\|v\|_{D}^{2}=c^{D} \sum_{j \in F} a_{j j} v_{j}^{2}=c^{D} \sum_{j \in F} a_{j j}\left(v_{j}-v_{k_{j}}\right)^{2} \\
& \leq c^{D} \sum_{j \in F}-\frac{\left|N_{j}\right|}{\theta} a_{j, k_{j}}\left(v_{j}-v_{k_{j}}\right)^{2} \\
& \leq \max _{j}\left(\left|N_{j}\right|\right) \frac{c^{D}}{\theta} \sum_{(i, j) \in \mathcal{E}}-a_{i j}\left(v_{i}-v_{j}\right)^{2} \\
& \leq \max _{j}\left(\left|N_{j}\right|\right) \frac{c^{D}}{\theta}\|v\|_{A}^{2} .
\end{aligned}
$$

We note that interpolations like $P^{\text {opt }}$ are not (with possible exception of 1D problems) used in practice, because the prolongation matrix $P^{\text {opt }}$ could have a lot of fill-in and $\left(P^{\text {opt }}\right)^{T} A P^{\text {opt }}$ is dense. It is, however, also important to note that sparse approximations to $P^{\mathrm{opt}}$ are what is used in practice. Thus, the energy minimization technique in constructing coarse space may be viewed as a motivation for the other AMG techniques used to construct approximations of $V_{c}^{\mathrm{opt}}$. For example, most of the known techniques approximate the minimizer of the functional 
$\mathcal{F}$ given in $\$ 11.2$, Equation (11.12): (1) The prolongation matrices constructed in the classical AMG framework approximate the solution to equation (12.7) over the subset of $\mathcal{P}_{C}$ consisting of sparse matrices; (2) The energy minimization techniques outlined in $\$ 11.2$, and the smoothed aggregation considered in $\$ 13.3$ minimize (approximately) the trace of the coarse grid matrix $\mathcal{F}(P)$ over a set of sparse matrices $P$.

\subsection{Construction of prolongation matrix $P$}

\subsubsection{Prolongation}

An intuitive idea to find an approximate solution of the problem 12.7) is to use some basic iterative methods such as Jacobi method and then properly rescale the coefficients of $W$ so that it satisfies

$$
W \mathbf{1}_{n_{c}}=\mathbf{1}_{n_{f}},
$$

and also fits into a sparsity pattern:

$$
W \in \mathbb{R}_{S}^{n_{f} \times n_{c}} .
$$

Following this idea, we construct the interpolations used in classical AMG (Trottenberg et al. 2001, Section A.7): (1)direct interpolation; (2) standard interpolation; and (3) multi-pass interpolation in a unified fashion.

- Direct interpolation: Direct interpolation approximates the solution to (12.7)(12.12) by one Jacobi iteration with initial guess $W_{0}=0$, namely,

$$
W_{1}=-D_{F F}^{-1} A_{F C} \text {. }
$$

In order to satisfy the constraint 12.11 , we rescale $W_{1}$, to obtain that

$$
W=M\left(-D_{F F}^{-1} A_{F C}\right)=\left[\operatorname{diag}\left(A_{F C} \mathbf{1}\right)\right]^{-1} A_{F C} .
$$

where $M$ is a rescaling operator defined as follows

$$
M(Y)=[\operatorname{diag}(Y \mathbf{1})]^{-1} Y
$$

- Standard interpolation: The construction of prolongation matrix via standard interpolation can be viewed in several different ways. The most natural way probably is to view it as a smoothing of the direct interpolation again followed by rescaling. Indeed, assuming that the smooth error $\left(e_{F}^{T}, e_{C}^{T}\right)$ satisfies

$$
A_{F F} e_{F}+A_{F C} e_{C}=0,
$$

we approximate this equation (using the same notation $e_{F}, e_{C}$ for the approximations) by (see (Trottenberg et al. 2001)):

$$
\begin{aligned}
e_{F} & =-D_{F F}^{-1} A_{F C} e_{C}+D_{F}^{-1} A_{F F}\left(D_{F F}^{-1} A_{F C}\right) e_{C} \\
W & =\left(I-D_{F F}^{-1} A_{F F}\right) W^{1}, \quad W^{1}=-D_{F F}^{-1} A_{F C} .
\end{aligned}
$$




\subsection{Construction of prolongation matrix $P$}

This is equivalent to a Jacobi smoothing iteration applied to the unscaled direct interpolation $W^{1}$. Rescaling is also needed for the standard interpolation and the final formula is

$$
W=\left[\operatorname{diag}\left(\left(I-D_{F F}^{-1} A_{F F}\right) W^{1} \mathbf{1}\right)\right]^{-1}\left(I-D_{F F}^{-1} A_{F F}\right) W^{1} .
$$

The similarity with the smoothed aggregation discussed in $\$ 13.3$ is obvious, as this is indeed a smoothing applied to $W^{1}$.

- Multipass interpolation: The multipass interpolation is an approximation to the solution of (12.7) when the $C / F$ splitting has been constructed by means of aggressive coarsening, namely, using the $(m, l)$-strong connection defined in Definition 9.7. The set of $F$ is divided into $l$ disjoint subsets $F_{1}, F_{2}, \ldots, F_{l}$ as follows: we first define the distance from a point $j$ to a subset of points $C$,

$$
\operatorname{dist}(j, C)=\min \{\operatorname{dist}(j, i): i \in C\} .
$$

Then, we set

$$
F_{k}=\{j \in \mathcal{F}: \operatorname{dist}(j, C)=k\}, \quad k=1,2, \ldots, l, \quad l=\max \{\operatorname{dist}(j, C): j \in F\} .
$$

Then $A_{F F}, A_{F C}$ can be written as the following block matrices:

$$
A_{F F}=\left(\begin{array}{cccc}
A_{F_{1} F_{1}} & A_{F_{1} F_{2}} & \cdots & A_{F_{1} F_{l}} \\
A_{F_{2} F_{1}} & A_{F_{2} F_{2}} & \cdots & A_{F_{2} F_{l}} \\
\vdots & \vdots & \ddots & \vdots \\
A_{F_{l} F_{1}} & A_{F_{l} F_{2}} & \cdots & A_{F_{l} F_{l}}
\end{array}\right), \quad A_{F C}=\left(\begin{array}{c}
A_{F_{1} C} \\
A_{F_{2} C} \\
\vdots \\
A_{F_{l} C}
\end{array}\right) .
$$

We can also write $W$ block-wise as $W^{T}=\left(W_{F_{1}}^{T}, W_{F_{2}}^{T}, \ldots, W_{F_{l}}^{T}\right)$ and then (12.7) takes the form

$$
\left\{\begin{array}{c}
A_{F_{1} F_{1}} W_{F_{1}}+A_{F_{1} F_{2}} W_{F_{2}}+\cdots+A_{F_{1} F_{l}} W_{F_{l}}+A_{F_{1} C}=0 \\
A_{F_{2} F_{1}} W_{F_{1}}+A_{F_{2} F_{2}} W_{F_{2}}+\cdots+A_{F_{2} F_{l}} W_{F_{l}}+A_{F_{2} C}=0 \\
\vdots \\
A_{F_{l} F_{1}} W_{F_{1}}+A_{F_{l} F_{2}} W_{F_{2}}+\cdots+A_{F_{l} F_{l}} W_{F_{l}}+A_{F_{l} C}=0
\end{array}\right.
$$

To define the entries of $W_{F_{k}}$ via multipass interpolation we use the following steps:

1 For $k=1$, use direct interpolation to approximate $W_{F_{1}}$. More precisely, we write the first equation in $(12.18)$ as

$$
A_{F_{1} F_{1}} W_{F_{1}}+\hat{A}_{F_{1} C}=0
$$

with $\hat{A}_{F_{1} C}=A_{F_{1} F_{2}} W_{F_{2}}+\cdots+A_{F_{1} F_{l}} W_{F_{l}}+A_{F_{1} C}$. Then apply direct interpolation and write $W_{F_{1}}$ as a function of $W_{F_{j}}, j>1$.

2 While $k<l$ :

(a) Write the $(k+1)$-st equation in (12.18) substituting the expressions for $W_{F_{m}}, m<(k+1)$ obtained from the previous steps. The $(k+1)$-th 
equations then has the form

$$
\hat{A}_{F_{k+1} F_{k+1}} W_{F_{k+1}}+\hat{A}_{F_{k+1} F_{k+2}} W_{F_{k+2}}+\cdots \hat{A}_{F_{k+1} F_{l}} W_{F_{l}}+\hat{A}_{F_{k+1} C}=0 .
$$

(b) Apply direct interpolation to (12.19) to write $W_{F_{k+1}}$ as a function of $W_{F_{j}}, j>(k+1)$.

(c) Set $k \leftarrow k+1$.

From the derivation and the definitions above we have the following set inclusions describing the sparsity patterns of the prolongations defined earlier:

$$
\begin{aligned}
& \mathrm{S}(P) \subset \mathrm{S}\left(\begin{array}{c}
A_{F C} \\
I
\end{array}\right) \quad(\text { direct interpolation 12.3.1) }) \\
& \mathrm{S}(P)=\mathrm{S}\left(\begin{array}{c}
\widehat{A}_{F C} \\
I
\end{array}\right) \quad \text { (standard interpolation 12.16) } \\
& \mathrm{S}(P)=\mathrm{S}\left(\begin{array}{c}
\widehat{A}_{F_{1} C} \\
\widehat{A}_{F_{2} C} \\
\vdots \\
\widehat{A}_{F_{l} C} \\
I
\end{array}\right) \quad \text { (multipass interpolation). }
\end{aligned}
$$

\subsubsection{Interpolation preserving a given vector}

From the previous discussions, it is important to have the prolongation $P$ preserves some vectors which can represent algebraic smooth errors. The direct and standard interpolation use a diagonal scaling to make sure the prolongation preserves constant vectors, which is the kernel of scalar elliptic operators. To generalize this idea, here we introduce $\alpha \mathrm{AMG}$ interpolation which constructs prolongation matrix by choosing an initial guess that preserves the near null component $v^{(1)}$, which may not necessarily be constant vectors.

We construct $P \in \mathcal{P}_{C}$ such that it "preserves" a given vector $v$, namely,

$$
v_{F}=W v_{C} .
$$

To do this, we first pick an initial guess $W^{0}$ for $W$ (or $P$ ),

$$
W^{0}=D_{v}^{-1} A_{F C}
$$

where $D_{v}$ is a diagonal matrix such that the following identity holds

$$
v_{F}=D_{v}^{-1} A_{F C} v_{C} \text {. }
$$

It is easy to derive that the explicit formula for diagonal entries of $D_{v}$ is

$$
d_{k k}=\frac{\sum_{j \in C} a_{k j} v_{j}^{(1)}}{v_{k}^{(1)}} \text {. }
$$




\subsection{Classical AMG within the abstract AMG framework}

Then the $W$ in such "vector preserving" interpolation is obtained by applying one Jacobi iteration for the linear problem (12.7) with initial guess $W^{0}$

$$
W=D^{-1} A_{F C}+D_{F F}^{-1}\left(-A_{F C}-A_{F F} D^{-1} A_{F C}\right) .
$$

A fully detailed description of the construction a prototype vector $v$ and coarse space interpolating this vector exactly, using $\alpha$ AMG (the classical AMG version of adaptive AMG) is found in (Brezina, Falgout, MacLachlan, Manteuffel, McCormick and Ruge 2006b). The ideas, however are outlined earlier in (Brandt et al. 1982b).

\subsection{Classical $A M G$ within the abstract $A M G$ framework}

The classical AMG falls in the abstract theory we developed earlier in $\$ 5$ and $\$ 6$. To do this, we first consider an $M$-matrix relative of $A$ using the adjacency graph corresponding to a strength function. We then use an MIS algorithm to identify $C$, the set of coarse points, to form a $C / F$-splitting. We further split the set of indices $\Omega=\{1,2, \ldots, n\}$ into subsets $\Omega_{1}, \Omega_{2}, \ldots, \Omega_{J}$ so that

$$
\Omega=\bigcup_{j=1}^{J} \Omega_{j} .
$$

Then for each $j \in C$ we define

$$
\Omega_{j}:=\{j\} \bigcup F_{j}^{s}, \quad j=1, \ldots, J .
$$

where $F_{j}^{s}:=\mathcal{F} \cap s_{j}$, and $s_{j}$ is the set of strong neighbors of $j$. This depends on the definition of strength of connection. For example, in the direct interpolation we introduced in the previous section, we simply use the strength connection defined in (8.8); in the standard interpolation, we use $(m, l)$-strong connection defined in Definition 9.7 with $m=1$ and $l=2$.

For each $\Omega_{j}$ we denote

$$
\Omega_{j}=\left\{m_{1}, m_{2}, \ldots, m_{n_{j}}\right\},
$$

and let $n_{j}:=\left|\Omega_{j}\right|$, namely, $n_{j}$ is the cardinality of $\Omega_{j}$. In accordance with the notation in $\$ 6$. We then define

$$
V_{j}:=\mathbb{R}^{n_{j}},
$$

and the associated operator $\Pi_{j}: V_{j} \mapsto V$

$$
\left(\Pi_{j} v\right)_{i}=\left\{\begin{array}{ll}
p_{m_{k}, k} v_{k}, & \text { if } i=m_{k}, \\
0, & \text { if } i \notin \Omega_{j}
\end{array},\right.
$$

where $p_{m_{k}, k}$ are given weights. As all the constructions below will be based on the $M$-matrix relative of $A$, and without loss of generality, we may just use $A$ to denote the $M$-matrix relative. 
Following $\$ 6$, we introduce the operator $\chi_{j}: V \mapsto V_{j}$ :

$$
\left(\chi_{j} v\right)_{i}:=v_{m_{i}} .
$$

which takes as argument a vector $v$ and returns only the portion of it with indices are in $\Omega_{j}$. Namely, $\chi_{j} v$ is a vector in $\mathbb{R}^{n_{j}}$. It is immediate to verify that

$$
\sum_{j=1}^{J} \Pi_{j} \chi_{j}=I
$$

To estimate the convergence rate using the theory in $\$ 5$ We need to verify all the items in Assumptions 6.9. To do this, we choose a decomposition $v=\sum_{j=1}^{J} \Pi_{j} v_{j}$ with $v_{j}=\chi_{j} v$. We further define $C_{p, 2}$ as a constant depending on the overlaps in the partition $\left\{\Omega_{j}\right\}_{j=1}^{J}$,

$$
C_{o}=\max _{1 \leq j \leq J}\left|\left\{l: \Omega_{l} \cap \Omega_{j} \neq \emptyset\right\}\right| .
$$

The local operators $A_{j}$ on $V_{j}$ are defined as follows

$$
\left(A_{j} u, v\right)=\sum_{\substack{e \in \mathcal{E} \\ e \subset \Omega_{j}}} \omega_{e} \delta_{j, e} u \delta_{j, e} v .
$$

Here, $e \subset \Omega_{j}$ means the two vertices connected by $e$ are in $\Omega_{j}$, and $\delta_{j, e} u=u_{m_{k}-m_{l}}$ for $e=(k, l)$ and $\omega_{e}$ are the eights determined by the off-diagonal elements in the $M$-matrix relative of $A$. Notice that $A_{j}$ is a symmetric positive semi-definite matrix because all the weights $\omega_{e}$ are non-negative. Then 6.11) easily verified:

$$
\begin{aligned}
\sum_{j=1}^{m_{c}}\left\|\chi_{j} v\right\|_{A_{j}}^{2} & =\sum_{j=1}^{m_{c}} \sum_{\substack{e \in \mathcal{E} \\
e \subset \Omega_{j}}} \omega_{e}\left(\delta_{e} v\right)^{2} \leq C_{o} \sum_{e \in \mathcal{E}} \omega_{e}\left(\delta_{e} v\right)^{2} \\
& =C_{o}\|v\|_{A_{M}}^{2} \leq C_{o} C_{M}\|v\|_{A}^{2},
\end{aligned}
$$

If $D$ is the diagonal of $A$, then we set $D_{j}, j=1: J$ to be the restriction of $D$ on $\Omega_{j}$, namely, in $\mathbb{R}^{n_{j} \times n_{j}}$ and

$$
\left(D_{j}\right)_{i i}=D_{m_{i}, m_{i}}, \quad \text { or equivalently } \quad D_{j}=\chi_{j} D_{j} \chi_{j}^{\prime}
$$

We have the following lemma which shows 6.10.

Lemma 12.4. For $D_{j}$ defined in (12.30), the following inequality holds

$$
\left\|\sum_{j=1}^{m_{c}} \Pi_{j} w_{j}\right\|_{D}^{2} \leq C_{o} \sum_{j=1}^{m_{c}}\left\|w_{j}\right\|_{D_{j}}^{2}, \quad \forall w_{j} \in V_{j} .
$$

Proof. Recall from the definition of $\Pi_{j}$, we have

$$
\left\|\Pi_{j} v\right\|_{D} \leq\|v\|_{D_{j}}, \quad \forall v \in V_{j} .
$$


Therefore,

$$
\begin{aligned}
\left\|\sum_{j=1}^{J} \Pi_{j} v_{j}\right\|_{D}^{2} & =\left(D \sum_{i=1}^{J} \Pi_{i} v_{i}, \sum_{j=1}^{J} \Pi_{j} v_{j}\right)=\sum_{i=1}^{J} \sum_{j=1}^{J}\left(D \Pi_{i} v_{i}, \Pi_{j} v_{j}\right) \\
& =\sum_{\substack{1 \leq i, j \leq J \\
\Omega_{i} \cap \Omega_{j} \neq \emptyset}}\left(D \Pi_{i} v_{i}, \Pi_{j} v_{j}\right) \leq \sum_{\substack{1 \leq i, j \leq J \\
\Omega_{i} \cap \Omega_{j} \neq \emptyset}} \frac{\left\|\Pi_{j} v_{i}\right\|_{D}^{2}+\left\|\Pi_{j} v_{j}\right\|_{D}^{2}}{2} \\
& \leq C_{o} \sum_{j=1}^{J}\left\|v_{j}\right\|_{D_{j}}^{2} .
\end{aligned}
$$

We choose the local coarse spaces $V_{j}^{c}$ as

$$
V_{j}^{c}:=\operatorname{span}\left\{\mathbf{1}_{n_{j}}\right\}
$$

Then by definition, we have

$$
\mu_{j}\left(V_{j}^{c}\right)=\frac{1}{\lambda_{j}^{(2)}},
$$

where $\lambda_{j}^{(2)}$ is the second smallest eigenvalue of the matrix $D_{j}^{-1} A_{j}$. The global coarse space $V_{c}$ is then obtained by [6.13), and is

$$
V_{c}=\operatorname{span}\left\{P_{1}, P_{2}, \cdots, P_{J}\right\} .
$$

Finally, by Theorem 5.2, the converges rate of this two-level geometric multigrid method depends on the $\min _{j}\left(\lambda_{j}^{(2)}\right)$. If the discrete Poincaré inequality is true for each $V_{j}$, namely,

$$
\inf _{v_{c} \in V_{j}^{c}}\left\|v-v_{c}\right\|_{D_{j}}^{2} \leq c_{j}\|v\|_{A_{j}}^{2}, \quad \forall v \in V_{j},
$$

with $c_{j}$ to be a constant, then the two-level classical AMG method converges uniformly.

\subsection{Bibliographical notes}

The classical coarse space definition in AMG was introduced in (Brandt et al. 1982b), and then somewhat improved in (Stüben 1983, Brandt et al. 1985, Ruge and Stüben 1987).

Despite its great success in practical applications, classical AMG algorithms still lack solid theoretical justifications beyond theory for two-level methods. It is important to note that a multigrid method that converges uniformly in the two-level case with an exact coarse grid solver may not converge uniformly in the multilevel case (see Brandt (Brandt 1986) and Ruge and Stüben (Ruge and Stüben 1987)). For classical AMG, the early theoretical studies of convergence date back to the 1980's 
(see (Mandel 1988, McCormick 1982, Brandt 1986, Ruge and Stüben 1987)). A survey of these results by Stüben is found in the monograph (Trottenberg et al. 2001) where also the three classical prolongation constructions (direct, standard and multipass) are given. In all cases, it is crucial to define coarsening and interpolation operators so that the interpolation error is uniformly bounded. The role of the ideal interpolation as minimizer of an upper bound for the convergence rate was emphasized in (Falgout and Vassilevski 2004).

\section{Aggregation-based AMG}

Aggregation (or agglomeration) refers to an algorithm that splits the set of vertices of the graph of the filtered matrix as a union of non-overlapping subsets (aggregates) (each of which forms a connected sub-graph):

$$
\{1, \ldots, n\}=\bigcup_{j=1}^{J} \mathcal{A}_{j}, \quad \mathcal{A}_{i} \bigcap \mathcal{A}_{j}=\emptyset, i \neq j,
$$

Such a partition can be obtained algorithms described in $\$ 9$

If we are solving a finite element system, the partition (13.1) would correspond to a non-overlapping decomposition of $\Omega$

$$
\Omega=\bigcup_{j=1}^{J} \Omega_{j}, \quad \Omega_{i} \bigcap \Omega_{j}=\emptyset, i \neq j
$$

such that $\mathcal{A}_{j}$ contains the indices associated with the enumerations of the vertices in the subdomain $\Omega_{j}$.

We denote the elements in $\mathcal{A}_{j}$ by

$$
\mathcal{A}_{j}=\left\{m_{1}, m_{2}, \ldots, m_{n_{j}}\right\},
$$

and let $n_{j}:=\left|\mathcal{A}_{j}\right|$, namely, $n_{j}$ is the number of elements in $\mathcal{A}_{j}$.

\subsection{Unsmoothed aggregation: preserving 1 kernel vector}

Using the framework we introduced in $\$ 6$, we define

$$
V_{j}:=\mathbb{R}^{n_{j}},
$$

and the associated operator $\Pi_{j}: \mathbb{R}^{n_{j}} \mapsto \mathbb{R}^{n}$ is the trivial extension of $v \in \mathbb{R}^{n_{j}}$

$$
\begin{gathered}
\left(\Pi_{j} v\right)= \begin{cases}v_{k} & i=m_{k}, \\
0 & i \notin \mathcal{A}_{j} .\end{cases} \\
P=\left(p_{1}, p_{2}, \ldots, p_{J}\right), \quad p_{j}=\Pi_{j} \mathbf{1}_{n_{j}} .
\end{gathered}
$$


This prolongation matrix obviously satisfies

$$
P \mathbf{1}_{J}=\sum_{j-1}^{J} P_{j}=\mathbf{1}_{n} .
$$

The local coarse space $V_{j}^{c}$ is

$$
V_{j}^{c}:=\operatorname{span}\left\{\mathbf{1}_{n_{j}}\right\}
$$

Then the global coarse space $V_{c}$ is obtained by (6.13). In fact

$$
V_{c}=\operatorname{span}\left\{p_{1}, p_{2}, \ldots, p_{J}\right\} .
$$

We note that

$$
\left(\phi_{1, c}, \ldots, \phi_{J, c}\right)=\left(\phi_{1}, \ldots, \phi_{n}\right) P
$$

where

$$
\phi_{j, c}=\sum_{k \in \mathcal{A}_{j}} \phi_{k}, \quad j=1,2, \ldots, J
$$

Furthermore, $\Pi_{j}$ corresponds to matrix representation of the operator $I_{h}\left(\phi_{j}^{H} \cdot\right)$ with the coarse grid basis $\phi_{j, c}$ defined above. In view of $\$ 10.3$ (see especially (10.14)). Aggregation based AMG can be also viewed as a GMG method.

The above procedure gives a full description of the un-smoothed aggregation AMG method. We can use the framework in $\$ 6$ to carry out a two-level convergence analysis. The local matrices $A_{j}$ are defined exactly the same as in the case of classical AMG in $\$ 12.4$. namely, we write $A$ as in (12.4) and then define $A_{j}$ by (12.28). The matrices $D_{j}$ are defined as the restriction of the diagonal of $A$ on $\Omega_{j}$ as in (12.30). Using the same argument as in $\$ 12.4$. Assumption 6.9 is verified by 12.29 and Lemma 12.4 . Theorem 5.2 can then be applied to prove that the two-level unsmoothed aggregation method has a convergence rate depending only on the local Poincaré constants in (12.36.

\subsection{Unsmoothed aggregation: preserving multiple vectors}

One great advantage of aggregation AMG method is that it can be easily extended to case that the stiffness matrix $A$ has multiple kernel or near-kernel vectors.

To give an illustration how to construct prolongation preserving more than one vector, we consider the $2 \mathrm{D}$ elasticity problems. In this case, we have 3 functions, namely the rigid body motion, to preserve:

$$
u_{1}=\left(\begin{array}{l}
1 \\
0
\end{array}\right), u_{2}=\left(\begin{array}{l}
0 \\
1
\end{array}\right), u_{3}=\left(\begin{array}{c}
-y \\
x
\end{array}\right)
$$


The corresponding vectors are

$$
\zeta_{1}=\left(\begin{array}{c}
1 \\
0 \\
1 \\
0 \\
\vdots \\
1 \\
0
\end{array}\right), \zeta_{2}=\left(\begin{array}{l}
0 \\
1 \\
0 \\
1 \\
\vdots \\
0 \\
1
\end{array}\right) \text { and } \zeta_{3}=\left(\begin{array}{c}
-y_{1} \\
x_{1} \\
-y_{2} \\
x_{2} \\
\vdots \\
-y_{n} \\
x_{n}
\end{array}\right) \in \mathbb{R}^{2 n}
$$

On each aggregate $\mathcal{A}_{j}$, we consider

$$
\zeta_{1}^{(j)}=\left(\begin{array}{c}
1 \\
0 \\
1 \\
0 \\
\vdots \\
1 \\
0
\end{array}\right), \zeta_{2}^{(j)}=\left(\begin{array}{c}
0 \\
1 \\
0 \\
1 \\
\vdots \\
0 \\
1
\end{array}\right) \text { and } \zeta_{3}^{(j)}=\left(\begin{array}{c}
-y_{m_{1}} \\
x_{m_{1}} \\
-y_{m_{2}} \\
x_{m_{2}} \\
\vdots \\
-y_{m_{n_{j}}} \\
x_{m_{n_{j}}}
\end{array}\right) \in \mathbb{R}^{2 n_{j}}
$$

The prolongation matrix is then given by

$$
P=\left(p_{1}, p_{2}, \ldots, p_{J}\right) \in \mathbb{R}^{(2 n) \times(3 J)} \quad P_{j}=\Pi_{j}\left(\zeta_{1}^{(j)}, \zeta_{2}^{(j)}, \zeta_{3}^{(j)}\right) \in \mathbb{R}^{(2 n) \times 3} .
$$

This prolongation matrix satisfies

$$
P\left(\mathbf{1}_{J} \otimes e_{j}\right)=\zeta_{j}, 1 \leq j \leq 3 .
$$

The rest of the AMG algorithm based on this prolongation matrix is similar to the 1-vector case.

Extension from 3-vector case as mentioned above to arbitrary $m$-vector is straightforward. We denote $m=\operatorname{dim}(N(A))$ and consider the case when $m \geq 1$. We now assume that we are given $m$ vectors $\left\{\zeta_{j}\right\}_{j=1}^{m}$ which are linearly independent and $m \ll n$ and we can then proceed to construct a prolongation $P$ such that $P\left(\mathbf{1}_{n_{c}} \otimes e_{j}\right)=\zeta_{j}$, $j=1: m$.

We would like to point out for the multiple kernel or near-kernel vector case, we often need some geometric information to describe the corresponding vectors. In the finite element case, these vectors should be obtained by taking the canonical interpolation of the corresponding kernel functions of the underlying partial differential operators and we then split these vectors into different aggregates by using the local degrees of freedom associated with different aggregates.

\subsection{Smoothed aggregation}

The aggregation procedure described above provides a simple and yet efficient AMG method. The resulting method is known as un-smoothed aggregation AMG 


\subsection{Smoothed aggregation}

(UA-AMG) method. Such a terminology may be justified by examining the shape of the basis function as defined by (13.8). A typical basis in this form, is not smooth. There is a procedure to smooth out these basis function by using a smoother to smooth these basis functions, which is equivalent to a few applications of smoothing on the prolongation matrix as defined (13.5) as follows:

$$
P_{S}=\left(I-R_{S} A\right)^{v} P, \quad \text { for some } v \geq 1 .
$$

A typical choice is the scaled Jacobi smoother with $R_{S}=\omega D^{-1}$. We note that, in view of 13.9, if $\zeta_{j}$ is in the kernel of $A$, we still have

$$
P_{S}\left(\mathbf{1}_{J} \otimes e_{j}\right)=\zeta_{j}, 1 \leq j \leq m .
$$

Conceivably, a bigger $v$ in 13.10 would lead to an AMG algorithm that has a better convergence rate. But a bigger $v$ in $(13.10)$ also means a denser $P_{S}$ and hence a denser $A_{c}$, and hence a more expensive setup for the resulting AMG algorithm. As an example, the graphs of the coarse grid matrices corresponding to unsmoothed and smoothed aggregation are shown in Figure 13.13. Clearly, the smoothed aggregation graph is denser than the unsmoothed one.
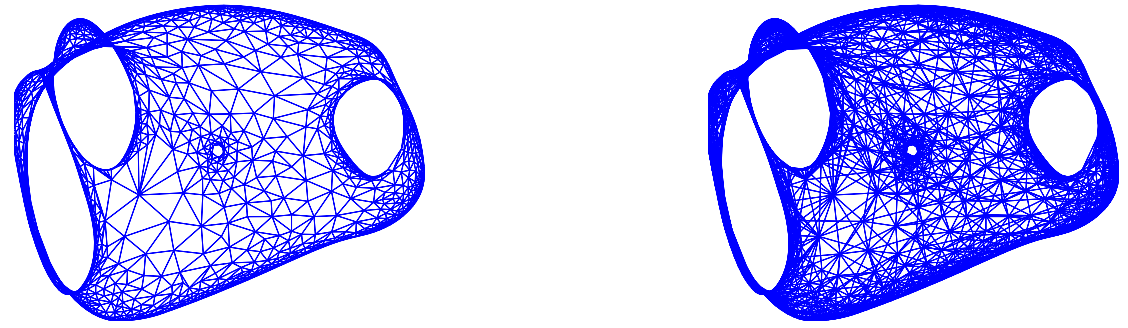

Figure 13.13. Graph of the coarse grid matrix corresponding to the unsmoothed aggregation (left) and the "denser" graph for the coarse grid matrix obtained by smoothed aggregation (right).

In view of Lemma 9.3, the basis function (13.8) and the resulting UA-AMG corresponds to the use of tentative coarse space $W_{c}$. The smoothed basis functions by means of (13.10) and resulting SAMG corresponds to the use of the $V_{s}$ in Lemma 9.3 with $S=\left(I-R_{S} A\right)^{v}$. 


\subsection{Bibliographical notes}

One of the first aggregation algorithms comes from applications in economics and is due Vakhutinsky in 1979 (Vakhutinsky et al. 1979). Later, aggregation methods have been developed and used for solving discrete elliptic problems by Blaheta (Blaheta 1986) and in the calculation of stationary points of Markov chains by Marek (Marek 1991).

A special class of aggregation coarsening method is based on matching in graphs (also known as pairwise aggregation) and such methods were employed in (Kim et al. 2003) and for nonsymmetric problems in (Kim, Xu and Zikatanov 2004). Aggregations using matching in graphs were further used in (Notay 2010, Brannick, Chen and Zikatanov 2012, Livne and Brandt 2012, D'Ambra and Vassilevski 2014, D'Ambra and Vassilevski 2013). To improve the scalability of the unsmoothed aggregation a number of algorithms were developed: in (Kim et al. 2003) variable $V$-cycle was used; (Notay and Vassilevski 2008a) propose non-linear Krylov subspace acceleration; in (Olson, Schroder and Tuminaro 2011b) a procedure for correcting the energy of the coarse-level Galerkin operator is designed.

The aggregation, especially the unsmoothed aggregation, can be used in conjunction with nonlinear (variable-step/flexible) preconditioning methods to result in an optimal algorithm. Such nonlinear methods are called Algebraic Multilevel Iteration Methods and were introduced in (Axelsson and Vassilevski 1991). Nonlinear multilevel preconditioners were proposed and an additive version of them was first analyzed in (Axelsson and Vassilevski 1994) (see also (Golub and Ye 1999/00, Notay 2000, Saad 2003b, Kraus 2002). In these nonlinear multilevel preconditioners, $n$ steps of a preconditioned CG iterative method provide a polynomial approximation of the inverse of the coarse grid matrix. The same idea can be used to define the MG cycles, as shown in (Vassilevski 2008b, Notay and Vassilevski 2008b). A comprehensive convergence analysis of nonlinear AMLIcycle multigrid method for symmetric positive definite problems has been conducted in (Hu, Vassilevski and Xu 2013). Based on classical assumptions for approximation and smoothing properties, the nonlinear AMLI-cycle MG method is shown to be uniformly convergent.

The smoothed aggregation AMG method, first developed by Míka and Vaněk (Míka and Vaněk 1992b, Míka and Vaněk 1992a) and later extended by Vaněk, Mandel, and Brezina (Vaněk et al. 1996a), was motivated by some early work on aggregation-based MG studied by R. Blaheta in (Blaheta 1986) and in his dissertation (Blaheta 1988).

A major work on the theory and SA algorithm is found in (Vaněk et al. 1998). The convergence result proved there is under the assumptions on the sparsity of the coarse grid matrix and the ratio between the number of coarse and fine degrees of freedom. For general sparse matrices, and even for general adapted finite element matrices corresponding to elliptic equations, verifying such assumptions is difficult and is yet to be done. 


\section{Problems with discontinuous and anisotropic coefficients}

A good AMG method should be robust with respect to possible heterogeneity features such discontinuous jumps and anisotropy in a given problem. These heterogeneity should be detected and properly resolved automatically in an AMG process. Extensive numerical experiments have shown that AMG such as classical AMG and SA-AMG are very robust with respect to these heterogeneities. One main technique used to detect and to resolve these heterogeneities is through the strength of connections (see $\$ 8.2$. In this section, we shall use the model problem (2.1) with two special set of coefficients, 2.9) and 2.10), to discuss how classical AMG work for problem with strong heterogeneities.

\subsection{Jump coefficients}

In this section we consider an the Classical AMG method when applied to a problem with heterogenous (jump) coefficients, namely (2.1) with 2.9]. We begin with a discussion on how the strength of connection is used to define the sparsity pattern of the prolongation.

The strength of connection measure was introduced to handle cases such as jump coefficients and anisotropies in the matrices corresponding to discretizations of scalar PDEs. An important observation regarding the classical AMG is that the prolongation matrix $P$, which defines the basis in the coarse space, uses only strong connections. We need here the strength operator $S: V \mapsto V$ defined in (8.4). We now focus on the jump coefficient problem defined in (2.9)

$$
\alpha(x)=\left\{\begin{array}{lc}
\epsilon, & x \in \Omega_{\epsilon} \\
1 & x \in \Omega_{1}
\end{array}\right.
$$

with $\epsilon$ sufficiently small so that the graph corresponding to the strength operator has at least two connected components. Directly from the definition of $S_{i}$ in 8.8 we have the following:

- The graph corresponding to the strength of connection matrix $S$ is obtained from the graph corresponding to $A$ by removing all entries $a_{i j}$ corresponding to an edge connecting a vertex from the interior of $\Omega_{\epsilon}$ to a vertex in $\bar{\Omega}_{1}$.

- In another word, in this setting we have a block-lower-triangular $S$ with at least two blocks, in which the first block corresponds to the vertices interior to $\Omega_{\epsilon}$ and the second block corresponds to the rest of the vertices.

- The considerations above apply to any configuration of the subsets $\Omega_{\epsilon}$ and $\Omega_{1}$, in fact, they can even be disconnected, form cross points and so on.

In Figures 14.14 14.16 we illustrate the strength of connection graphs and their connected components for specific coefficients $\alpha(x)$ which is defined as in (2.9) with $\epsilon=10^{-3}$. The domain $\Omega_{\epsilon}$ is a union of elements, and, $T \in \Omega_{\epsilon}$ if and only if $\left(x_{T}-\frac{1}{2}\right)\left(y_{T}-\frac{1}{2}\right)<0$, where $\left(x_{T}, y_{T}\right)$ is the barycenter of $T$. The domain $\Omega_{1}$ 
is $\Omega_{1}=\Omega \backslash \bar{\Omega}_{1}$. In Figure $14.15, \Omega_{\epsilon}$ and $\Omega_{1}$ are disconnected in the graph of the strength matrix $S$. In the graph of $S, \Omega_{\epsilon}$ is split into two connected components, we denote them by $\Omega_{\epsilon}^{(1)}$ and $\Omega_{\epsilon}^{(2)}$. $\Omega_{1}$ is split into two parts, denoted by $\Omega_{1}^{(1)}$ and $\Omega_{1}^{(2)}$, which are connected through only one point $x_{0}=\left(\frac{1}{2}, \frac{1}{2}\right)$. With a re-ordering of indices, the strength matrix for this case can be written as

$$
S=\left(\begin{array}{ccccc}
S_{\epsilon, 1} & & & & \\
& S_{\epsilon, 2} & & & \\
& & S_{11} & & \\
& & & S_{12} & \\
& & S_{x 1} & S_{x 2} & S_{x}
\end{array}\right),
$$

where $S_{\epsilon, 1}$ and $S_{\epsilon, 2}$ are two diagonal blocks corresponds to the two connected component $\Omega_{\epsilon}^{(1)}$ and $\Omega_{\epsilon}^{(2)} ; S_{11}$ and $S_{12}$ are two diagonal blocks corresponds to the two connected component $\Omega_{1}^{(1)}$ and $\Omega_{1}^{(2)} ; S_{0}$ is the diagonal entry corresponds to the grid point $x_{0} ; S_{x 1}$ and $S_{x 2}$ are low rank matrices with only a few nonzero entries ( 2 nonzero entries in this example) which contain the connections from $x_{0}$ to points in $\Omega_{1}^{(1)}$ and $\Omega_{1}^{(2)}$ respectively.
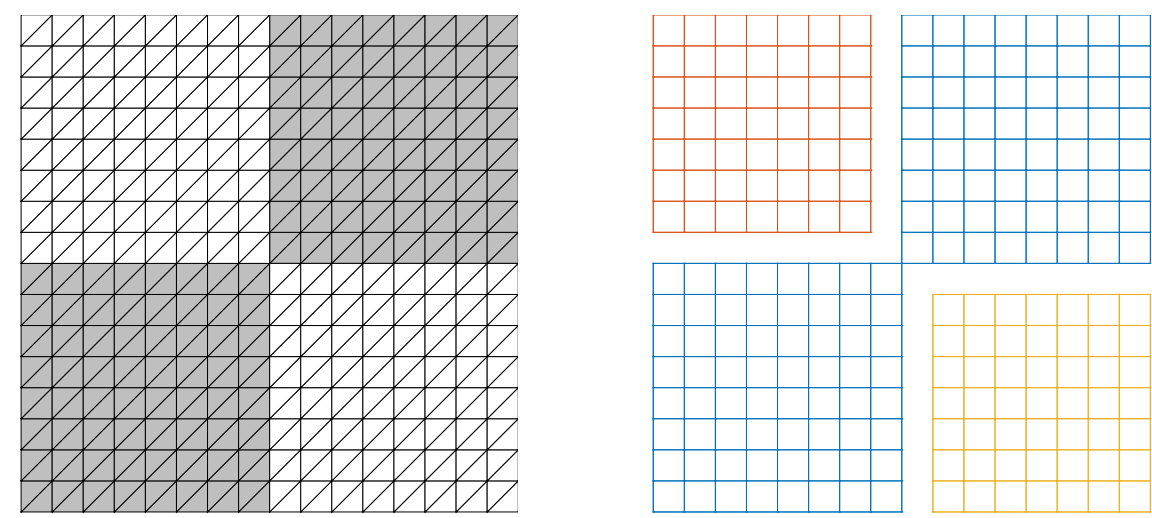

Figure 14.14. Jump coefficient problem on a uniform mesh. Left: the shaded region the coefficient is 1 , blank region the coefficient is $10^{-3}$. Right: the strongly connected components in the graph corresponding to the strength matrix $S$.

We define an operator $A_{S}: V \mapsto V^{\prime}$ using $A$ and $S$

$$
\left(A_{S} u, v\right):=\sum_{S_{i j} \neq 0} \omega_{i j}\left(u_{i}-u_{j}\right)\left(v_{i}-v_{j}\right),
$$

where $\omega_{i j}=\left|a_{i j}\right|$. We have the following lemma.

Lemma 14.1. For all $v \in V$, we have

$$
\left(A_{S} v, v\right) \leq\left(A_{+} v, v\right) \lesssim(A v, v) .
$$



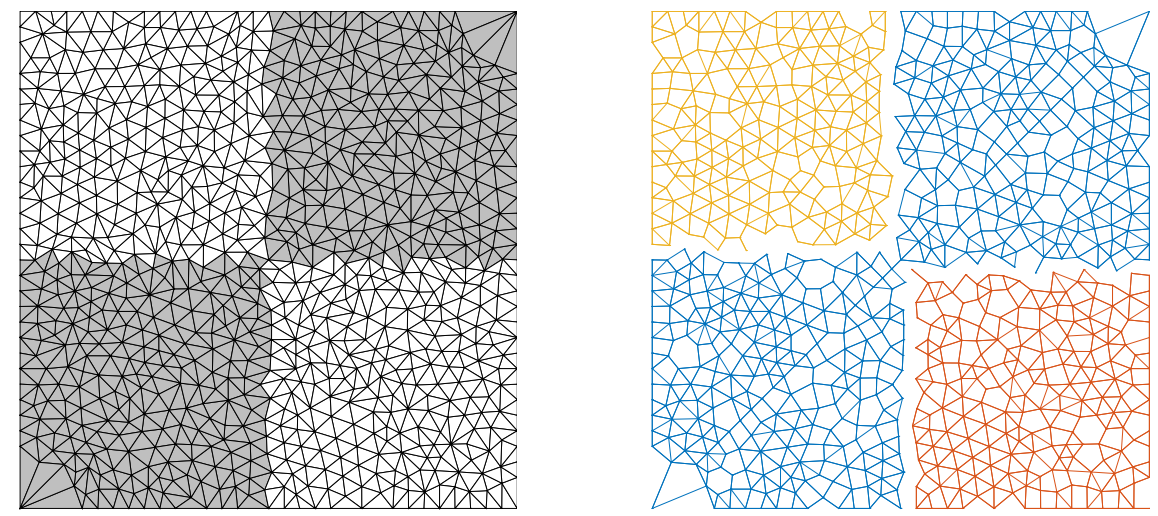

Figure 14.15. Unstructured mesh: jump coefficient problem on an unstructured mesh. Left: the shaded region the coefficient is 1 , blank region the coefficient is $10^{-3}$. Right: the strongly connected components in the graph corresponding to the strength matrix $S$.
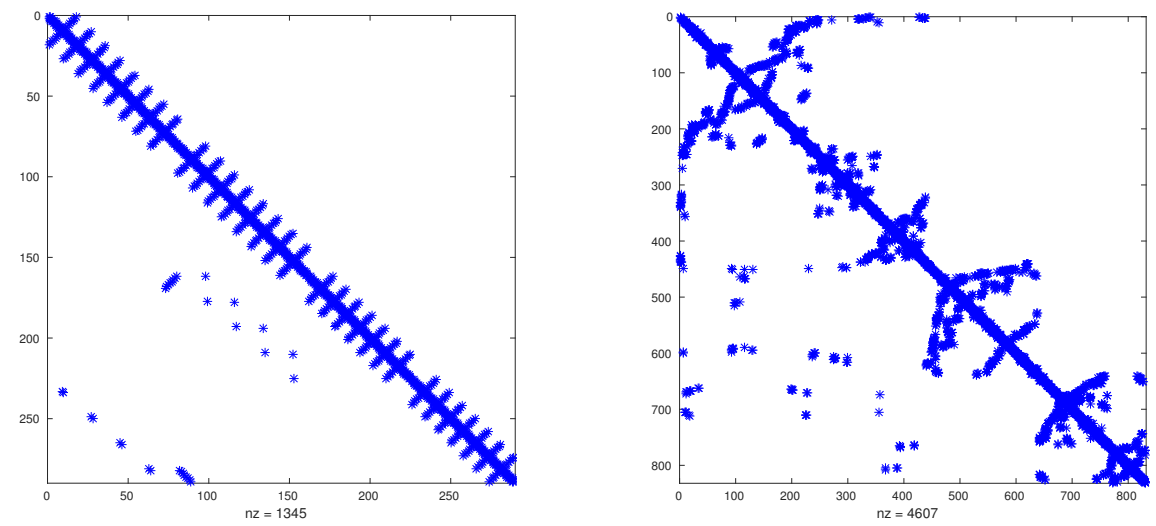

Figure 14.16. Jump coefficient problem: Nonzero pattern of strength matrix $S$ when reordered in block lower triangular form. Structured uniform grid (left) and unstructured mesh (right)

For the classical AMG, the definition of the prolongation matrix $P$ only uses strong connections, and the coarse space is union of the coarse spaces in each strongly connected component of the graph of $A_{+}$. Note that the classical AMG construction gives the same coarse space for both $A$ and its $M$-matrix relative $A_{+}$. For this reason, in the considerations that follow we write $A$ instead of $A_{+}$and $\mathcal{E}$ instead of $\mathcal{E}_{+}$, as the coarse space is defined using only entries from $A_{+}$.

We now consider the convergence of classical two-level AMG with standard interpolation for the jump coefficient problem and we prove a uniform convergence result for the two level method. The same result for direct interpolation can be obtained by a slight modification of the proof for standard interpolation case. Be- 
fore we go through the AMG two-level convergence proof, we first introduce the following result on a connected graph, which can be viewed as a discrete version of Poincaré inequality.

Lemma 14.2. We consider the following graph Laplacian on a connected undirected graph $\mathcal{G}=(\mathcal{V}, \mathcal{E})$

$$
\langle A u, v\rangle=\frac{1}{2} \sum_{(i, j) \in \mathcal{E}}\left(u_{i}-u_{j}\right)\left(v_{i}-v_{j}\right) .
$$

For any $v \in V$, the following estimate is true

$$
\left\|v-v_{c}\right\|_{\ell^{2}}^{2} \leq \mu n^{2} d\langle A v, v\rangle,
$$

where $n=|\mathcal{V}|$ is the size of the graph, $v_{c}=\sum_{j=1}^{n} w_{j} v_{j}$ is a weighted average of $v$, $\mu=\sum_{j=1}^{n} w_{j}^{2}$, and $d$ is the diameter of the graph.

We now consider the Classical AMG coarsening as defined in $\$ 12.4$ and with an abuse of notation, we use $\Omega_{j}$ to denote the set of vertices defined via the $C / F$ splitting in (12.21). Next Lemma is a spectral equivalence result, showing that the local operators $A_{j}$, defined in (12.28), for shape regular mesh, are spectrally equivalent to a scaling of the graph Laplacian operators $A_{L, j}$ defined as

$$
\left(A_{L, j} u, v\right)=\frac{1}{2} \sum_{(i, k) \in \Omega_{j}}\left(u_{i}-u_{k}\right)\left(v_{i}-v_{k}\right) .
$$

Lemma 14.3. With the assumption we made on the shape regularity of the finite element mesh, the following inequalities hold for $A_{j}$ defined as in (12.28) using the standard interpolation

$$
c_{L} h^{d-2}\left\langle A_{L, j} v_{j}, v_{j}\right\rangle \leq\left(A_{j} v_{j}, v_{j}\right) \leq c^{L} h^{d-2}\left\langle A_{L, j} v_{j}, v_{j}\right\rangle,
$$

where $A_{L, j}$ is a graph Laplacian defined in (14.1) on the graph $\mathcal{G}_{j}, h$ is the mesh size and $c_{L}, c^{L}$ are constants depend on the shape regularity constant, and the threshold $\theta$ for the strength of connections.

Proof. By the definition of the strength of connection, we have

$$
a_{i i}=\sum_{k \in N_{k}}-a_{i k} \leq-\frac{\left|N_{i}\right|}{\theta} a_{i j},
$$

Since $A$ is symmetric, we also have

$$
a_{i i} \leq-\frac{\left|N_{i}\right|}{\theta} a_{j i}
$$

By the definition of $\Omega_{j}$ in standard interpolation, for any $i \in \Omega_{j} \backslash\{j\}$, either $i \in F_{j}^{s}$ or there exists a $i \in F_{j}^{s}$ such $i \in F_{k}^{s}$. For the latter, $(j, k, i)$ forms a path between $j$ 


\subsection{Anisotropic problem}

and $i$ going along strong connections. We have then

$$
-a_{i k} \geq-\frac{\theta}{\left|N_{k}\right|} a_{k k} \geq-\frac{\theta}{\left|N_{k}\right|} a_{k j} \geq-\frac{\theta^{2}}{\left|N_{k}\right|\left|N_{j}\right|} a_{j j}
$$

and

$$
a_{j j} \geq-a_{j k} \geq \frac{\left|N_{k}\right|}{\theta} a_{k k} \geq-\left(\frac{\left|N_{k}\right|}{\theta}\right)^{2} a_{i k}
$$

Combining the above two inequalities and using the assumption that the mesh is shape regular, for any $l \in \Omega_{j}$ that is connected with $i$ we have

$$
\sigma_{1} a_{i j} \leq-a_{i l} \leq \sigma_{2} a_{j j}
$$

with constants $\sigma_{1}$ and $\sigma_{2}$ which depend on the shape regularity constant and $\theta$.

Since in the definition of $A_{j}$ in (12.28), $\omega_{e}=-a_{i j} / 2$ for $e=(i, j)$, we obtain

$$
c_{1} a_{j j}\left\langle A_{L, j} v_{j}, v_{j}\right\rangle \leq\left(A_{j} v_{j}, v_{j}\right) \leq c_{2} a_{j j}\left\langle A_{L, j} v_{j}, v_{j}\right\rangle .
$$

Then by a scaling argument, $a_{j j} \equiv h^{d-2}$ and the proof is complete. $\square$

Theorem 14.4. The two level method using a coarse space defined as $V_{c}$ defined via the classical AMG is uniformly convergent.

Proof. By Theorem 5.2, we only need to show that $\mu_{c}$ is bounded, which can be easily obtained by combining Lemma $14.2-14.3$ with Lemma 7.3 .

\subsection{Anisotropic problem}

We consider the following problem

$$
\begin{cases}-u_{x x}-\varepsilon u_{y y}=f, & \text { in } \Omega, \\ \frac{\partial u}{\partial n}=0, & \text { on } \partial \Omega .\end{cases}
$$

We discretize the problem using finite element method on an $n \times n$ uniform triangular grid in $\Omega=(0,1) \times(0,1)$. We order the vertices of the triangulation lexicographically and denote them by $\{(i h, j h)\}_{i, j=0}^{n}$. The stiffness then matrix is

$$
A=\operatorname{diag}(-\varepsilon I, B,-\varepsilon I) \quad \text { with } \quad B=\operatorname{diag}(-1,2(1+\varepsilon),-1) .
$$

It is immediate to see that, for sufficiently small $0<\varepsilon \ll 1$ the strength operator $S$ has the form:

$$
S=\operatorname{diag}\left(0, S_{B}, 0\right) \quad \text { with } \quad S_{B}=\operatorname{diag}(1,1,1) .
$$

and the $M$-matrix relative of $A$ is

$$
A_{+}=\operatorname{diag}\left(0, B_{+}, 0\right) \quad \text { with } \quad B_{+}=\operatorname{diag}(-1,2,-1) .
$$

If we use uniform coarsening to solve above linear problem, it is proved in ( $\mathrm{Yu}$, $\mathrm{Xu}$ and Zikatanov 2013) (see also (Zikatanov 2008)) that uniform convergence is 
not achieved when using point relaxation as a smoother and standard coarsening. A fix for this is to use strength of connection and coarsen the adjacency graph of the strength operator $S$. The $C / F$ splitting constructed in this way using the MIS algorithm from $\$ 9.3 .1$ results in semi-coarsening (coarsening only in one direction).

We now move on to show uniform convergence of the two-level classical AMG for the anisotropic problem (14.6). Recall that we consider a uniform grid in $\mathbb{R}^{2}$ with lexicographical ordering of the vertices. The stiffness then matrix is (14.7). We further assume, without loss of generality, that $n=2 m+1$ for some $m$. The coarse grid points using the strength operator defined in (14.8) then are with coordinates $((2 i) h, j h)$, where $i=1: m$ and $j=1: n$.

Further, as each coarse grid function is uniquely determined by its values at the coarse points, the function that corresponds to the point $((2 i) h, j h)$ for some $i$ and $j$ is defined as the unique piece-wise linear function $\phi_{i, j, H}$ satisfying:

$$
\phi((2 i) h, j h)=1, \quad \phi((2 i-1) h, j h)=1 / 2, \quad \phi((2 i+1) h, j h)=1 / 2,
$$

and $\phi_{i, j, H}(x)=0$ at all other grid nodes.

Or we can use the basis functions for bilinear element, which can be written as tensor product. Let us define first the piece-wise linear basis in 1D:

$$
\phi_{j, h}(t)=\left\{\begin{array}{l}
\frac{(t-(j-2) h)}{h}, \quad t \in((j-2) h,(j-1) h) \\
\frac{(j h-t)}{h}, \quad t \in((j-1) h, j h), \\
0, \quad \text { otherwise }
\end{array}\right.
$$

The basis in $V_{h}$ then is

$$
\phi_{i j, h}(x, y)=\phi_{i, h}(x) \phi_{j, h}(y)
$$

and for the coarse grid basis we have

$$
\phi_{i j, H}(x, y)=\phi_{i, 2 h}(x) \phi_{j, h}(y) .
$$

The basis functions for the linear elements is the piece-wise linear interpolation of the bilinear basis.

The subset $\Omega_{i j}$ is the support of this basis function, i.e. the grid points where $\phi_{i j, H}$ is non-zero. More precisely,

$$
\Omega_{i j}=\{((2 i-1) h, j h),(2 i h, j h),((2 i+1) h, j h)\} .
$$

$\Omega_{i j}$ consists of the coarse grid point $(2 i h, j h)$ and its neighbors on $x$ direction. Then we define $V_{j}:=\mathbb{R}^{3}$.

The operator $\Pi_{j}: V_{j} \mapsto V$ is defined by the matrix representation of $I_{h}\left(\phi_{i j, H^{*}}\right)$. $A_{i j}$ is defined as in (12.28). In this case

$$
A_{i j}=\left(\begin{array}{ccc}
1 & -1 & 0 \\
-1 & 2 & -1 \\
0 & -1 & 1
\end{array}\right)
$$


$A_{i j}$ is symmetric positive semi-definite and since for any $v_{i j} \in V_{i j}$, we have

$$
\begin{aligned}
\sum_{i, j}\left(v_{i j}, v_{i j}\right)_{A_{i j}} & =\sum_{i j} \sum_{\substack{(k, l) \in \mathcal{E} \\
k, l \in \Omega_{i j}}}-a_{k l}\left(v_{k}-v_{l}\right)^{2} \\
& \leq 2 \sum_{(k, l) \in \mathcal{E}}-a_{k l}\left(v_{k}-v_{l}\right)^{2} \\
& =2(v, v)_{A},
\end{aligned}
$$

$A_{i j}$ satisfies 6.11).

We define $D_{i j}$ as in 12.30 . As $A_{i j} \mathbf{1}=\mathbf{0}$, the minimum eigenvalue of $D_{i j}^{-1} A_{i j}$ is 0 and the corresponding eigenvector is the constant vector, we choose local coarse space $V_{i j}^{c}$ to be the space of all constant vectors in $V_{i j}$. The corresponding global coarse space is then defined as in 6.13.

Theorem 14.5. The two level method with coarse space defined above converges uniformly for the anisotropic problem in (14.6), with convergence rate independent of $\varepsilon$ and the mesh size $h$.

Proof. By Theorem 5.2, the convergence rate depends on the second smallest eigenvalue of $D_{i j}^{-1} A_{i j}$ which is 1 for all $i$ and $j$.

Next, Theorem 5.2 can be applied to this case, and we obtain

$$
\|E\|_{A} \leq 1-\frac{1}{C}
$$

with $C$ independent of $\varepsilon$ and the mesh size, which proves the uniform convergence of the AMG method.

\subsection{Bibliographical notes}

Fast solvers for problems with heterogeneous and or anisotropic coefficients have been in the focus of research for the last 3-4 decades. The AMG methods are among the preferred solvers due their robust behavior with respect to the coefficient variation and independence of the geometry. Standard multilevel methods for these problems do have limitations as their convergence may deteriorate as shown in (Alcouffe, Brandt, Dendy and Painter 1981). The cause for this in geometric multigrid with standard coarse spaces is discussed in (Bramble and $\mathrm{Xu}$ 1991, Xu 1991) and later in (Oswald 1999). Attempts to remove the dependence on the size of the coefficient jumps was made by introducing the matrix dependent prolongations. We refer to (Dendy 1982, Dendy 1983), Reusken (Reusken 1993), (de Zeeuw 1990). Several theoretical and numerical results on geometric and algebraic methods for discontinuous coefficients are found in the survey paper (Chan and Wan 2000) and the references therein. Anisotropic equations and AMG coarsening is considered in (Brannick, Brezina, MacLachlan, Manteuffel, McCormick and Ruge 2006). 
Finally, in addition to the theory presented here, some partial theoretical results on convergence of an AMG are found in the classical papers on AMG (Ruge and Stüben 1987), smoothed aggregation (SA) (Vaněk et al. 1996b). Related works are the frequency filtering and decompositions found in (Hackbusch 1989), (Wittum 1992, Wagner and Wittum 1997, Weiler and Wittum 1997, Nägel, Falgout and Wittum 2008).

\section{Bootstrap and adaptive AMG}

In all the algorithms studied earlier, we assume that the near-null spaces are known in advance. Eliminating such an assumption and extending the range of applicability of optimal multigrid techniques is attempted in the framework of the Bootstrap/Adaptive AMG methods which we describe in this section. In summary, the adaptive AMG ( $\alpha$ AMG, $\alpha \mathrm{SA}$ ) and bootstrap AMG (BAMG) algorithms are aimed at the solution of harder problems for which the standard variants of the Classical AMG method or the Smoothed Aggregation method may converge slow. The bootstrap/adaptive algorithms make special choices coarse spaces. Based on a given a smoother. self- improve until achieving the desired convergence rate.

\subsection{Sparsity of prolongation matrices}

We give here a very short summary on the sparsity patterns of the prolongation matrices that we have defined for energy minimization AMG in \$11. Classical AMG in $\$ 12$, and Aggregation AMG in $\$ 13$. First, for the energy minimizing AMG we have that the sparsity pattern of the prolongation could be prescribed in advance, and in some sense this approach is more general as it can also use the sparsity patterns given below for the Classical AMG and Aggregation AMG approaches. For specific restrictions related to the number of vectors interpolated exactly by the energy minimizing prolongation we refer to (Xu and Zikatanov 2004) and (Vassilevski and Zikatanov 2006). There are also ways to define the prolongation with changing pattern. Such algorithms are useful because they provide a mechanism to control the sparsity pattern of the prolongation. The Algorithm 7 is first described in (Brandt 2002) and later included in the bootstrap adaptive AMG algorithm designed in (Brandt, Brannick, Kahl and Livshits 2011a). In the algorithm description, we refer to the graph of the $M$-matrix relative as defined in $\$ 9$. 


\subsection{Notation}
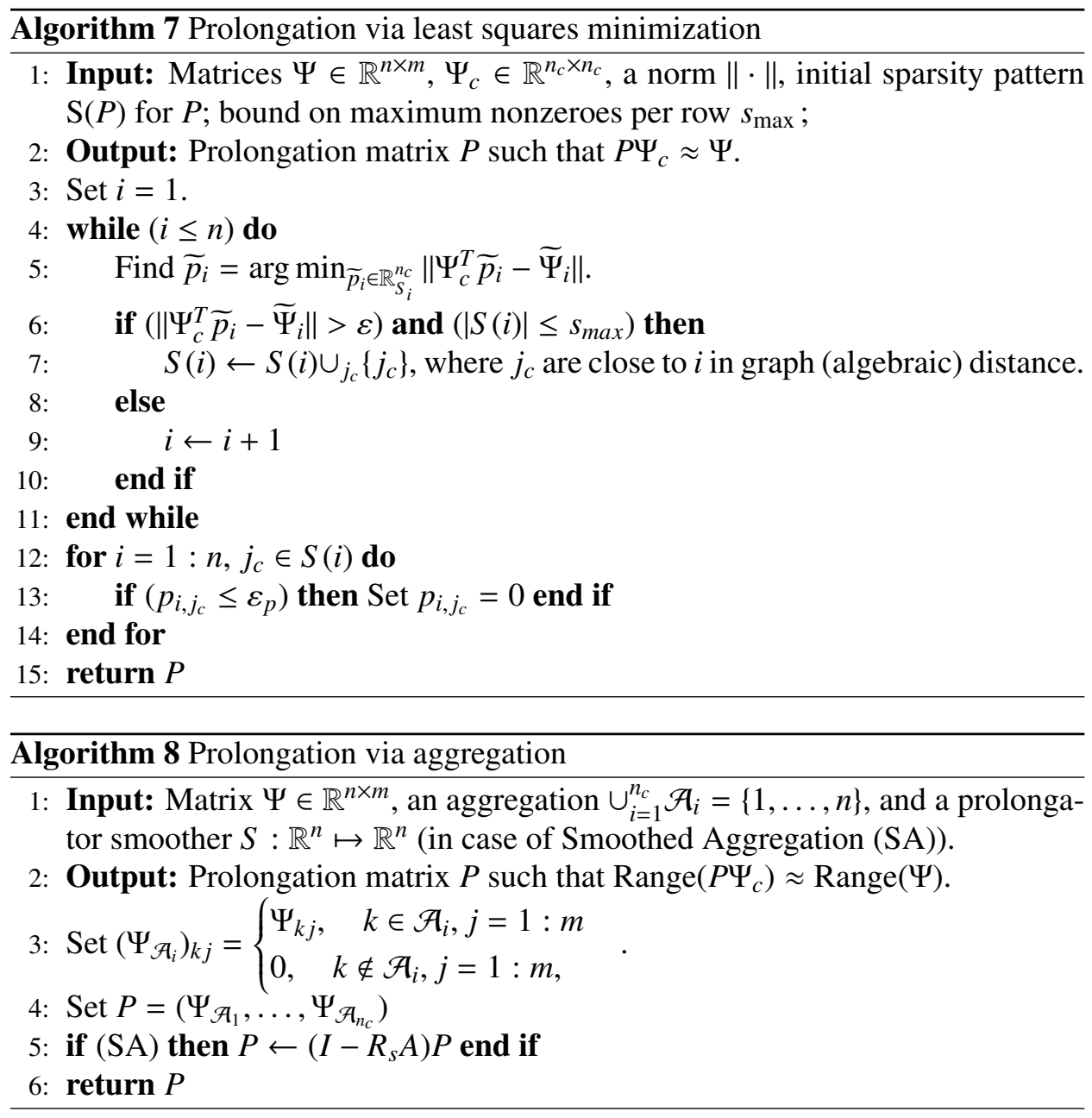

\subsection{Notation}

Given a matrix $A \in \mathbb{R}^{n \times n}$ and a relaxation (smoother) $R$ for this matrix, adaptive procedure to construct a sequence of coarse spaces which are characterized by the sequence of prolongation matrices

$$
P_{j}^{m}: \mathbb{R}_{j}^{n_{j}^{m}} \mapsto \mathbb{R}_{j+1}^{n_{j+1}^{m}}
$$

The corresponding V-cycle matrix is denoted $B^{m}$. We introduce the following notation, used throughout this section

- For a matrix $Y \in \mathbb{R}^{n \times m}$ we set

$$
Y=\left(y_{1}, \ldots, y_{m}\right)=\left(\begin{array}{c}
\widetilde{y}_{1}^{T} \\
\vdots \\
\widetilde{y}_{n}^{T}
\end{array}\right)
$$


Clearly, here $\left\{y_{i}\right\}_{i=1}^{m}$ are the columns of $Y$ and $\left\{\tilde{y}_{j}^{T}\right\}_{j=1}^{n}$ are the rows of $Y$.

- $\mathcal{V}=\left(V_{1}, \ldots V_{J}\right)$ is a multilevel hierarchy of spaces $V_{j} \subset V_{j+1}, j=1:(J-1)$ and the fine grid spaces is denoted by $V_{J}$.

- By $\left\{P_{j-1}^{j}\right\}_{j=2}^{J}$ we denote the prolongation matrix from a coarser level $(j-1)$ to finer level $j$, and $P_{j}=P_{J-1}^{J} P_{J-2}^{J-1} \ldots P_{j}^{j+1}, j=1, \ldots(J-1)$ is the prolongation from level from level $j$ to level $J$

- The set of all prolongations up to level $j$ we denote by $\mathcal{P}_{j}=\left\{P_{j-1}^{j}\right\}_{j=2}^{j}$, and $\mathcal{P}=\mathcal{P}_{J}$. As we have mentioned on several occasions, the set of prolongation matrices $\mathcal{P}$ completely determines the multilevel hierarchy of spaces $\mathcal{V}$.

- Set of test vectors on every level

$$
C=\left\{\Psi_{1}, \cdots, \Psi_{J}\right\}, \quad \Psi_{j} \in \mathbb{R}^{n_{j} \times m} .
$$

In an adaptive method, the hierarchy of coarse spaces is constructed so that $\Psi_{j} \approx P_{j-1}^{j} \Psi_{j-1}$, or, $P_{j-1}^{j}$ is constructed so that $\Psi_{j}$ can be well approximated by elements from Range $P_{j-1}^{j}$.

- We need the standard $V$-cycle preconditioner $B\left(\mathcal{P}_{j}\right)$ with hierarchy of spaces given by $\mathcal{P}_{j}$ starting at level $j$.

- $A_{j}$ is the restriction of the fine grid matrix $A$ on level $j$ and $M_{j}$ is the restriction of the "mass" matrix, defined as: $A_{j}=P_{j}^{T} A P_{j}, M_{j}=P_{j}^{T} P_{j}$.

A generic adaptive AMG algorithm changes the set $\mathcal{P}$, adjusting the hierarchy of spaces $\mathcal{V}$. In general, in adaptive procedure the number of levels is not known and in some of the algorithms below we use $V_{1}$ as the finest space by mapping the indexes in the notations above as

$$
j \leftarrow J-j+1, \quad j=1, \ldots J .
$$

\subsection{A basic adaptive algorithm}

We describe now a generic Adaptive algorithm for constructing coarse spaces. We use an approach slightly different than what is in the literature and fit into one framework both BAMG and $\alpha \mathrm{SA}$.

Step 0 Given $A \in \mathbb{R}^{n \times n}$ and the associated graph $\mathcal{G}(A)=(\mathcal{V}, \mathcal{E})$.

Step 1 Initialization

1. Given $m_{0} \geq 1, q \geq 1,1 \leq n_{0}<n$ and $\delta_{0} \in(0,1)$.

2. $\mathcal{P} \leftarrow \emptyset$.

3. $V_{c} \leftarrow V=\mathbb{R}^{n} ; \mathcal{V} \leftarrow\left\{V_{c}\right\}$.

4. $n_{c} \leftarrow n ; m \leftarrow m_{0}$.

5. $B \leftarrow R$.

6. Randomly pick $m$ test-vectors $\boldsymbol{\Psi}_{0}=\left(\psi_{1}, \ldots \psi_{m}\right), \Psi_{0} \in \mathbb{R}^{n \times m}$.

7. $\boldsymbol{\Psi} \leftarrow \boldsymbol{\Psi}_{0} ; C \leftarrow\left\{\boldsymbol{\Psi}_{0}\right\}$.

Step 2 If $n_{c} \leq n_{0}$, go to Step 3, else do: 


\subsection{Bibliographical notes}

1 Make a copy of $\boldsymbol{\Psi}: \hat{\Psi} \leftarrow \Psi$. Then compute

$$
\Psi \leftarrow(I-B A)^{q} \Psi, \quad \delta=\max _{1 \leq i \leq m} \frac{\left\|\Psi_{i}\right\|_{A}}{\left\|\hat{\Psi}_{i}\right\|_{A}} .
$$

2 If $\delta \leq \delta_{0}$ then Stop.

3 Use a coarsening strategy (c.f. $\$ 9$ ) to update $n_{c}$ and find a set of coarse grid DOFs $\left\{N_{i}(\cdot)\right\}_{i=1}^{n_{c}}$. Then set

$$
V_{c} \leftarrow \mathbb{R}^{n_{c}}, \quad \mathcal{V} \leftarrow \mathcal{V} \cup\left\{V_{c}\right\}
$$

4 Form the "restriction" of $\boldsymbol{\Psi}$ to the coarse space:

$$
\Psi_{c} \leftarrow \operatorname{Restrict}\left(\Psi,\left\{N_{i}(\cdot)\right\}_{i=1}^{n_{c}}\right)
$$

Then set $C \leftarrow C \cup\left\{\Psi_{c}\right\}$.

5 Identify a sparsity pattern $\mathrm{S}(P)$ (c.f. $\$ 15.1$ also $\$ 12.3$, and $\$ 13.2$ ).

6 Find a prolongation matrix $P$ using $\Psi_{c}$ and $\Psi$ by applying Algorithm 7 or Algorithm 8, Then set

$$
\mathcal{P} \leftarrow \mathcal{P} \cup\{P\}, \quad A \leftarrow P^{T} A P, \quad \Psi \leftarrow \Psi_{c}, B \leftarrow R_{c},
$$

where $R_{c}$ is the relaxation on the coarse grid $V_{c}$.

Step 3 Order the spaces in $\mathcal{V}$ increasing with respect to their dimension as

$$
\mathcal{V}=\left\{V_{1}, V_{2}, \ldots, V_{J}\right\} \text { with } V_{J}=\mathbb{R}^{n},
$$

and the corresponding prolongation matrices

$$
\mathcal{P}=\left\{P_{j}^{j+1}\right\}_{j=1}^{J-1}, \quad P_{j}^{j+1}: V_{j} \mapsto V_{j+1}, \quad j=1,2, \ldots, J-1 .
$$

Set $B$ to be the $V$-cycle AMG method based on above coarse spaces and prolongation matrices.

Step 4 Compute $\delta$ in (15.1) using current $B$. If $\delta \leq \delta_{0}$, Stop. Else, update $C$, $\mathcal{P}$ and $\mathcal{V}$ by modifying, removing from, or adding to the set of test-vectors (Bootstrap AMG uses Algorithm 9, adaptive SA uses Algorithm 10]. Then go to Step 3 .

The restrict operator in Step 2.4 is following:

$$
\begin{array}{r}
\boldsymbol{\Psi}_{c}=\left(N_{i}\left(\psi_{j}\right)\right)_{1 \leq i \leq n_{c}, 1 \leq j \leq m}, \quad \text { bootstrap AMG } \\
\Psi_{c}=\left(N_{i}\left(\psi_{j}\right)\right)_{1 \leq i \leq n_{c}} \otimes e_{j} \quad j=1: m, \quad \text { adaptive aggregation }
\end{array}
$$

\subsection{Bibliographical notes}

The AMG approaches we have considered are aimed at adaptive choice of coarse spaces and multilevel hierarchies in an AMG algorithm. The majority of known to date adaptive AMG methods use the operator $A$ and aim to capture the worst 


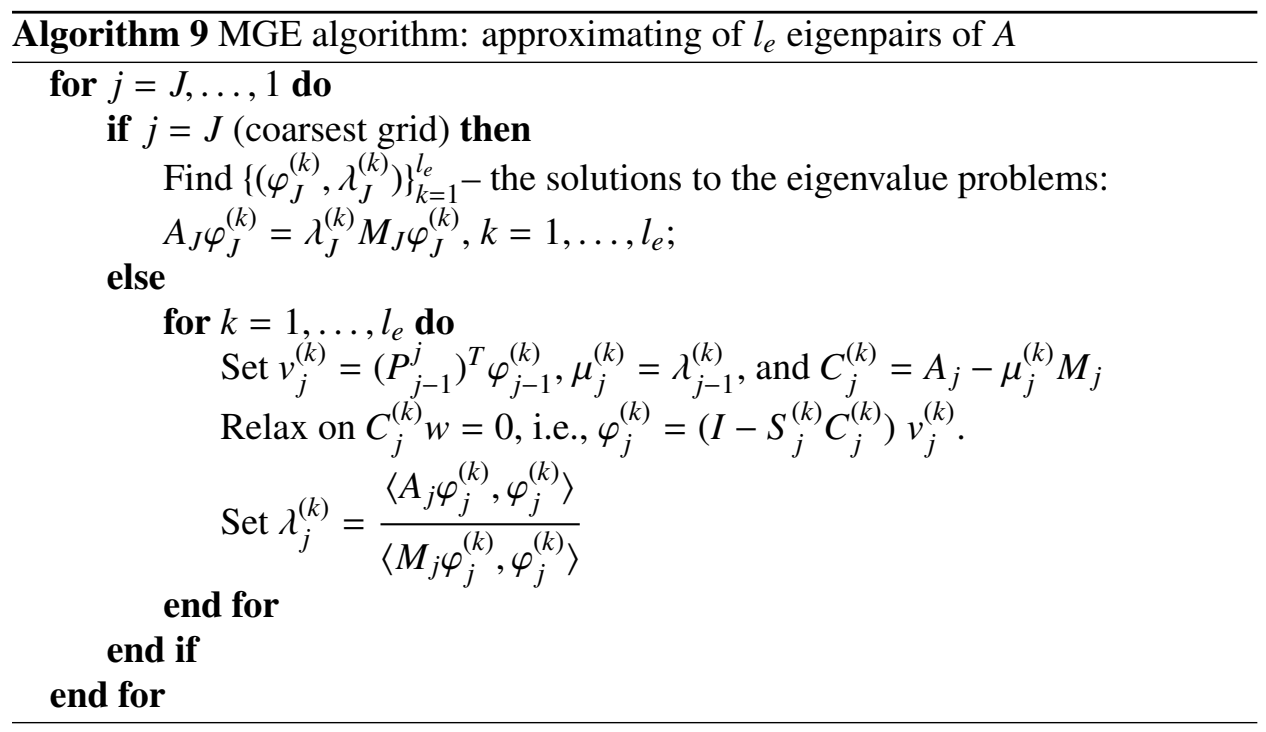

case errors. All particular details regarding bootstrap AMG (BAMG) are found in (Brandt 2002), (Brandt et al. 2011a), and (Brandt, Brannick, Kahl and Livshits 2015). The Adaptive Classical AMG is described in adaptive AMG or $\alpha A M G$ (Brezina et al. 2006b), and the adaptive Smoothed Aggregation or $\alpha S A$ is discussed in detail in (Brezina, Falgout, MacLachlan, Manteuffel, McCormick and Ruge 2004b), (Brezina, Falgout, MacLachlan, Manteuffel, McCormick and Ruge 2005a). While the adaptive and bootstrap MG processes have been successful in several settings, they are still only a heuristic attempt to overcome serious barriers in achieving good performance from the classical AMG point of view. Indeed, the costs of achieving added robustness using a bootstrap or adaptive $\mathrm{MG}$ algorithm are significant.

Central to the adaptive MG framework is an important distinction between the role of the underlying multigrid algorithm (aggregation or classical AMG) and what the additional, adaptive elements it should provide. If the idea of self-improving the coarse spaces is poorly implemented, the adaptive and bootstrap multigrid algorithms can easily degenerate into an algorithm with no better convergence properties than a classical Krylov method preconditioned by the MG smoother (Falgout 2004).

The basic ideas on adaptive AMG are outlined in the early works on classical AMG (Brandt et al. 1982b). In fact, the adaptive process of constructing interpolation based on fitting a set of test vectors for badly scaled matrices was introduced as early as in (Ruge 1983). Some of the basic ideas of adaptive AMG are also found in (Ruge 1985, Ruge 1986, Mccormick and Ruge 1989, Brandt et al. 1985). Further advancement in the adaptive AMG methods, and new ideas for using eigenvector approximations to guide the adaptive process were introduced in the bootstrap AMG (BAMG) framework in (Brandt 2001). The BAMG is a self-learning multigrid algorithm that automatically determines the algebraically smooth errors 
Algorithm 10 $\alpha$ SA: adding a test vector $\psi$ to the current set of test vectors $\Psi_{J}$

Step 1 Update $\boldsymbol{\Psi}_{J}$ by adding $\psi$ as a new column: $\boldsymbol{\Psi}_{J} \leftarrow\left[\boldsymbol{\Psi}_{J}, \psi\right]$.

Step 2 For $l=J, \ldots, 3$ :

(1) Update $\Psi_{l-1}$ by evaluating the coarse grid degrees of freedom $N_{j}(\psi), j=$ $1: n_{l-1}$. This process adds $n_{l-1}$ rows to $\Psi_{l-1}$.

(2) Use Algorithm 8 (SA) to define a prolongation $P_{l-1}^{l}$ matrix and coarse grid operator $A_{l-1}$.

(3) Update $\mathcal{P}_{l} \leftarrow\left\{P_{l-1}^{l}\right\} \cup \mathcal{P}_{l-1}$.

(4) As the number of rows in $\Psi_{l-1}$ was increased in Step 2(1), we need to change $P_{l-2}^{l-1}$ in $\mathcal{P}_{l-1}$ in order to keep the set $\mathcal{P}$ consistent. We define $\widetilde{P}_{l-2}^{l-1}$ as the "bridge" prolongation via algorithm 11 and we set

$$
\widetilde{\mathcal{P}}_{l-1}=\leftarrow\left\{\widetilde{P}_{l-2}^{l-1}\right\} \cup \mathcal{P}_{l-2}
$$

(5) Test the convergence of $B\left(\widetilde{\mathcal{P}}_{l-1}\right)$ on the newly added test vector $\psi_{l-1}$ :

$$
\hat{\psi}_{l-1} \leftarrow \psi_{l-1}, \quad \psi_{l-1} \leftarrow\left(I-B_{l-1}\left(\widetilde{\mathcal{P}}_{l-1}\right) A\right)^{q}\left(\psi_{l-1}\right) .
$$

If $\left(\frac{\left\|P_{l-1} \psi_{l-1}\right\|_{A}^{2}}{\left\|P_{l-1} \widehat{\psi}_{l-1}\right\|_{A}^{2}}\right)^{1 / q} \leq \delta$ then Stop.

(6) Update the coarse representation of $\Psi_{l-1}$ :

$$
\boldsymbol{\Psi}_{l-1} \leftarrow\left[\hat{\mathbf{\Psi}}^{l-1}, \psi_{l-1}\right] .
$$

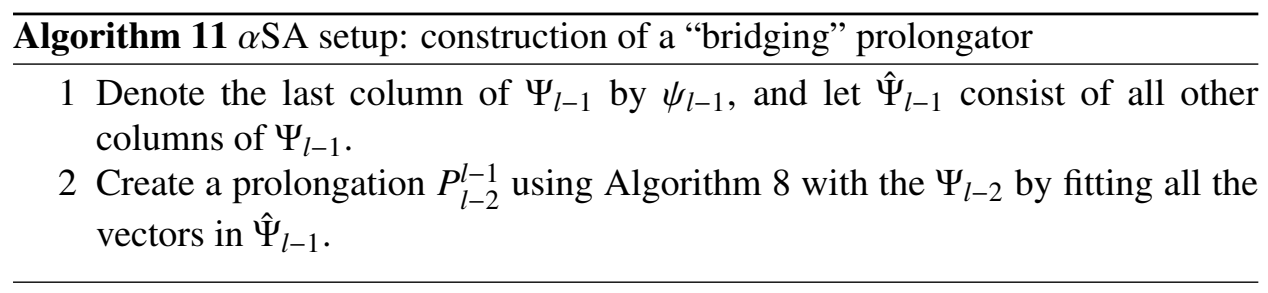

in a given problem and was further developed in (Brandt et al. 2011a), and (Brandt et al. 2015). Adaptive AMG algorithms were further developed and new were introduced in some more recent works: $(\alpha \mathrm{AMG})$ in the framework of Classical AMG (Brezina et al. 2006c); and ( $\alpha$ SA) in the framework of Smoothed Aggregation AMG (Brezina, Falgout, MacLachlan, Manteuffel, McCormick and Ruge 2004a, Brezina, Falgout, Maclachlan, Manteuffel, Mccormick and Ruge 2005b). Other adaptive multilevel algorithms are the adaptive filtering and the filtering decompositions (Wittum 1992, Wagner and Wittum 1997) and the multilevel multigraph algorithms (Bank and Smith 2002).

These references have more specific details on the implementation of BAMG $/ \alpha \mathrm{AMG} / \alpha \mathrm{SA}$. One improvement on the original BAMG method is given in (Manteuffel, McCormick, Park and Ruge 2010), where an indirect BAMG (iBAMG) method is introduced. Compared to BAMG, the iBAMG follows more closely the spirit of 
classical AMG, which attempts to collapse unwanted connections based on the assumption that the smooth error is locally constant.

In the work (Brandt, Brannick, Kahl and Livshits 2011b), BAMG is paired with an adaptive relaxation (Brandt 2000b, Kahl 2009) and a multigrid eigensolver (Brezina, Manteuffel, McCormick, Ruge, Sanders and Vassilevski 2008). A combination of the bootstrap cycling scheme (Livshits 2008, Kahl 2009) and then the multigrid eigensolver is used to compute sufficiently accurate sets of test vectors and adaptive relaxation is used to improve the AMG setup cycle.

In the recent years there have been introduced also other adaptive approaches to constructing hierarchy of spaces. Classical AMG based approach in defining the hierarchy of spaces is considered in the framework of adaptive reduction algorithms in (Brannick, Frommer, Kahl, MacLachlan and Zikatanov 2010) and (MacLachlan et al. 2006). Adaptive BoxMG was considered in (MacLachlan et al. 2012). Specialized adaptive approach for Markov Chains is presented in (De Sterck, Miller, Treister and Yavneh 2011).

\section{Concluding remarks}

In this paper, we try to give a coherent presentation of a number algebraic multigrid (AMG) methods. But this presentation, limited by our current theoretical understanding of AMG algorithms in general, is by no means complete. We choose to include those AMG algorithms that can fit into the theoretical frameworks that are presented in this paper. One notable exception is the bootstrap and adaptive AMG presented in $\$ 15$. This type of algorithms still lack a good theoretical understanding, but they provide an algorithmic framework to leverage and extend those AMG algorithms presented in $\$ 10$ 13 for a wider range of applications.

There are still many AMG algorithms in the literature that we are not able to include in this article for two main reasons. One is that those algorithms can not be easily cast within our theoretical frameworks; another is that there are algorithms that the authors are yet to comprehend on a reasonable theoretical level. Examples of algorithms and results that need further investigation and analysis include adaptive filtering, multilevel ILU methods, the multilevel convergence properties of SA-AMG, BAMG, Adaptive AMG and many others.

The AMG methods studied in this paper are obtained by optimizing the choice of coarse spaces based on a given smoother. Indeed, almost all the existing AMG method follow this strategy. It is possible, however, that an AMG method can also be designed by optimizing the choice of smoother based on a given coarsening strategy. This, in our view, is a subject worthy further investigation. Theoretically, it is also conceivable to optimize both coarsening and smoother simultaneously.

Finally, we would like to note that several AMG software packages have been developed and are currently in use, most noticeably:

Hypre: http://acts.nersc.gov/hypre/,

Trilinos: https://trilinos.org/, 
Multigraph: http://ccom.ucsd.edu/ reb/software.html

AGMG: http://homepages.ulb.ac.be/ ynotay/AGMG/,

PyAMG: http://pyamg.org/,

FASP: http://fasp.sourceforge.net/

\section{Acknowledgments}

The authors wish to thank Stephen F. McCormick, Xiaozhe Hu, James Brannick, Scott MacLachlan for many helpful discussions and suggestions and especially to thank Hongxuan Zhang for his help throughout the preparation of this article.

The work of the first author was supported by the DOE Grant DE-SC0009249 as part of the Collaboratory on Mathematics for Mesoscopic Modeling of Materials and by NSF grants DMS-1412005 and DMS-1522615. The work of the second author was supported by NSF grants DMS-1418843 and DMS-1522615.

\section{REFERENCES}

R. E. Alcouffe, A. Brandt, J. E. Dendy, Jr. and J. W. Painter (1981), 'The multigrid method for the diffusion equation with strongly discontinuous coefficients', SIAM J. Sci. Statist. Comput. 2(4), 430-454.

O. Axelsson and P. S. Vassilevski (1991), 'A black box generalized conjugate gradient solver with inner iterations and variable-step preconditioning', SIAM J. Matrix Anal. Appl. 12(4), 625-644.

O. Axelsson and P. S. Vassilevski (1994), 'Variable-step multilevel preconditioning methods I. Self-adjoint and positive definite elliptic problems', Numer. Linear Algebra Appl. 1(1), 75-101.

B. Ayuso de Dios, F. Brezzi, L. D. Marini, J. Xu and L. Zikatanov (2014), 'A simple preconditioner for a discontinuous Galerkin method for the Stokes problem', J. Sci. Comput. 58(3), 517-547.

I. Babuška and J. E. Osborn (1983), 'Generalized finite element methods: their performance and their relation to mixed methods', SIAM Journal on Numerical Analysis 20(3), 510-536.

N. S. Bahvalov (1966), 'Convergence of a relaxation method under natural constraints on an elliptic operator', Ž. Vyčisl. Mat. i Mat. Fiz. 6, 861-883.

R. E. Bank and C. C. Douglas (1985), 'Sharp estimates for multigrid rates of convergence with general smoothing and acceleration', SIAM J. Numer. Anal. 22(4), 617-633.

R. E. Bank and T. F. Dupont (1980), Analysis of a two-level scheme for solving finite element equations, Technical Report Report CNA-159, Center for Numerical Analysis, Center for Numerical Analysis, University of Texas, Austin.

R. E. Bank and R. K. Smith (2002), 'An algebraic multilevel multigraph algorithm', SIAM J. Sci. Comput. 23(5), 1572-1592 (electronic).

R. Blaheta (1986), 'A multilevel method with correction by aggregation for solving discrete elliptic problems', Aplikace matematiky 31(5), 365-378.

R. Blaheta (1988), 'Iterative methods for the numerical solution of the boundary value problem of elasticity [abstract of thesis]', Commentationes Mathematicae Universitatis Carolinae 029(1), 200-200. 


\section{CONCLUDING REMARKS}

D. Braess and W. Hackbusch (1983), 'A new convergence proof for the multigrid method including the $V$-cycle', SIAM J. Numer. Anal. 20(5), 967-975.

J. H. Bramble (1993), Multigrid methods, Vol. 294 of Pitman Research Notes in Mathematics Series, Longman Scientific \& Technical, Harlow.

J. H. Bramble and J. E. Pasciak (1987), 'New convergence estimates for multigrid algorithms', Mathematics of computation 49(180), 311-329.

J. H. Bramble and J. Xu (1991), 'Some estimates for a weighted $L^{2}$ projection', Math. Comp. 56(194), 463-476.

J. H. Bramble, J. E. Pasciak and J. Xu (1990), 'Parallel multilevel preconditioners', Mathematics of Computation 55(191), 1-22.

J. H. Bramble, J. E. Pasciak and J. Xu (1991a), 'The analysis of multigrid algorithms with nonnested spaces or noninherited quadratic forms', Math. Comp. 56(193), 1-34.

J. H. Bramble, J. E. Pasciak, J. P. Wang and J. Xu (1991b), 'Convergence estimates for multigrid algorithms without regularity assumptions', Math. Comp. 57(195), 23-45.

J. H. Bramble, J. E. Pasciak, J. P. Wang and J. Xu (1991c), 'Convergence estimates for product iterative methods with applications to domain decomposition', Math. Comp. 57(195), 1-21.

A. Brandt (1973), Multi-level adaptive technique (MLAT) for fast numerical solution to boundary value problems, in Proc. 3rd Internat. Conf. on Numerical Methods in Fluid Mechanics (Paris, 1972), Vol. 18 of Lecture Notes in Physics, Springer-Verlag, Berlin, New York, pp. 82-89.

A. Brandt (1977), 'Multi-level adaptive solutions to boundary-value problems', Math. Comp. 31(138), 333-390.

A. Brandt (1986), 'Algebraic multigrid theory: the symmetric case', Appl. Math. Comput. 19(1-4), 23-56.

A. Brandt (2000a), 'General highly accurate algebraic coarsening', Electronic Transactions on Numerical Analysis 10, 1-20.

A. Brandt (2000b), 'General highly accurate algebraic coarsening', Electon. Trans. Numer. Anal. 10(9680), 1-20.

A. Brandt (2001), 'Multiscale scientific computation: Review 2001', in Multiscalle and Multiresolution Methods: Theory and Applications, T. J. Barth, T. F. Chan, and R. Haimes, eds, 1, 1-96.

A. Brandt (2002), Multiscale scientific computation: Review 2001, in Multiscale and Multiresolution Methods: Theory and Applications (T. J. Barth, T. Chan and R. Haimes, eds), Springer Berlin Heidelberg, Berlin, Heidelberg, pp. 3-95.

A. Brandt, J. Brannick, K. Kahl and I. Livshits (2011a), 'Bootstrap AMG', SIAM J. Sci. Comput. 33(2), 612-632.

A. Brandt, J. Brannick, K. Kahl and I. Livshits (2011b), 'Bootstrap amg', SIAM J. Sci. Comput pp. 612-632.

A. Brandt, J. Brannick, K. Kahl and I. Livshits (2015), 'Bootstrap algebraic multigrid: status report, open problems, and outlook', Numer. Math. Theory Methods Appl. 8(1), 112-135.

A. Brandt, S. McCormick and J. Ruge (1985), Algebraic multigrid (AMG) for sparse matrix equations, in Sparsity and its applications (Loughborough, 1983), Cambridge Univ. Press, Cambridge, pp. 257-284.

A. Brandt, S. F. McCormick and J. W. Ruge (1982a), Algebraic multigrid (AMG) for 
automatic multigrid solutions with application to geodetic computations, Technical report, Inst. for Computational Studies, Fort Collins, CO.

A. Brandt, S. F. McCormick and J. W. Ruge (1982b), Algebraic multigrid (AMG) for automatic multigrid solutions with application to geodetic computations, Technical report, Inst. for Computational Studies, Fort Collins, CO.

J. Brannick and L. Zikatanov (2006), Algebraic multigrid methods based on compatible relaxation and energy minimization, in Domain Decomposition Methods in Science and Engineering XVI (O. B. Widlund and D. E. Keyes, eds), Springer-Verlag, Berlin, pp. 15-26.

J. Brannick, M. Brezina, S. MacLachlan, T. Manteuffel, S. McCormick and J. Ruge (2006), 'An energy-based AMG coarsening strategy', Numerical Linear Algebra with Applications 13, 133-148.

J. Brannick, Y. Chen and L. Zikatanov (2012), 'An algebraic multigrid method for anisotropic elliptic equations based on subgraph matching equations based on subgraph matching', Numerical Linear Algebra with Applications 19, 279 - 295.

J. Brannick, A. Frommer, K. Kahl, S. MacLachlan and L. Zikatanov (2010), 'Adaptive reduction-based multigrid for nearly singular and highly disordered physical systems', Electron. Trans. Numer. Anal. 37, 276-295.

J. J. Brannick and R. D. Falgout (2010), 'Compatible relaxation and coarsening in algebraic multigrid', SIAM J. Sci. Comput. 32(3), 1393-1416.

M. Brezina and P. Vassilevski (2011), Smoothed aggregation spectral element agglomeration AMG: SA- $\rho$ AMGe., in LSSC (I. Lirkov, S. Margenov and J. Wasniewski, eds), Vol. 7116 of Lecture Notes in Computer Science, Springer, pp. 3-15.

M. Brezina, A. Cleary, R. Falgout, V. Henson, J. E. Jones, T. Manteuffel, S. McCormick and J. Ruge (2001), 'Algebraic Multigrid Based on Element Interpolation (AMGe)', SIAM Journal on Scientific Computing 22(5), 1570-1592.

M. Brezina, R. Falgout, S. MacLachlan, T. A. Manteuffel, S. F. McCormick and J. Ruge (2004a), 'Adaptive smoothed aggregation ( $\alpha$ SA)', SIAM J. Sci. Comp. 25(6), 18961920.

M. Brezina, R. Falgout, S. MacLachlan, T. Manteuffel, S. McCormick and J. Ruge (2004b), 'Adaptive smoothed aggregation ( $\alpha S A)$ ', SIAM J. Sci. Comput. 25(6), 1896-1920.

M. Brezina, R. Falgout, S. MacLachlan, T. Manteuffel, S. McCormick and J. Ruge (2005a), 'Adaptive smoothed aggregation ( $\alpha \mathrm{SA})$ multigrid', SIAM Rev. 47(2), 317-346.

M. Brezina, R. Falgout, S. Maclachlan, T. Manteuffel, S. Mccormick and J. Ruge (2005b), 'Adaptive smoothed aggregation $(\alpha \mathrm{SA})$ multigrid', SIAM Rev. 47(2), 317-346.

M. Brezina, R. Falgout, S. MacLachlan, T. Manteuffel, S. McCormick and J. Ruge (2006a), 'Adaptive algebraic multigrid', SIAM J. Sci. Comput. 27(4), 1261-1286.

M. Brezina, R. Falgout, S. MacLachlan, T. Manteuffel, S. McCormick and J. Ruge (2006b), 'Adaptive algebraic multigrid', SIAM J. Sci. Comput. 27(4), 1261-1286.

M. Brezina, R. Falgout, S. MacLachlan, T. Manteuffel, S. McCormick and J. Ruge (2006c), 'Adaptive algebraic multigrid ( $\alpha \mathrm{amg})$ ', SIAM J. Sci. Comp. 27, 1261-1286.

M. Brezina, T. Manteuffel, S. McCormick, J. Ruge, G. Sanders and P. Vassilevski (2008), 'A generalized eigensolver based on smoothed aggregation (GES-SA) for initializing smoothed aggregation (SA) multigrid', Numer. Linear Algebra Appl. 15(2-3), 249269.

W. Briggs, V. Henson and S. McCormick (2000), A Multigrid Tutorial: Second Edition, Society for Industrial and Applied Mathematics. 


\section{CONCLUDING REMARKS}

T. F. Chan and W. L. Wan (2000), 'Robust multigrid methods for nonsmooth coefficient elliptic linear systems', J. Comput. Appl. Math. 123(1-2), 323-352. Numerical analysis 2000, Vol. III. Linear algebra.

T. F. Chan, J. Xu and L. Zikatanov (1998), 'An agglomeration multigrid method for unstructured grids', Contemporary Mathematics 218, 67-81.

T. Chartier, R. Falgout, V. Henson, J. Jones, T. Manteuffel, S. McCormick, J. Ruge and P. Vassilevski (2003), 'Spectral AMGe ( $\rho$ AMGe)', SIAM J. Sci. Comput. 25(1), 1 26.

L. Chen (2011), 'Deriving the X-Z identity from auxiliary space method', Journal of Domain Decomposition Methods in Science and Engineering XIX pp. 309-316.

L. Chen, R. H. Nochetto and J. Xu (2012), 'Optimal multilevel methods for graded bisection grids', Numer. Math. 120(1), 1-34.

P. G. Ciarlet (2002), The finite element method for elliptic problems, Vol. 40 of Classics in Applied Mathematics, Society for Industrial and Applied Mathematics (SIAM), Philadelphia, PA. Reprint of the 1978 original [North-Holland, Amsterdam; MR0520174 (58 \#25001)].

A. J. Cleary, R. D. Falgout, V. E. Henson and J. E. Jones (1998), Coarse-grid selection for parallel algebraic multigrid, in Solving irregularly structured problems in parallel (Berkeley, CA, 1998), Vol. 1457 of Lecture Notes in Comput. Sci., Springer, Berlin, pp. 104-115.

R. Courant and D. Hilbert (1924), Methoden der Mathematischen physik: Vol. 1, Julius Springer.

P. D'Ambra and P. Vassilevski (2014), 'Adaptive AMG based on weighted matching for systems of elliptic PDEs arising from displacements and mixed methods'. Submitted.

P. D'Ambra and P. S. Vassilevski (2013), 'Adaptive AMG with coarsening based on compatible weighted matching', Comput. Vis. Sci. 16(2), 59-76.

H. De Sterck, R. D. Falgout, J. W. Nolting and U. M. Yang (2008), 'Distance-two interpolation for parallel algebraic multigrid', Numerical Linear Algebra with Applications 15(2-3), 115-139.

H. De Sterck, K. Miller, E. Treister and I. Yavneh (2011), 'Fast multilevel methods for Markov chains', Numer. Linear Algebra Appl. 18(6), 961-980.

P. M. de Zeeuw (1990), 'Matrix-dependent prolongations and restrictions in a blackbox multigrid solver', J. Comput. Appl. Math. 33(1), 1-27.

J. E. Dendy, Jr. (1982), 'Black box multigrid', J. Comput. Phys. 48(3), 366-386.

J. E. Dendy, Jr. (1983), 'Black box multigrid for nonsymmetric problems', Appl. Math. Comput. 13(3-4), 261-283.

R. Diestel (2010), Graph theory, Vol. 173 of Graduate Texts in Mathematics, fourth edn, Springer, Heidelberg.

C. R. Dohrmann, A. Klawonn and O. B. Widlund (2008), A family of energy minimizing coarse spaces for overlapping Schwarz preconditioners, in Domain decomposition methods in science and engineering XVII, Vol. 60 of Lect. Notes Comput. Sci. Eng., Springer, Berlin, pp. 247-254.

Y. Efendiev, J. Galvis and P. S. Vassilevski (2011), Spectral element agglomerate algebraic multigrid methods for elliptic problems with high contrast coefficients, in Domain Decomposition Methods in Science and Engineering XIX (Y. Huang, R. Kornhuber, O. Widlund and J. Xu, eds), Vol. 78 of Lecture Notes in Computational Science and Engineering, Springer, Berlin, pp. 407-414. 
Y. R. Efendiev, T. Y. Hou and X.-H. Wu (2000), 'Convergence of a nonconforming multiscale finite element method', SIAM Journal on Numerical Analysis 37(3), 888-910.

R. Falgout (2004), A note on the relationship between adaptive AMG and PCG, Tech. Rep., Lawrence Livermore National Laboratory.

R. D. Falgout and P. S. Vassilevski (2004), 'On generalizing the AMG framework', SIAM J. Numer. Anal. 42(4), 1669-1693. Also available as LLNL technical report UCRLJC-150807.

R. D. Falgout, P. S. Vasilevski and L. T. Zikatanov (2005), 'On two-grid convergence estimates', Numerical linear algebra with applications 12(5-6), 471 - 494.

K. Fan (1949), 'On a theorem of weyl concerning eigenvalues of linear transformations i', Proceedings of the National Academy of Sciences of the United States of America 35(11), 652.

R. P. Fedorenko (1961), 'A relaxation method of solution of elliptic difference equations', Ž. Vyčisl. Mat. i Mat. Fiz. 1, 922-927.

R. P. Fedorenko (1964), 'On the speed of convergence of an iteration process', Ž. Vyčisl. Mat. i Mat. Fiz. 4, 559-564.

A. Gibbons (1985), Algorithmic graph theory, Cambridge University Press, Cambridge.

G. H. Golub and Q. Ye (1999/00), 'Inexact preconditioned conjugate gradient method with inner-outer iteration', SIAM J. Sci. Comput. 21(4), 1305-1320 (electronic).

L. Grasedyck, L. Wang and J. Xu (2015), 'A nearly optimal multigrid method for general unstructured grids', Numerische Mathematik pp. 1-30.

T. Grauschopf, M. Griebel and H. Regler (1997), 'Additive multilevel preconditioners based on bilinear interpolation, matrix-dependent geometric coarsening and algebraic multigrid coarsening for second-order elliptic PDEs', Appl. Numer. Math. 23(1), 63-95. Multilevel methods (Oberwolfach, 1995).

M. Griebel and P. Oswald (1995), 'On the abstract theory of additive and multiplicative Schwarz algorithms', Numer. Math. 70(2), 163-180.

W. Hackbusch (1977), On the computation of approximate eigenvalues and eigenfunctions of elliptic operators by means of a multi-grid method,, Report 77-10, Universität zu Köln.

W. Hackbusch (1978), A fast iterative method for solving Poisson's equation in a general region, in Numerical treatment of differential equations (Proc. Conf., Math. Forschungsinst., Oberwolfach, 1976), Springer, Berlin, pp. 51-62. Lecture Notes in Math., Vol. 631. Longer version: Ein Iteratives Verfahren zur Schnellen Auflösung Elliptischer Randwertprobleme, Math. Inst., Universität zu Köln, Report 76-12 (November 1976).

W. Hackbusch (1985), Multigrid methods and applications, Vol. 4 of Springer Series in Computational Mathematics, Springer-Verlag, Berlin.

W. Hackbusch (1989), 'The frequency decomposition multi-grid method. I. Application to anisotropic equations', Numer. Math. 56(2-3), 229-245.

W. Hackbusch (1994), Iterative solution of large sparse systems of equations, Vol. 95 of Applied Mathematical Sciences, Springer-Verlag, New York.

P. R. Halmos (1974), Finite-dimensional vector spaces, second edn, Springer-Verlag, New York-Heidelberg. Undergraduate Texts in Mathematics.

V. E. Henson and P. S. Vassilevski (2001), 'Element-free AMGe: general algorithms for computing interpolation weights in AMG', SIAM J. Sci. Comput. 23(2), 629-650 (electronic). Copper Mountain Conference (2000). 


\section{CONCLUDING REMARKS}

V. E. Henson and U. M. Yang (2002), 'BoomerAMG: a parallel algebraic multigrid solver and preconditioner', Appl. Numer. Math. 41(1), 155-177. Developments and trends in iterative methods for large systems of equations-in memoriam Rüdiger Weiss (Lausanne, 2000).

T. Hou, X.-H. Wu and Z. Cai (1999), 'Convergence of a multiscale finite element method for elliptic problems with rapidly oscillating coefficients', Mathematics of Computation of the American Mathematical Society 68(227), 913-943.

X. Hu, P. S. Vassilevski and J. Xu (2013), 'Comparative convergence analysis of nonlinear amli-cycle multigrid', SIAM J. Num. Anal 51(2), 1349-1369.

J. Jones and P. Vassilevski (2001), 'AMGe based on element agglomeration', SIAM J. Sci. Comput. 23(1), 109-133 (electronic).

K. Kahl (2009), 'Adaptive algebraic multigrid for lattice qcd computations', Ph.D. thesis, Bergische Universit'at Wuppertal, Fachbereich Mathematik und Naturwissenschaften, Wuppertal, Germany.

G. Karypis and V. Kumar (1998), 'A fast and high quality multilevel scheme for partitioning irregular graphs', SIAM Journal on scientific Computing 20(1), 359-392.

H. Kim, J. Xu and L. Zikatanov (2003), 'A multigrid method based on graph matching for convection diffusion equations', Numerical linear algebra with applications.

H. Kim, J. Xu and L. Zikatanov (2004), 'Uniformly convergent multigrid methods for convection-diffusion problems without any constraint on coarse grids', Adv. Comput. Math. 20(4), 385-399.

T. V. Kolev and P. S. Vassilevski (2006), 'AMG by element agglomeration and constrained energy minimization interpolation', Numer. Linear Algebra Appl. 13(9), 771-788.

J. Kraus (2008), 'Algebraic multigrid based on computational molecules, 2: Linear elasticity problems', SIAM Journal on Scientific Computing 30(1), 505-524.

J. Kraus and J. Schicho (2006), 'Algebraic multigrid based on computational molecules, 1: Scalar elliptic problems', Computing 77(1), 57-75.

J. K. Kraus (2002), 'An algebraic preconditioning method for $M$-matrices: linear versus non-linear multilevel iteration', Numer. Linear Algebra Appl. 9(8), 599-618.

J. H. Lai and L. N. Olson (2011), 'Algebraic multigrid for high-order hierarchical H(curl) finite elements', SIAM J. Sci. Comput. 33(5), 2888-2902.

Y.-J. Lee, J. Wu, J. Xu and L. Zikatanov (2007), 'Robust subspace correction methods for nearly singular systems', Math. Models Methods Appl. Sci. 17(11), 1937-1963.

Y.-J. Lee, J. Wu, J. Xu and L. Zikatanov (2008), 'A sharp convergence estimate for the method of subspace corrections for singular systems of equations', Math. Comp. 77(262), 831-850.

K. Lipnikov, J. D. Moulton and D. Svyatskiy (2011), 'Adaptive strategies in the multilevel multiscale mimetic $\left(\mathrm{M}^{3}\right)$ method for two-phase flows in porous media', Multiscale Model. Simul. 9(3), 991-1016.

O. Livne (2004), 'Coarsening by compatible relaxation', Num. Lin. Alg. Appl. 11(2), 205227.

O. Livne and A. Brandt (2012), 'Lean algebraic multigrid (LAMG): fast graph Laplacian linear solver', SIAM Journal on Scientific Computing 34(4), 499-523.

I. Livshits (2008), 'One-dimentional algorithm for finding eigenbasis of the Schrödinger operator', SIAM Journal on Scientific Computing 30(1), 416-440.

M. Luby (1986), 'A simple parallel algorithm for the maximal independent set problem', SIAM J. Comput. 15(4), 1036-1053. 
S. MacLachlan and Y. Saad (2007), 'A greedy strategy for coarse-grid selection', SIAM J. Sci. Comput. 29, 1825-1853.

S. MacLachlan, T. Manteuffel and S. McCormick (2006), 'Adaptive reduction-based AMG', Numer. Linear Algebra Appl. 13(8), 599-620.

S. P. MacLachlan and L. N. Olson (2014), 'Theoretical bounds for algebraic multigrid performance: review and analysis', Numer. Linear Algebra Appl. 21(2), 194-220.

S. P. MacLachlan, J. D. Moulton and T. P. Chartier (2012), 'Robust and adaptive multigrid methods: comparing structured and algebraic approaches', Numer. Linear Algebra Appl. 19(2), 389-413.

J.-F. Maitre and F. Musy (1983), 'Méthodes multigrilles: opérateur associé et estimations du facteur de converence: le cas du V-cycle', C. R. Acad. Sci. Paris Sér. I Math. 296(12), 521-524.

J. Mandel (1988), 'Algebraic study of multigrid methods for symmetric, definite problems', Appl. Math. Comput. 25, 39-56.

J. Mandel, M. Brezina and P. Vaněk (1999), 'Energy optimization of algebraic multigrid bases', Computing 62(3), 205-228.

T. Manteuffel, S. McCormick, M. Park and J. Ruge (2010), 'Operator-based interpolation for bootstrap algebraic multigrid', Numerical Linear Algebra with Applications pp. n/a-n/a.

I. Marek (1991), Aggregation methods of computing stationary distributions of Markov processes, in Numerical Treatment of Eigenvalue Problems Vol. 5/Numerische Behandlung von Eigenwertaufgaben Band 5, Springer, pp. 155-169.

A. M. Matsokin and S. V. Nepomnyashchikh (1985), 'The Schwarz alternation method in a subspace', Izv. Vyssh. Uchebn. Zaved. Mat. (10), 61-66, 85.

S. Mccormick and J. Ruge (1989), 'Algebraic multigrid methods applied to problems in computational structural mechanics'.

S. F. McCormick (1982), 'An algebraic interpretation of multigrid methods', SIAM J. Numer. Anal. 19, 548-560.

S. F. McCormick (1984), 'Multigrid methods for variational problems: further results', SIAM J. Numer. Anal. 21(2), 255-263.

S. F. McCormick (1985), 'Multigrid methods for variational problems: general theory for the $V$-cycle', SIAM J. Numer. Anal. 22(4), 634-643.

S. F. McCormick and J. W. Ruge (1982), 'Multigrid methods for variational problems', SIAM J. Numer. Anal. 19(5), 924-929.

S. Míka and P. Vaněk (1992a), 'Acceleration of convergence of a two level algebraic algorithm by aggregation in smoothing process', Appl. Math. 37, 343-356.

S. Míka and P. Vaněk (1992b), 'A modification of the two-level algorithm with overcorrection', Appl. Math. 37, 13-28.

A. Nägel, R. D. Falgout and G. Wittum (2008), 'Filtering algebraic multigrid and adaptive strategies', Comput. Vis. Sci. 11(3), 159-167.

A. Napov and Y. Notay (2012), 'An algebraic multigrid method with guaranteed convergence rate', SIAM Journal on Scientific Computing 34(2), A1079 - A1109.

R. A. Nicolaides (1975), 'On multiple grid and related techniques for solving discrete elliptic systems', J. Computational Phys. 19(4), 418-431.

R. A. Nicolaides (1977), 'On the $l^{2}$ convergence of an algorithm for solving finite element equations', Math. Comp. 31(140), 892-906. 


\section{CONCLUDING REMARKS}

Y. Notay (2000), 'Flexible conjugate gradients', SIAM J. Sci. Comput. 22(4), 1444-1460 (electronic).

Y. Notay (2010), 'An aggregation-based algebraic multigrid method', Electronic transactions on numerical analysis 37, 123-146.

Y. Notay (2012), 'Aggregation-based algebraic multigrid for convection-diffusion equations', SIAM journal on scientific computing 34(4), A2288 - A2316.

Y. Notay and P. Vassilevski (2008a), 'Recursive krylov-based multigrid cycles', Numerical Linear Algebra with Applications 15(5), 473-487.

Y. Notay and P. S. Vassilevski (2008b), 'Recursive Krylov-based multigrid cycles', Numer. Linear Algebra Appl. 15(5), 473-487.

L. N. Olson, J. B. Schroder and R. S. Tuminaro (2011a), 'A general interpolation strategy for algebraic multigrid using energy minimization', SIAM J. Sci. Comput. 33(2), 966-991.

L. N. Olson, J. B. Schroder and R. S. Tuminaro (2011b), 'A general interpolation strategy for algebraic multigrid using energy minimization', SIAM journal on scientific computing 33(2), $966-991$.

P. Oswald (1999), 'On the robustness of the BPX-preconditioner with respect to jumps in the coefficients', Math. Comp. 68(226), 633-650.

M. Reed and B. Simon (1978), IV: Analysis of Operators, Vol. 4, Elsevier.

A. Reusken (1993), 'Multigrid with matrix-dependent transfer operators for a singular perturbation problem', Computing 50(3), 199-211.

J. Ruge (1985), 'Final report on amg02, report, gesellschaft fuer mathematik und datenverarbeitung, st. augustin', GMD, contract 5110, 022090.

J. Ruge (1986), 'Amg for problems of elasticity', Applied Mathematics and Computation 19(1), 293-309.

J. W. Ruge (1983), Algebraic multigrid (amg) for geodetic survey problems, in Prelimary Proc. Internat. Multigrid Conference, Fort Collins, CO.

J. W. Ruge and K. Stüben (1987), Algebraic multigrid (AMG), in Multigrid Methods (S. F. McCormick, ed.), Vol. 3 of Frontiers in Applied Mathematics, SIAM, Philadelphia, PA, pp. 73-130.

Y. Saad (2003a), Iterative methods for sparse linear systems, second edn, Society for Industrial and Applied Mathematics, Philadelphia, PA.

Y. Saad (2003b), Iterative methods for sparse linear systems, second edn, Society for Industrial and Applied Mathematics, Philadelphia, PA.

J. B. Schroder (2012), 'Smoothed aggregation solvers for anisotropic diffusion', Numer. Linear Algebra Appl. 19(2), 296-312.

B. F. Smith, P. E. Bjørstad and W. D. Gropp (1996), Domain decomposition, Cambridge University Press, Cambridge. Parallel multilevel methods for elliptic partial differential equations.

R. V. Southwell (1940), Relaxation Methods in Engineering Science. A treatise on approximate computation, Oxford Engineering Science Series, Oxford University Press, New York.

R. V. Southwell (1946), Relaxation Methods in Theoretical Physics, Oxford, at the Clarendon Press.

N. Spillane, V. Dolean, P. Hauret, F. Nataf, C. Pechstein and R. Scheichl (2014), 'Abstract robust coarse spaces for systems of PDEs via generalized eigenproblems in the overlaps', Numer. Math. 126(4), 741-770. 
H. Sterck, U. Yang and J. Heys (2006), 'Reducing complexity in parallel algebraic multigrid preconditioners', SIAM J. Matrix Anal. Appl. 27(4), 1019-1039.

K. Stüben (1983), 'Algebraic multigrid (AMG): experiences and comparisons', Applied Mathematics and Computation 13(3-4), 419-451.

A. Toselli and O. Widlund (2005), Domain decomposition methods-algorithms and theory, Vol. 34 of Springer Series in Computational Mathematics, Springer-Verlag, Berlin.

E. Treister and I. Yavneh (2015), 'Non-Galerkin multigrid based on sparsified smoothed aggregation', SIAM J. Sci. Comput. 37(1), A30-A54.

U. Trottenberg, C. W. Oosterlee and A. Schüller (2001), Multigrid, Academic Press, Inc., San Diego, CA. With contributions by A. Brandt, P. Oswald and K. Stüben.

I. Vakhutinsky, L. Dudkin and A. Ryvkin (1979), 'Iterative aggregation-A new approach to the solution of large-scale problems', Econometrica: Journal of the Econometric Society pp. 821-841.

P. Vaněk, J. Mandel and M. Brezina (1996a), 'Algebraic multigrid based on smoothed aggregation for second and fourth order problems', Computing 56, 179-196.

P. Vaněk, J. Mandel and M. Brezina (1996b), 'Algebraic multigrid by smoothed aggregation for second and fourth order elliptic problems', Computing 56(3), 179-196. International GAMM-Workshop on Multi-level Methods (Meisdorf, 1994).

P. Vaněk, J. Mandel and M. Brezina (1998), 'Convergence of algebraic multigrid based on smoothed aggregation', Computing 56, 179-196.

R. S. Varga (2000), Matrix iterative analysis, Vol. 27 of Springer Series in Computational Mathematics, expanded edn, Springer-Verlag, Berlin.

P. S. Vassilevski (2008a), Multilevel block factorization preconditioners, Springer, New York. Matrix-based analysis and algorithms for solving finite element equations.

P. S. Vassilevski (2008b), Multilevel block factorization preconditioners, Springer, New York.

P. S. Vassilevski and L. T. Zikatanov (2006), 'Multiple vector preserving interpolation mappings in algebraic multigrid', SIAM J. Matrix Anal. Appl. 27(4), 1040-1055 (electronic).

C. Wagner and G. Wittum (1997), 'Adaptive filtering', Numer. Math. 78(2), 305-328.

W. L. Wan (1998), Scalable and multilevel iterative methods, PhD thesis, UNIVERSITY OF CALIFORNIA Los Angeles.

W. L. Wan, T. F. Chan and B. Smith (1999/00), 'An energy-minimizing interpolation for robust multigrid methods', SIAM J. Sci. Comput. 21(4), 1632-1649.

W. Weiler and G. Wittum (1997), 'Parallel frequency filtering', Computing 58(4), 303316.

H. Weyl (1911), 'Über die asymptotische verteilung der eigenwerte', Nachrichten von der Gesellschaft der Wissenschaften zu Göttingen, Mathematisch-Physikalische Klasse 1911, 110-117.

H. Weyl (1912), 'Das asymptotische verteilungsgesetz der eigenwerte linearer partieller differentialgleichungen', Math. Ann 71, 441-479.

O. B. Widlund (1994), Exotic coarse spaces for Schwarz methods for lower order and spectral finite elements, in Domain decomposition methods in scientific and engineering computing (University Park, PA, 1993), Vol. 180 of Contemp. Math., Amer. Math. Soc., Providence, RI, pp. 131-136. 


\section{CONCLUDING REMARKS}

O. B. Widlund (2009), The development of coarse spaces for domain decomposition algorithms, in Domain decomposition methods in science and engineering XVIII, Vol. 70 of Lect. Notes Comput. Sci. Eng., Springer, Berlin, pp. 241-248.

G. Wittum (1992), Filternde Zerlegungen, Teubner Skripten zur Numerik. [Teubner Scripts on Numerical Mathematics], B. G. Teubner, Stuttgart. Schnelle Löser für große Gleichungssysteme. [Fast solvers for large systems of equations].

J. Xu (1989), Theory of multilevel methods, Vol. 8924558, Cornell University, May.

J. Xu (1991), 'Counterexamples concerning a weighted $L^{2}$ projection', Math. Comp. 57(196), 563-568.

J. Xu (1992), 'Iterative methods by space decomposition and subspace correction', SIAM Rev. 34(4), 581-613.

J. Xu (1996), 'The auxiliary space method and optimal multigrid preconditioning techniques for unstructured grids', Computing 56(3), 215-235.

J. Xu (1997), An introduction to multilevel methods, in Wavelets, multilevel methods and elliptic PDEs (Leicester, 1996), Numer. Math. Sci. Comput., Oxford Univ. Press, New York, pp. 213-302.

J. Xu (2009), Optimal algorithms for discretized partial differential equations, in ICIAM 07-6th International Congress on Industrial and Applied Mathematics, Eur. Math. Soc., Zürich, pp. 409-444.

J. Xu (2016), Multilevel iterative methods, Lecture Notes 1989-2016.

J. Xu and L. Zikatanov (2002), 'The method of alternating projections and the method of subspace corrections in Hilbert space', J. Amer. Math. Soc. 15(3), 573-597.

J. Xu and L. Zikatanov (2004), 'On an energy minimizing basis for algebraic multigrid methods', Comput. Vis. Sci. 7(3-4), 121-127.

J. Xu and J. Zou (1998), 'Some nonoverlapping domain decomposition methods', SIAM review 40(4), 857-914.

J. Xu, H. Zhang and L. Zikatanov (2016a), 'Obtaining optimal coarse spaces for AMG via trace minimization'.

J. Xu, H. Zhang and L. Zikatanov (2016b), 'On the Weyl's law for discretized elliptic operators'.

J. Xu, H. Zhang and L. Zikatanov (2016c), 'A unified approach to the design and analysis of AMG'.

U. M. Yang (2006), Parallel algebraic multigrid methods-high performance preconditioners, Springer.

D. M. Young (1971), Iterative solution of large linear systems, Academic Press, New York-London.

H. Yserentant (1993), Old and new convergence proofs for multigrid methods, in Acta numerica, 1993, Acta Numer., Cambridge Univ. Press, Cambridge, pp. 285-326.

G. Yu, J. Xu and L. T. Zikatanov (2013), 'Analysis of a two-level method for anisotropic diffusion equations on aligned and nonaligned grids', Numerical Linear Algebra with Applications 20(5), 832-851.

L. Zikatanov (2008), 'Two-sided bounds on the convergence rate of two-level methods', Numerical Linear Algebra with Applications 15(5), 439-454. 